NASA CONTRACTOR REP O R T

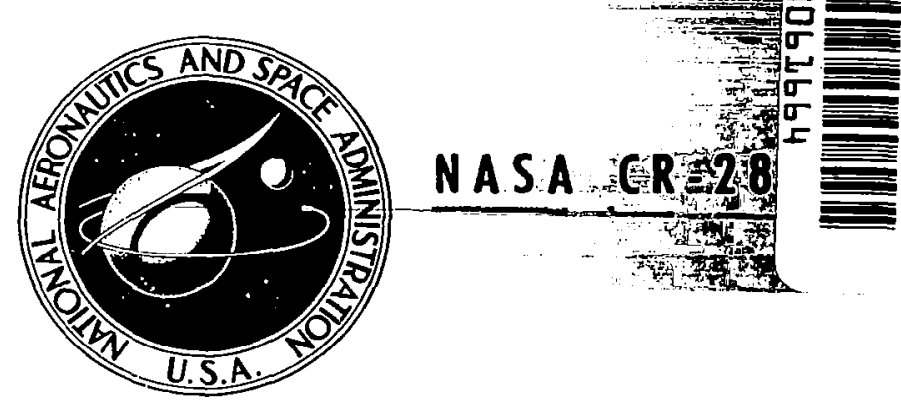

LOAN COPY: RETURN TO AFWL TECHNICAL LIBRAF KIRTLAND AFB, N. M.

\title{
VORTEX INTERACTIONS AND DECAY IN AIRCRAFT WAKES
}

Alan J. Bilanin, Milton E. Teske,

Coleman duP. Donaldson, and Guy G. Williamson

Prepared by

AERONAUTICAL RESEARCH ASSOCIATES OF PRINCETON, INC.

Princeton, N.J. 08540

for Langley Research Center

National aERONAUtics and SPACE ADMINISTRATION - WASHINGTON, D. C. - SEPTEMBER 1977 


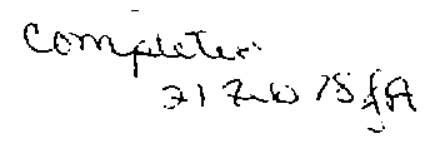

\section{ERRATA}

NASA Contractor Report 2870

VORTEX INTERACTIONS AND DECAY IN AIRCRAFT WAKES
Alan J. Bilanin, Milton E. Teske, Coleman duP. Donaldson, and Guy G. Williamson
September 1977

Block 11 on the Technical Report Standard Title Page (Abstract page) is in error. The contract number should be NAS1-13932. A corrected page is attached.

Issued January 1978 


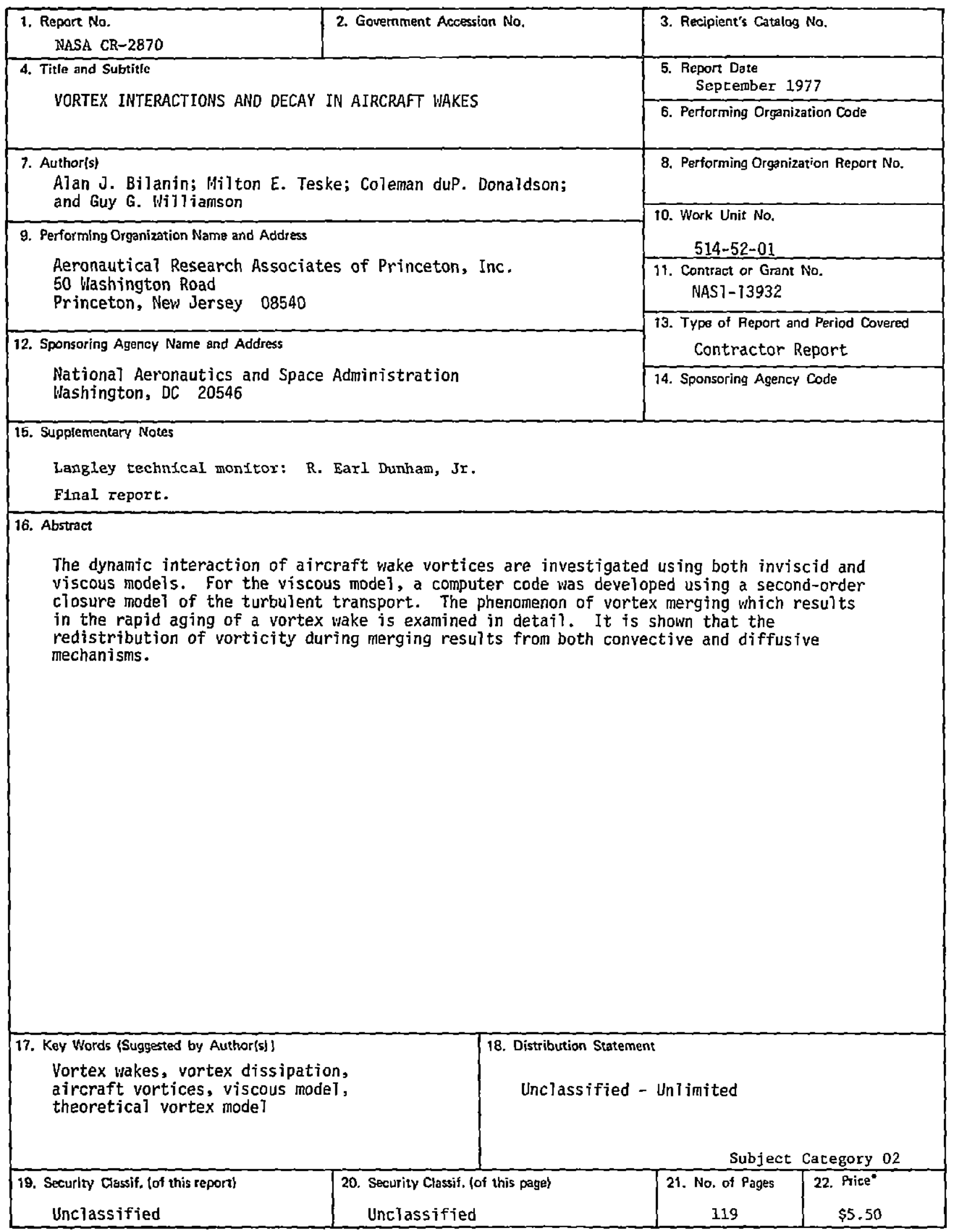

'For sale by the National Technical Information Service, Springfield, Virginia 22151 
1. Report No.

NASA $C R-2870$

4. Title and Subtitle

VORTEX INTERACTIONS AND DECAY IN AIRCRAFT WAKES
2. Government Accession No.

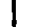

No.

3. Recipient's Catalog No.

5. Report Date

September 1977

6. Performing Organization Code

8. Performing Organization Report No.

7. Author(s)

Alan J. Bilanin; Milton E. Teske; Coleman duP. Donaldson;

and Guy G. Wi T liamson

9. Performing Organization Name and Address

Aeronautical Research Associates of Princeton, Inc. 50 Washington Road

Princeton, New Jerscy 08540

12. Sponsoring Agency Name and Address

National Aeronautics and Space Administration Washington, DC 20546

10. Work Unit No.

514-52-01

11. Contract or Grant No.

NAS1-1393:

13. Type of Report and Period Covered

Contractor Report

14. Sponsoring Agency Code

15. Supplementary Notes

Langley technical monitor: R. Earl Dunham, Jr.

Final report.

16. Abstract

The dynamic interaction of aircraft wake vortices are investigated using both inviscid and viscous models. For the viscous model, a computer code was developed using a second-order closure model of the turbulent transport. The phenomenon of vortex merging which results in the rapid aging of a vortex wake is examined in detail. It is shown that the redistribution of vorticity during merging results from both convective and diffusive - mechanisms.

17. Key Words (Suggested by Author(s))

Vortex wakes, vortex dissipation, aircraft vortices, viscous model, theoretical vortex model
18. Distribution Statement

Unclassified - Unlimited

Subject Category 02

19. Security Classif. (of this report)

Unclassified

20. Security Classif. (of this pagel Unclassified
21. No. of Pages

119
22. Price*

$\$ \$ 5.50$

"For sale by the National Technical Information Service, Springfield, Virginia 22151 
VORTEX INTERACTIONS AND DECAY IN AIRCRAFT WAKES

\author{
Alan J. Bilanin, Milton E. Teske, Coleman duP. Donaldson, \\ and Guy G. Williamson \\ Aeronautical Research Associates of Princeton, Inc. \\ Princeton, New Jersey
}

\title{
SUMMARY
}

The dynamic interactions of aircraft wake vortices are investigated using both inviscid and viscous models. The phenomenon of vortex merging which results in the rapid aging of a vortex wake is examined in detail. It is shown that the redistribution of vorticity during merging results from both convective and diffusive mechanisms.

Inviscid computations show that the merging phenomenon is sensitive to small changes in the spanwise load distribution. In particular, the fuselage vortex, shed from the wing-fuselage junction, can play a significant role in bringing about merging of wing tip and flap vortices. The usefulness of point vortex computations in predicting merging is also explored.

A vortex wake code including turbulent transport is developed using a second-order closure model of the turbulent transport. Vortex wake merging computations using this code indicate that a low hazard wake occurs when the generating aircraft trails flap and wing tip vortices from each side of the wing of the same strength and sign. To achieve this optimum, the flap vortex is located outboard at approximately $40 \%$ of the distance to the tip vortex.

Preliminary computations of multivortex wakes interacting with the ground suggest that the merging phenomenon may be altered by the presence of this ground plane. Computations have shown that jet exhaust does not alter the merging phenomenon. The effect of ambient atmospheric turbulence on the aging of an aircraft wake is investigated at constant turbulent dissipation rate. It is shown that under stable atmospheric conditions, when atmospheric macroscales are less than or equal to the vortex spacing, misleading results may be obtained. 


\section{INTRODUCTION}

Low hazard aircraft vortex wakes are produced when the configuration of an aircraft is such that multiple vortices are trailed which interact strongly enough to result in merging. The mechanism of merging involves the convective and turbulent redistribution of trailed vorticity. The merging phenomenon in aircraft vortex wakes or the pairing, condensing, or coalescing phenomenon in turbulent shear layers is decidedly nonlinear. Thus, an experimental or numerical approach to the study of the phenomenon has been favored. There is now a need to understand it more fully. In particular, a model which could predict the redistribution of vorticity during merging would aid us in our search for an aircraft configuration of low vortex hazard. The work reported here reviews the methods that are available to investigate aircraft vortex wakes and describes a new technique which has been developed to give a detailed description of the viscous interactions in a wake. This new technique makes use of a code which solves the fluid equations of motion including turbulent transport through a second-order turbulent closure of the Reynolds stress equations.

It is appropriate to introduce the merging concept here in order that the reader may initially see the significance of the phenomenon. Consider the wakes of an aircraft in the cruise configuration and in the landing configuration as schematically illustrated in figure 1 . The trailed vorticity of the cruise wake is found in two regions and the distributions are nearly axisymmetric. Consequently, the production of turbulence and the subsequent diffusion of vorticity across the nearly axisymmetric streamlines are suppressed by the centrifugal effect of the swirling velocity. The aging in the absence of atmospheric turbulence, wind shear, or stratification results only from the modest amounts of turbulence generated in the vortices.

When an aircraft is in a landing or takeoff configuration, multiple vortices are trailed to form a wake. These vortices are both convected and strained by each other. This induced straining field is responsible for the merging or pairing of the two vortices, which then results in the production of turbulence and even wider redistribution of the trailed vorticity. We have tried schematically to illustrate in figure 1 the fact that at comparable times after aircraft passage the multiple-vortex wake has a more widely spread distribution of vorticity than does the wake from an aircraft in the cruise configuration. It should be apparent that if circulation in the half-plane is taken as a measure of the strength of the wake, no reduction in the circulation can occur until the trailed vorticity has been diffused to the wake centerline. The turbulence produced during merging provides the mechanism for this diffusion. 
II positive vorticity

MI negative vorticity

$t=0$

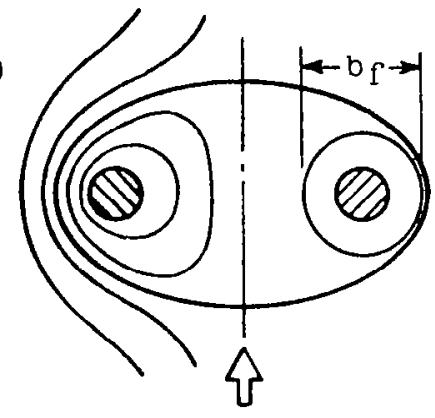

Single vortex pair

(In all figures, a uniform upward velocity is assumed to cancel wake descent) $t=0$

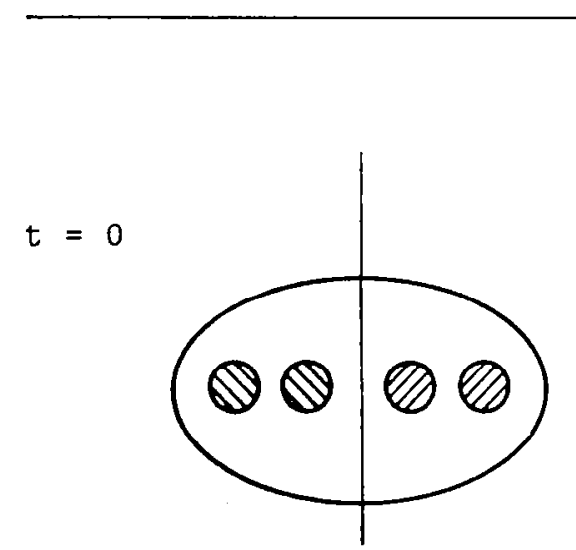

Multiple-pair vortex wake

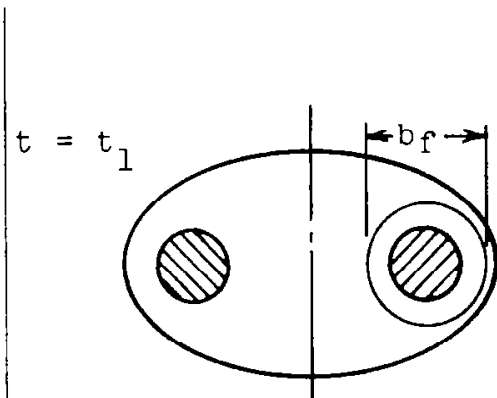

Trailed vorticity slowly diffused by viscous transport diffused by viscous transport

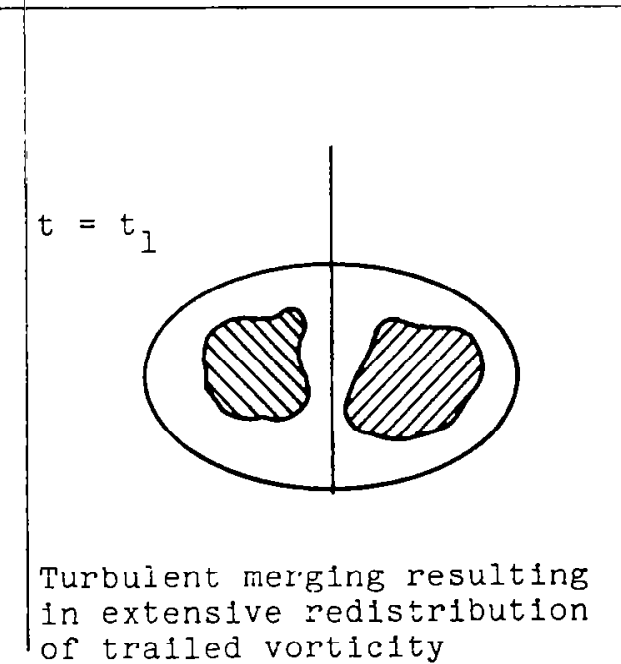

$t=t_{2}$

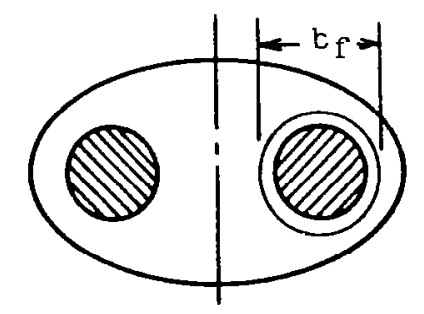

Trailed vorticity still not

extensively diffused throughout wake

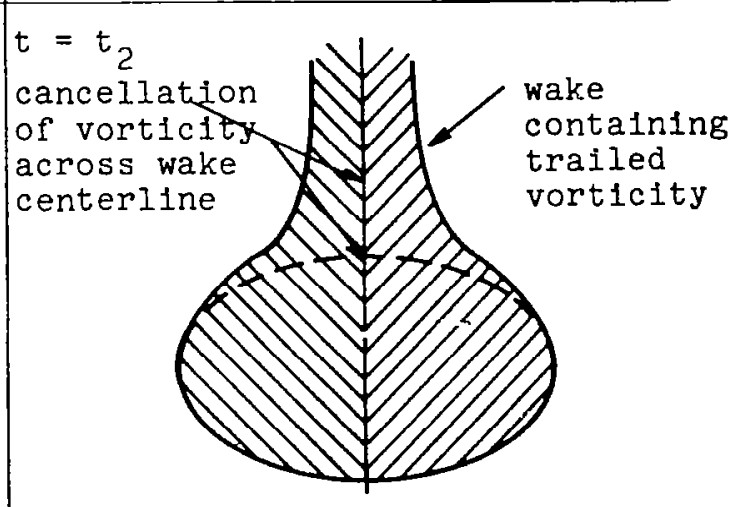

Aged wake; vorticity is shed in a wake and also diffuses across wake centerline

Figure 1. An idealization of the processes which result in the aging of a single- and multiple-pair vortex wake. The encountering aircraft's span is denoted by $b_{f}$. 
The viscous interactions in an aircraft wake cannot be ignored if there is to be any attempt to predict the decay of wake intensity with distance downstream of the aircraft. While this report treats both inviscid and viscous interactions, our emphasis is on transport phenomena. The report is organized in five parts; the first (Section 2) reviews inviscid techniques to compute wake structure and vortex-vortex interaction. The mechanism responsible for merging is discussed and an inviscid study of the vortex wake of a B-747 aircraft is described. In Section 3 a description of our turbulent model based on a second-order closure of the Reynolds stress equations is given. A description of the computer code which solves the turbulent model is given in Section 4, and a variety of vortex wake interaction problems are posed and solved in section 5. Finally, in section 6, conclusions and recommendations are offered.

\section{NOMENCLATURE}

A

$\mathrm{b}$

$\mathrm{b}_{\mathrm{T}}$

$c$

$c_{\ell}$

$\bar{c}_{p}^{c}$

$\bar{c}_{\mathrm{w}}$

$\mathrm{C}_{\ell}$

$\mathrm{C}_{\mathrm{L}}$

$\mathrm{C}_{\mathrm{m}_{\mathrm{O}}}$

d

g

h

L

$\mathrm{M}_{\mathbf{i}}$

$P$

q

$\overline{\mathrm{r}}$

$r_{c}$ aspect ratio and turbulent model constant

wing span and turbulent model constant

tail span

local chord

sectional lift coefficient

center of pressure

wing reference chord

rolling moment coefficient

lift coefficient

moment coefficient at $C_{L}=0$

length

gravity

height of tail above initial wing wake vortex sheet; height above ground

lift

angular momentum in fluid in bounded region due to ith vortex

pressure

root mean square of twice the turbulent kinetic energy

position vector

vorticity spread length scale 
$S$

$s, s_{1}, s_{2}, s_{3}, s_{4}$

$S$

$t$

$\mathrm{U}, \mathrm{V}, \mathrm{W}$

$\mathrm{U}_{\infty}$

$\mathrm{V}$

$\mathrm{x}, \mathrm{y}, \mathrm{z}$

$\overline{\mathrm{y}}$

$\alpha$

$\Gamma$

$\delta_{f_{i}}$

$\delta_{f_{m}}$

$\delta_{\text {LEF }}$

$\varepsilon$

$\zeta$

$\theta$

$\theta$

$\lambda$

$\Lambda$

$v$

$\rho$

$\psi$

Subscripts:

$\mathrm{f}$

g

$t, T$

W wing semi-span

turbulent model constants

reference area

time

velocity components in a Cartesian $x, y, z$ system

free stream velocity

model diffusion coefflcient

Cartesian coordinates

centroid of trailed vorticity

angle between local section of wing and reference Iine when subscripted or standard angle of attack when not subscripted

circulation

inboard trailing edge flap

midspan trailing edge flap

leading edge flap angle

turbulent dissipation rate

axial, streamwise, or $x$ component of vorticity

fluctuating temperature

mean temperature

turbulent dissipation scale

macroscale or integral scale parameter

kinematic viscosity

density

stream function

follower or flap

generator

tail

wing 


\section{INVISCID DESCRIPTION OF AIRCRAFT WAKES}

While a mathematical model of an aircraft wake must include turbulent transport if there is to be any attempt to predict aging of the wake, considerable insight into wake structure and dynamics can be obtained from an inviscid description of the wake. Inviscid techniques which are used to predict wake geometry and structure are reviewed here. Emphasis is placed on limitations of the models in an effort to make us cognizant of their range of applicability. The technique of using point vortices to approximate the rollup and subsequent vortex interactions of an aircraft vortex wake receives special emphasis, and calculations are undertaken to systematically investigate the B-747 at a lift coefficient of 1.2. What will become clear is that favorable vortex wake interactions are quite sensitive to small changes in wing load distribution. In particular, inviscid analyses show that the strength and position of the fuselage vortex, which is trailed as a result of lift dropoff at the wing-fuselage junction, play a subtle roll in the favorable merging phenomenon. This result and other results which are obtained here bring out the need for measured wing load distribution data.

\subsection{Method of Betz and Extensions}

The method of Betz (ref. 1) relates the load distribution on a simply loaded wing to two axisymmetric distributions of swirling velocity in the distant rolled-up wake. The method is based on the application of certain "exact" integral invariants of a two-dimensional distribution of vorticity in a physically motivated manner. Many investigators have re-examined the Betz procedure and have extended the technique to include multiple vortices as well as distributed wing drag. These extensions have been recently reviewed in reference 2 .

The comparison of measured swirl velocity distributions in the wakes of clean aircraft (cruise conflguration) with distributions predicted by the Betz technique is remarkably good (ref. 3). However, when an aircraft is in a dirty (landing or takeoff) configuration, the Betz technique has predicted velocity distributions which depart significantly from measured distributions (ref. 4). This discrepancy has been explained by the observations first made by Dunham (ref. 5) of the strong interaction and coalescing or merging of multiple vortices into a single, more diffuse vortex. The Betz method assumes a priori that each vortex trailed in the wake rolls up independently. That is to say that other vortices in the wake do not affect vortex roll-up or structure. The merging phenomenon is one in which the straining of vortex on vortex is the dominant interaction. Therefore, the prediction by the Betz technique of the vortex velocity distributions in a multiple-vortex-pair wake, where vortices (particularly vortices of like sign, as we will 
show) come in close proximity, must necessarily fail. The Betz technique has proven to be a simple means of obtaining a first look at the structure of aircraft vortex wakes. It cannot, however, be used in situations where straining interactions between vortices are important. The technique is still quite useful as a means of specifying approximate initial velocity conditions for the numerical wake code described in Section 4.

\subsection{Inviscid Description of Merging}

A physical description of the merging phenomenon can be obtained from a simple model computation first undertaken by Corsiglia, et al. (ref. 6). If a weak vortex of vanishingly small strength 1 s placed in the velocity field of a second vortex of strength $\Gamma$, the weak vortex is simply convected about the strong vortex. The structure of the strong vortex is unimportant since it cannot change in this inviscid calculation. The weak vortex has a circular region of diameter d containing negligible vorticity and is initially positioned at $\mathrm{z} / \mathrm{d}=1.0$, as shown in figure 2 . The strong vortex is positioned at $y=z=0$. As the computation proceeds, the weak distribution is strained about the strong vortex and, as shown, becomes progressively more and more wrapped about it. It is significant that the initially circular region becomes a spiralled sheet and that the fluid between the spiralled sheet is irrotational. Of course, transport will quickly act to smooth this vorticity distribution so that the end state is one nearly axisymmetric vortex with vorticity distributed over a circular area of diameter greater than $2 \mathrm{~d}$. When the merging phenomenon occurs between vortices of nearly the same strength, the axial symmetries of both vortices are destroyed by the strain of vortex upon vortex. While the initial phases of merging simply involve convection, turbulent transport soon becomes the mechanism by which vorticity is redistributed outward. When present in aircraft wakes, merging results in a significant reduction of the rolling moment induced on an encountering aircraft. The outward redistribution of the trailed vorticity, which promotes the rapid aging of the wake, is the mechanism responsible for the reduction in rolling moment.

Since the initial phase of merging is a convective redistribution of vorticity, attempts have been made to predict analytically and numerically the strain field which will not allow a vortex to remain an entity. Moore and Saffman (ref. 8) have given an analysis of a vortex of elliptic cross-section in which the vorticity is uniform. This vortex is embedded in a flow field composed of a pure strain combined with a far field of solid body rotation; that is, the flow field induced by one vortex on another. It has been possible to apply their analysis to determine the proximity $d$ and strength $\Gamma_{2}$ at which a vortex of initial diameter $d_{c}$ and strength $\Gamma_{1}$ (uniform vorticity) will not yield a steady vortex solution of elliptical cross-section. Unfortunately, this does not imply that merging is imminent, since unsteady 


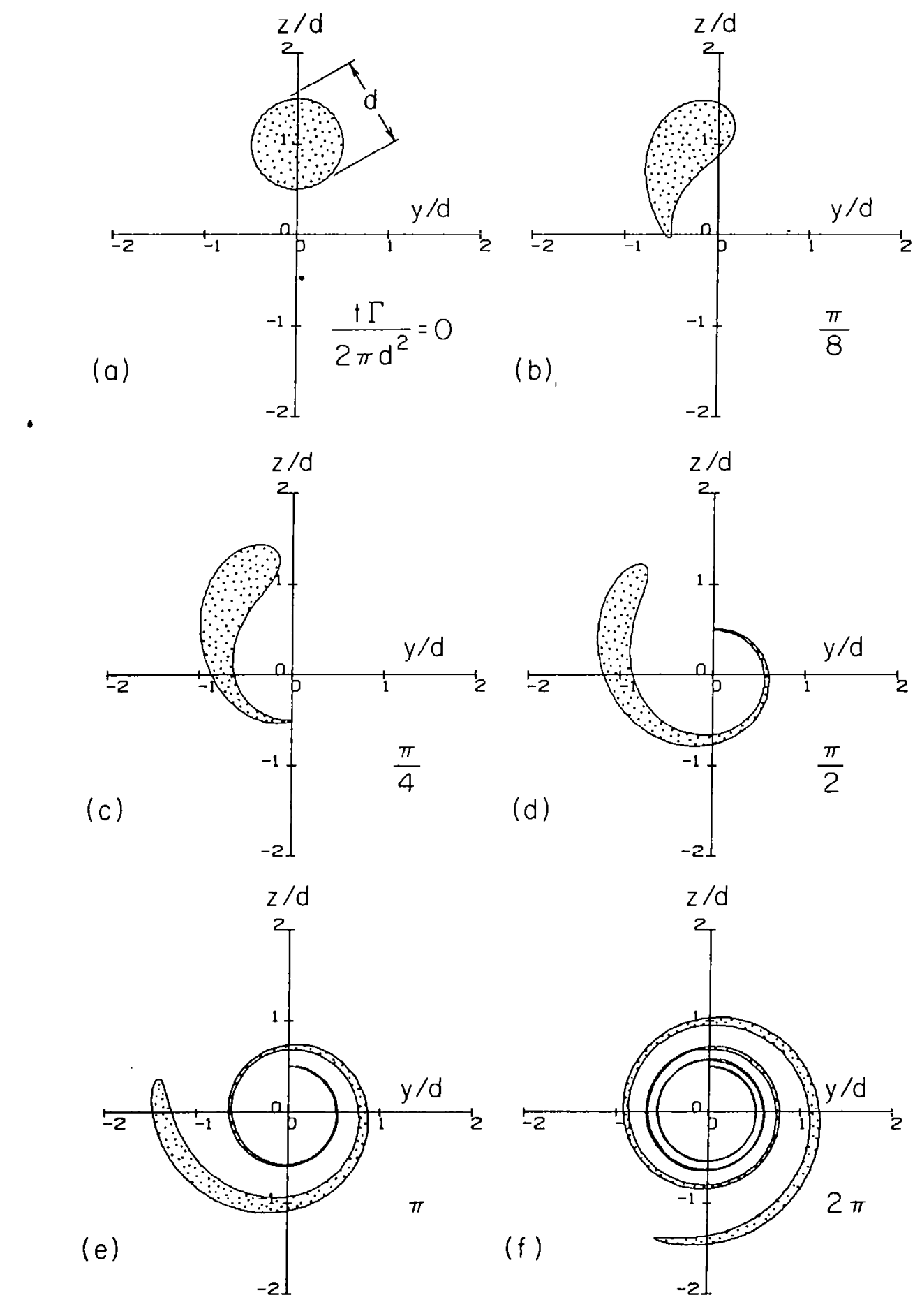

Figure 2. The inviscid merging of a vortex of vanishingly small strength with a very strong vortex 
solutions may still exist. However, as we will show, numerical experiments using large numbers of point vortices to represent the distributions of vorticity suggest that the criterion obtained is a good estimate of the strain required to bring about merging. The details of this computation may be found in Appendix $A$.

In figure 3 are shown the curves of critical relative vortex strength versus vortex separation $d / d_{c}$ below which steady stable solutions do not exist. Also shown is the merging boundary determined by Rossow (ref. 9) in his investigation of the merging phenomenon between two vortices of uniform vorticity having the same core diameter $d_{c}$. Typically, 40 point vortices are used to represent each vortex distribution. Also shown is the critical separation ratio for $\Gamma_{2} / \Gamma_{1}=1.0$ as computed by Roberts and Christiansen (ref. 10). Here it is estimated that several hundred point vortices have been used to represent each distribution. The agreement is reasonable. We point out, that in any case the analytic model is not exactly the fluid flow being computed by the distributions of point vortices. That is because the vortex in the analytic model which is providing the straining field is not permitted to have any structure--it is a point vortex of strength

$\Gamma_{2}$. It is, however, heartening to find the favorable comparison.

Judging simply by the areas in which merging is predicted, it is suggested quite strongly that merging between vortices of like sign is more probable than merging between vortices of unlike sign. This observation has recently been confirmed in a wind tunnel using wings to generate tip vortices which are marked with smoke (ref. 7 ).

2.3. Wake Rol1-Up and Vortex Interactions Using Point Vortices

Point vortex computations have a widespread popularity among researchers and have been used to model shear layers as well as aircraft vortex wakes. The object of this section is twofold:

a) to compute some measure of the roll torque experienced by a follower aircraft in the wake of a B-747 aircraft in trim configuration modeled by a distribution of point vortices;

b) to present a critical discussion in an attempt to define the practical limits of applicability of point vortex computations. Results obtained by the point vortex technique are compared with experimental data where possible. The procedure used to estimate the angular momentum which can result in a roll torque on a following aircraft that encounters the wake is depicted in figure 4 and is outlined below. 


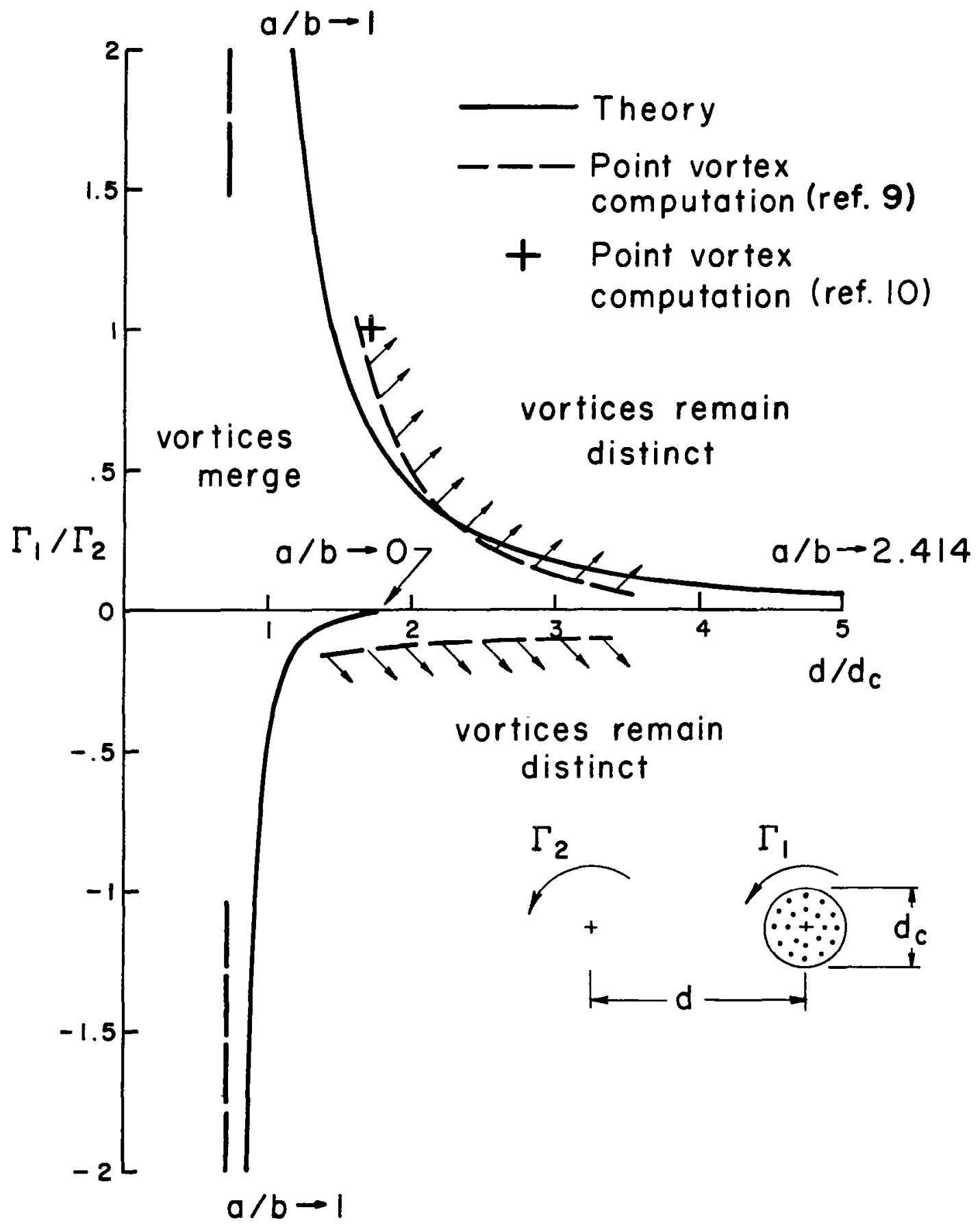

F1gure 3. Merging boundaries for a vortex with a uniform vorticity core as computed from the model given in reference 8 
Basic inputs are:

1) Points specifying wing planform with no leading or trailing edge flap deflections

2) Leading and trailing edge flap deflections

3) Total lift coefficient

4) Wing-tail areas and longitudinal positions relative to aircraft c.g.

5) Desired number of chordwise and spanwise subdivisions of wing for vortex lattice model

Calculate spanwise lift distribution, angle of attack, and tail lift coefficient.

Desired maximum number
of line vortices in
wake model

Calculate strength and initial positions of wing and tail vortices.

\begin{tabular}{|c|}
\hline $\begin{array}{l}\text { Calculate vortex trajectories as they convec- } \\
\text { tively interact with one another. }\end{array}$ \\
\hline $\begin{array}{l}\text { Using a hazard criterion such as angular } \\
\text { momentum of the fluid in a bounded region, } \\
\text { survey the wake and evaluate its hazard } \\
\text { potential. }\end{array}$ \\
\hline
\end{tabular}

Figure 4. Flow chart for wake hazard evaluation using discrete inviscid vortices 
In Appendix B the procedures used to compute the discrete vortex wake behind a generating aircraft is given. With the positions of discrete vortices given, the remaining step is to evaluate how hazardous the flow field is for smaller aircraft.

Hazard criterion for vortex wakes modeled by distributions of point vortices.- Using strip theory, the rolling moment induced by a point vortex located in the plane of a following wing of semispan $\mathrm{s}$ is proportional to

$$
c_{\ell} \propto \int_{-S}^{s} \frac{y}{y-d} d y
$$

where the vortex is located at $\mathrm{y}=\mathrm{d}$ and the airfoil is assumed to have constant chord and constant sectional lift coefficient. When $d>s$, the Integral is well behaved; however, when $-\mathrm{s}<\mathrm{d}<$ $\mathrm{s}$, the integral must be interpreted as a Cauchy principal value. The difficulty, of course, occurs when $d= \pm s$. Here the rolling moment is undefined. This singularity has made it difficult in the past to interpret results of point vortex computations quantitatively.

The angular momentum in a circle of radius $s$ is a well defined quantity which is related to the torque induced on an encountering wing. Physically the magnitude of the angular momentum in the fluid immediately surrounding the following aircraft must give a measure of the induced rolling moment. Exception must be made for certain pathological situations which do not appear relevant to the discussion here.

The angular momentum of the fluid in a circle of radius $s$ due to a single point vortex as shown in the following sketch is a straightforward computation. The coordinate system is centered on the vortex center with $y$ axis through the center of the circular window located at $\bar{r}_{c}$. The angular momentum with respect to the center of the circular window due to the ith vortex is

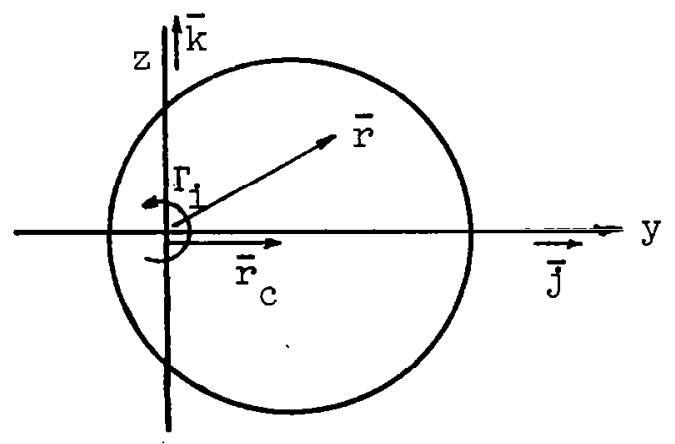




$$
\mathrm{M}_{\mathcal{L}}=\int\left(\overline{\mathrm{r}}-\overline{\mathrm{r}}_{\mathrm{c}}\right) \times \overline{\mathrm{v}} \mathrm{dA}
$$

Substituting for each of the vectors

$$
\begin{aligned}
\bar{r} & =y \bar{j}+z \bar{k} \\
\bar{r}_{c} & =d_{1} \bar{j} \\
\bar{v} & =\frac{r_{1}}{2 \pi\left(y^{2}+z^{2}\right)}(z \bar{j}-y \bar{k})
\end{aligned}
$$

and performing the integration yields

$$
M_{i}=\frac{\Gamma_{i}}{2}\left(s^{2}-a_{i}^{2}\right)
$$

It can be shown that vortices which are outside of the circular window do not contribute to the angular momentum. The contribution due to each of the vortices in the wake model is calculated and summed to find the total angular momentum of the fluid within the circle. The computation is repeated with the circle moved laterally and vertically until the wake field at a particular downstream station has been completely surveyed and the maximum value of angular momentum found. This value is then used as a hazard measure for different wakes with different size follower aircraft.

The techniques described above have been implemented in a code and are now used to study the wake flow behind a B-747 aircraft.

Results of inviscid point vortex computation.- The inviscid point vortex wake model was used to study theoretically the wake flow field generated by a B-747 aircraft at a lift coefficient of 1.2. First the wing load distributions were computed for a matrix of flap settings. The c.g. position of the aircraft was assumed to be $29 \%$ of the mean aerodynamic chord, and the aircraft was in trim with leading edge flaps fully extended--typical for landing. The following matrix of flap angle settings was investigated and is shown with the computed wing and tail coefficients. 


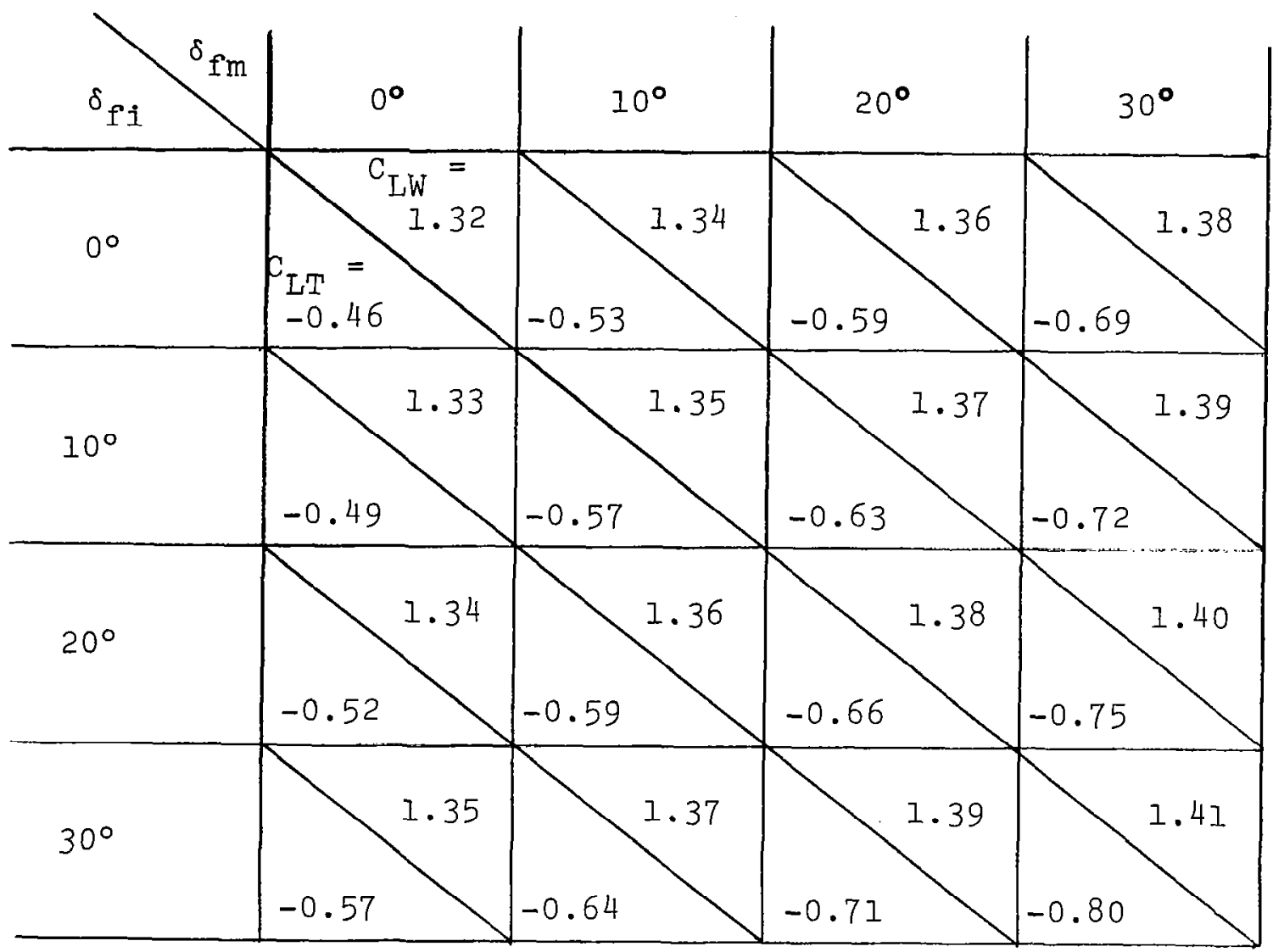

A spanwise lift distribution for each corner of the matrix, as determined by the vortex lattice program, is shown in figure 5 . At this point, we note what will be shown in the next subsection to be one limitition of the computed results presented here. The presence of the fuselage and the associated lift drop-off at the wing-fuselage junction cannot be calculated with this vortex lattice code.

Each load distribution was discretized with approximately 40 pairs of line vortices plus one pair for the tail vortex, and trajectories were computed to a downstream distance of about 1.5 $\mathrm{km}$. Integration step size had to be a compromise between satisfying a courant condition and avolding accumulation of roundoff error. In any event, a compromise was struck, and calculations all proceeded with the integral invariants of impulse and difference kinetic energy (see ref. 2) not varying by more than a few percent from their initial values. 


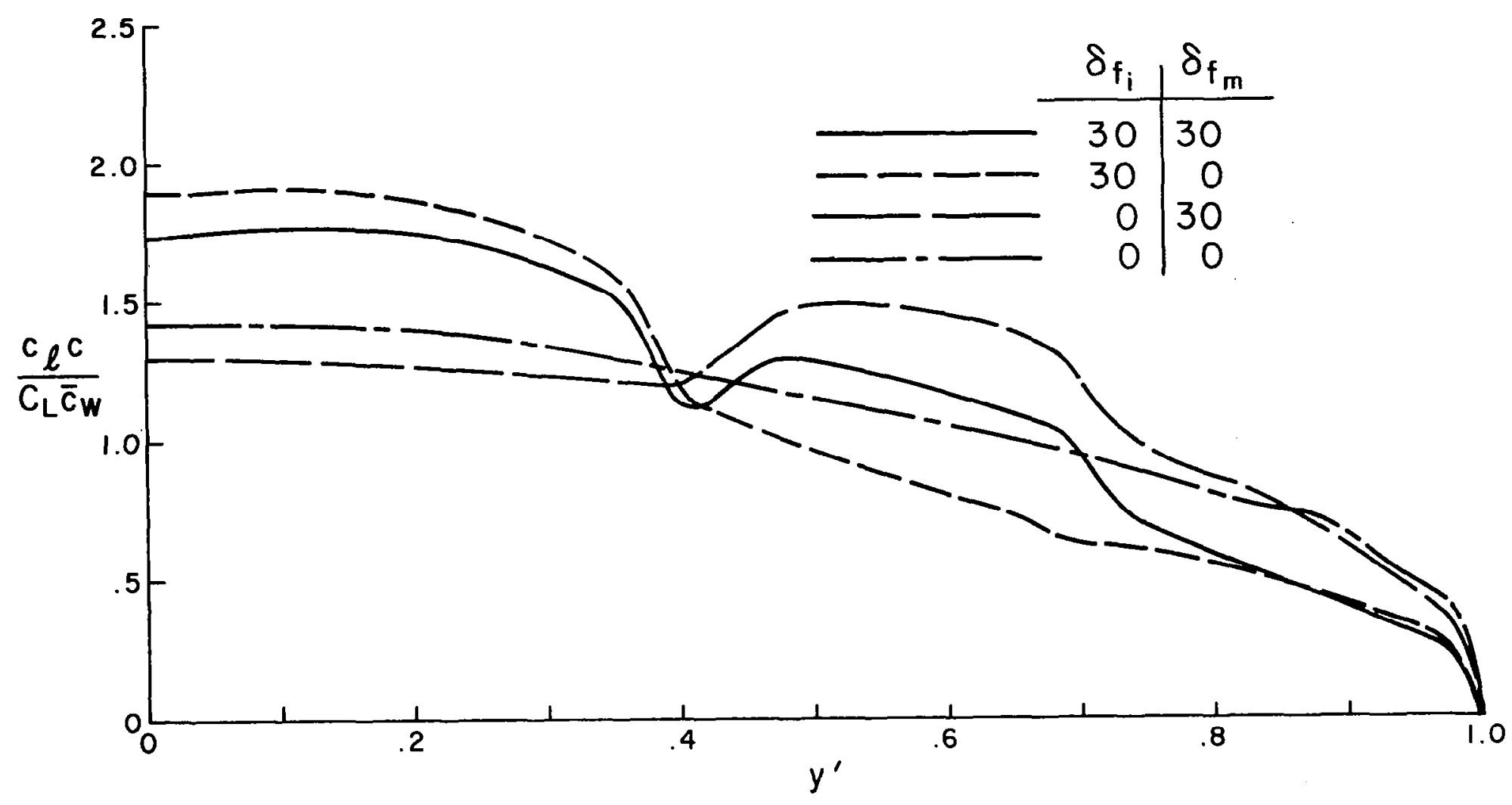

$\mathrm{G} \quad$ F1gure 5. $\begin{aligned} & \text { Computed B-747 wing lift distribution. } C_{L}=1.2 \text {, leading edge flaps } \\ & \text { extended }\end{aligned}$ 
Typical trajectories of the discrete vortices are presented and discussed in the next subsection, and we only present here the results obtained from our hazard criteria.

Shown below is the normalized maximum value of angular momentum in a circle whose diameter is that of the Learjet or DC-9 aircraft, at a downstream distance of $1.5 \mathrm{~km}$. The normalizing angular momentum was the value computed with $\delta_{f i}=\delta_{f m}=30^{\circ}$ (landing configuration) for the Learjet follower.

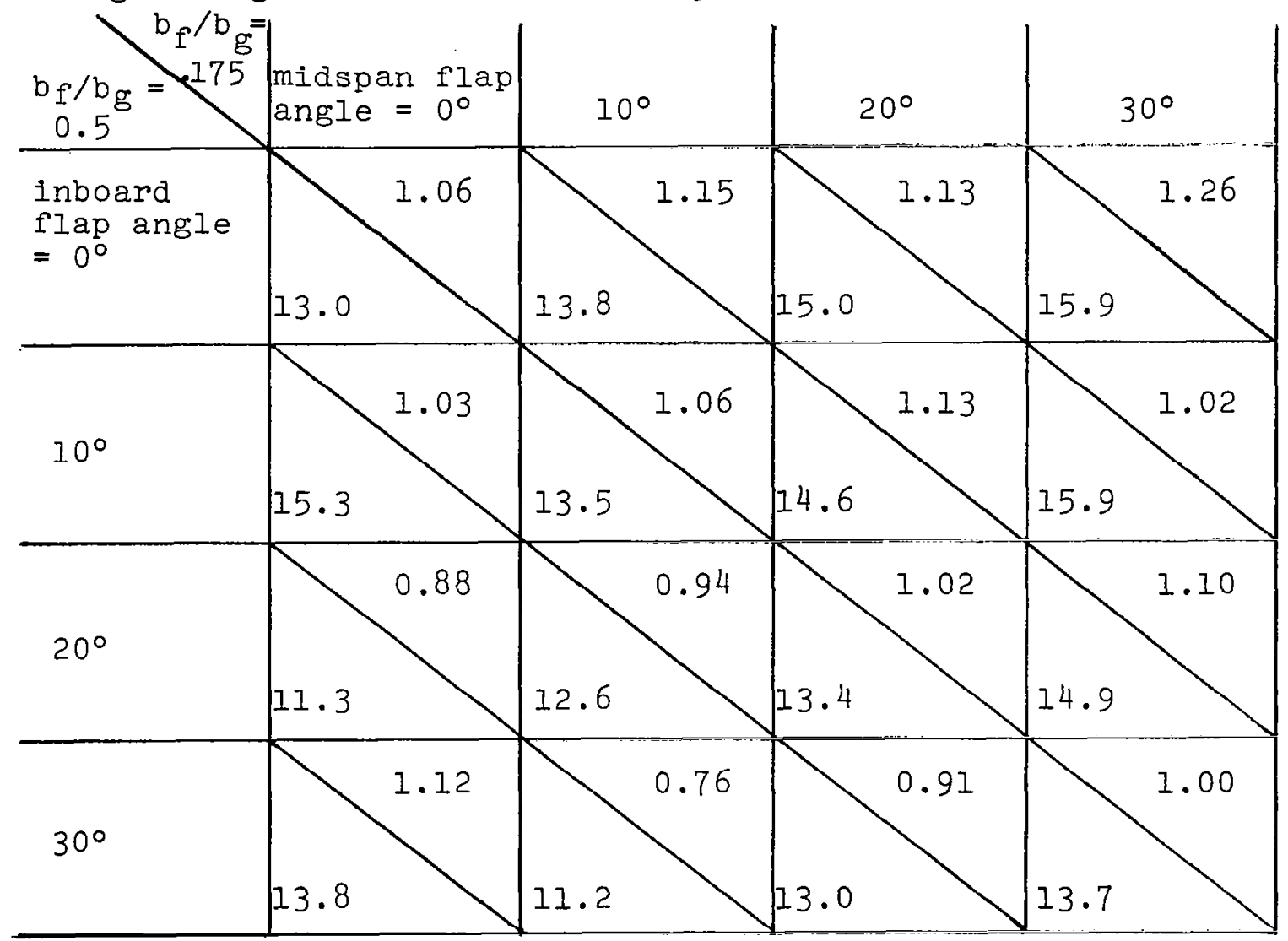

As a first general observation, the extreme variation of angular momentum over all configurations amounts to about $26 \%$. This is disconcerting in that towing tank tests (ref. 5) have shown at these downstream distances rolling moment reductions in excess of 50\%. This apparent discrepancy can be explained once we recall that point vortex computations, when carried out carefully, can at best only predict the convective redistribution of vorticity since, by assumption, diffusion is neglected. This is not to say that by 
using a suitably large number of point vortices the convective redistribution of mean velocity, as well as some turbulent structure, could not be duplicated for some time. What is missing in the cascade energy transfer process is dissipation at the smallest eddy scales. The question which is difficult to answer is, "For just how long (time) can a point vortex computation be expected to yield a realistic simulation?" For surely, as time becomes large, the kinetic energy in the real wake cross-plane goes to zero, while a point vortex computation conserves kinetic energy of the fluid indefinitely.

If we return to the question of numbers of point vortices needed, argument can be made that vortex placing should be of the order of the dissipation scale $\lambda$ which is a small dimension when compared to a relevant length scale in a vortex wake; say, the vortex spacing $d$. As a practical constraint, Rossow's (ref. 9) results using 40 vortices per vortex in merging computations seem to compare favorably with our extension of Moore and Saffman's analysis (ref. 8) shown in figure 3. However, we note that Rossow has reported to us in a private communication that the reduction in wake strength after merging is not very significant. This differs from the results of the full transport merging computations discussed in Section 5 .

Returning to the normalized angular momentum matrix does show the general trend that proceeding from the standard landing configuration, lessening the deflection of the midspan flap does, in general, reduce the angular momentum. The results for the $30^{\circ} / 0^{\circ}$ configuration do not yield the lowest hazard wake for the Learjet as has been observed in subscale and flight tests. However, the configurations bracketing the $30^{\circ} / 0^{\circ}$ configuration are the least hazardous. We speculate that the results for a $40^{\circ} / 0^{\circ}$ configuration would be even higher than the $30^{\circ} / 0^{\circ}$ result as more and more lift is carried on the inboard flap. Our calculations have shown that for the $30^{\circ} / 0^{\circ}$ configuration the flap vortex is about $30 \%$ larger than the tip vortex. The $20^{\circ} / 0^{\circ}$ configuration has the flap vortex strength approximately equal to the tip vortex strength--a design criterion which is shown in section 5 to be highly desirable in terms of maximizing vortex-vortex straining interactions.

The absolute minimum hazard configuration for the Learjet as identified from this computation is the $30^{\circ} / 10^{\circ}$, which is not easily explained at this time. We note only that in this case our computations have shown that the initial distribution of vortices 
initially broke into three clusters, two of which then merged, leaving two distinct vortex pairs in the wake. The angular momentum contributions of the clusters were about equal in the $1.5 \mathrm{~km}$. downstream position.

Curiously, the most hazardous wake for the Learjet is the $0^{\circ} / 30^{\circ}$ configuration, a situation which has been observed by NASA.

The results for the $\mathrm{DC}-9\left(\mathrm{~b}_{\mathrm{f}} / \mathrm{b}_{\mathrm{g}}=0.5\right)$ encountering aircraft are, in general, not surprising. The angular momentum is, of course, greater since the DC-9 wingspan is greater than the Learjet wing span. Configurations which give hazard alleviation to the Learjet also, in general, are less hazardous to the DC-9.

\subsection{Selected Studies Using Point Vortices}

We have tried to stress in the previous section that point vortex computations do indeed contribute to our understanding of the complicated interactions occurring in vortex wakes. We have also, however, expressed caution in interpreting results, particularly in terms of assessing the deintensification of aircraft wakes as a consequence of merging. When done carefully, point vortex computations can at best only model the convective redistribution of trailed vorticity. By assumption, diffusion of vorticity and dissipation of kinetic energy are neglected. Nevertheless, point vortex computations are valid over time scales in which diffusion can be neglected. In terms of aircraft wakes, a practical time limit is up to the time of vortex merging.

In this section, point vortex computations are used to obtain results which are relevant to vortex merging and wake interactions with the ground.

If given that a vortex wake in the immediate vicinity of a generating aircraft is composed of a tip and flap vortex, the likelihood of merging should increase if the separation between flap and tip vortices does not increase with distance downstream. Figures 6 and 7 . illustrate the trajectories of two differently structured wakes. The tight orbiting of flap and tip vortices in figure 6 will result in merging, while the weak interaction between flap and tip vortices shown in figure 7 may not result in merging.

For two-vortex-pair wakes, a qualitative prediction of whether merging may in fact occur can be obtained from a wake classification chart shown in figure 8 . When vortices are of like sign and fall in the region marked "remain together," one might expect that merging will eventually occur. On the other hand, wakes which fall in the region denoted "separate" may possibly remain unmerged. Wakes from real aircraft in a landing or takeoff 


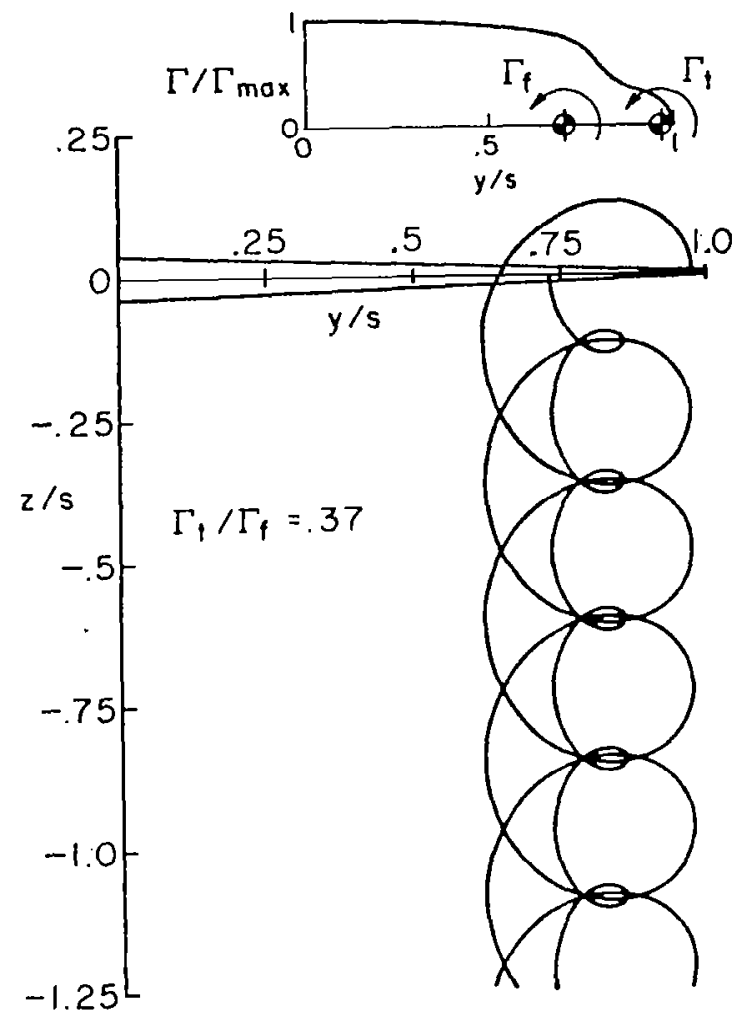

Figure 6. Vortex centroid locations as seen from downstream with strong interaction between neighboring vortices (ref. 2). 


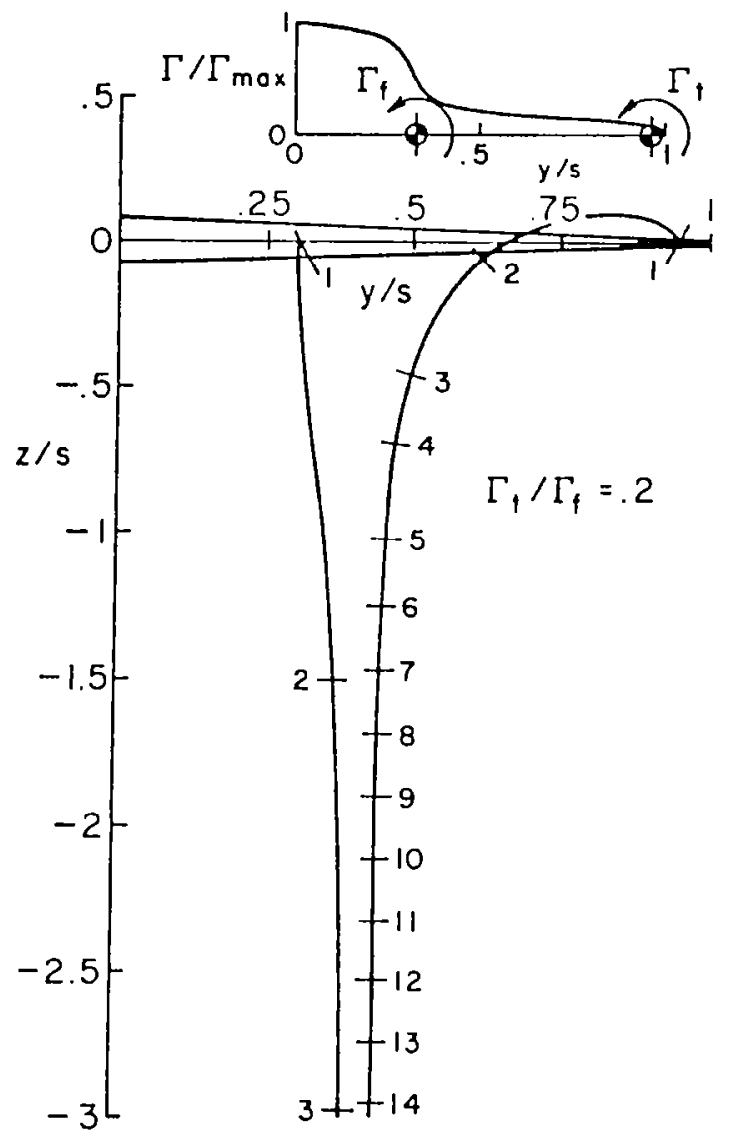

Figure 7. Vortex centroid locations as seen from downstream with weak interaction between likesigned vortices - pairs diverge. The time interval between consecutive integers is a constant (ref. 2). 


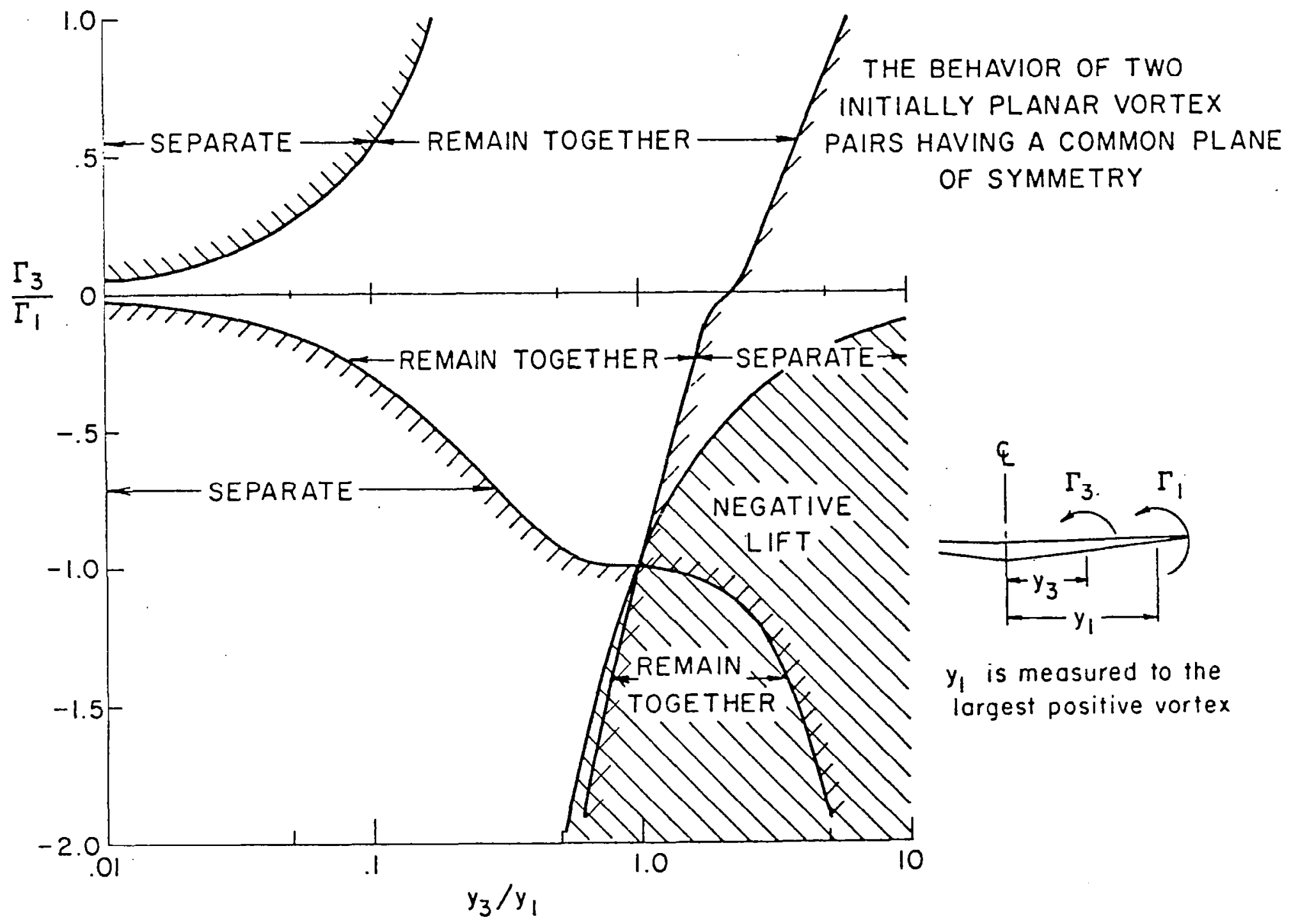

Figure 8. Wake classification chart for two-vortex-pair wakes (ref. 2) 
configuration, particularly with aircraft having a split flap arrangement, have far more structure than can be represented simply by two vortex pairs. In fact, the B-747 aircraft in a landing configuration trails six discrete pairs if the tail vortex is included (ref. 2).

At the next level of wake complexity, we consider a third vortex pair which can be used to model a fuselage vortex. We choose as a representative case the wake from the Landing/0 configuration studied by NASA. The load distribution shown in figure 9 has been taken from the work of Ciffone and Lonzo (ref. 12). Their code does attempt to predict loss of lift at the wing-fuselage junction. The Betz rollup recipe will predict rollup into three discrete vortices (ref. 2). The trajectories as viewed in a crossplane are shown in figure 10. As can be seen, the distance between the flap and tip vortices continually increases downstream. Ciffone and Lonzo conducted flow visualization experiments in a towing tank on this configuration and reported that merging did not occur. It is instructive to compute the separation between tip and flap vortices as a function of downstream distance while varying the strength of the fuselage vortex (fig. 11). By weakening the fuselage vortex, it is possible to reduce the separation between flap and tip vortices. The minimum separation between flap and tip vortices occurs when $\Gamma_{j} \simeq-0.3 \Gamma_{f}$. Further reduction in fuselage vortex strength serves only to again increase the distance between flap and tip vortices.

In figure 12, the sensitivity of distance between flap and tip vortices is investigated as a function of position of the fuselage vortex. By moving the fuselage vortex from $\bar{y}_{j} / s=0.125$ to 0.2 (a shift of $7.5 \%$ of the semispan), the flap and tip vortices can be brought to a distance of only $9 \%$ of the wing semispan.

The above computations serve to demonstrate the sensitivity of wake geometry to modest changes in wing load distribution. It is an unfortunate fact that techniques to give accurate predictions of load distributions are somewhat lacking, particularly for wings with flaps and slats deployed. The magnitude of the loss of lift at the wing-fuselage junction quite strongly influences wake geometry. There is now a need to know the wing load distribution more accurately than ever before.

The problem of multiple vortex interaction in the vicinity of the ground plane is examined next. It is during landing, inbound from the middle marker to touchdown, that is the most critical time in which to avoid a hazardous vortex encounter. Any vortex alleviation scheme will, therefore, have to operate in ground effect. In figure 13 are shown the computed trajectories of vortices in the wake of an aircraft having split flaps with only the inboard flap deployed. Vortices trailed from each half of the wing interact strongly and remain in very close proximity. In fact, the tip and 


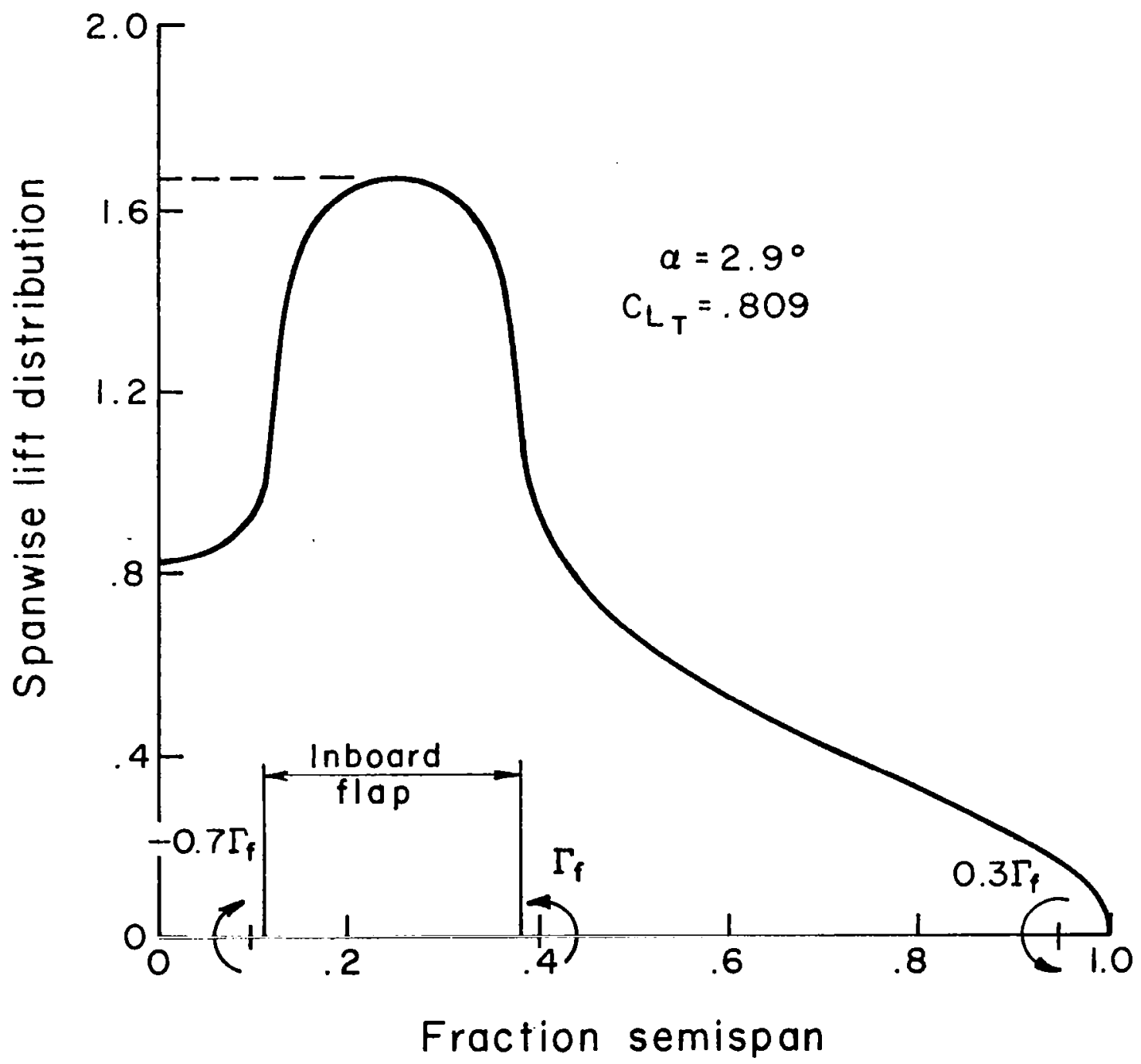

Figure 9. Predicted spanwise lift distribution; $L_{D(} / 0$ configuration (ref. 12). 


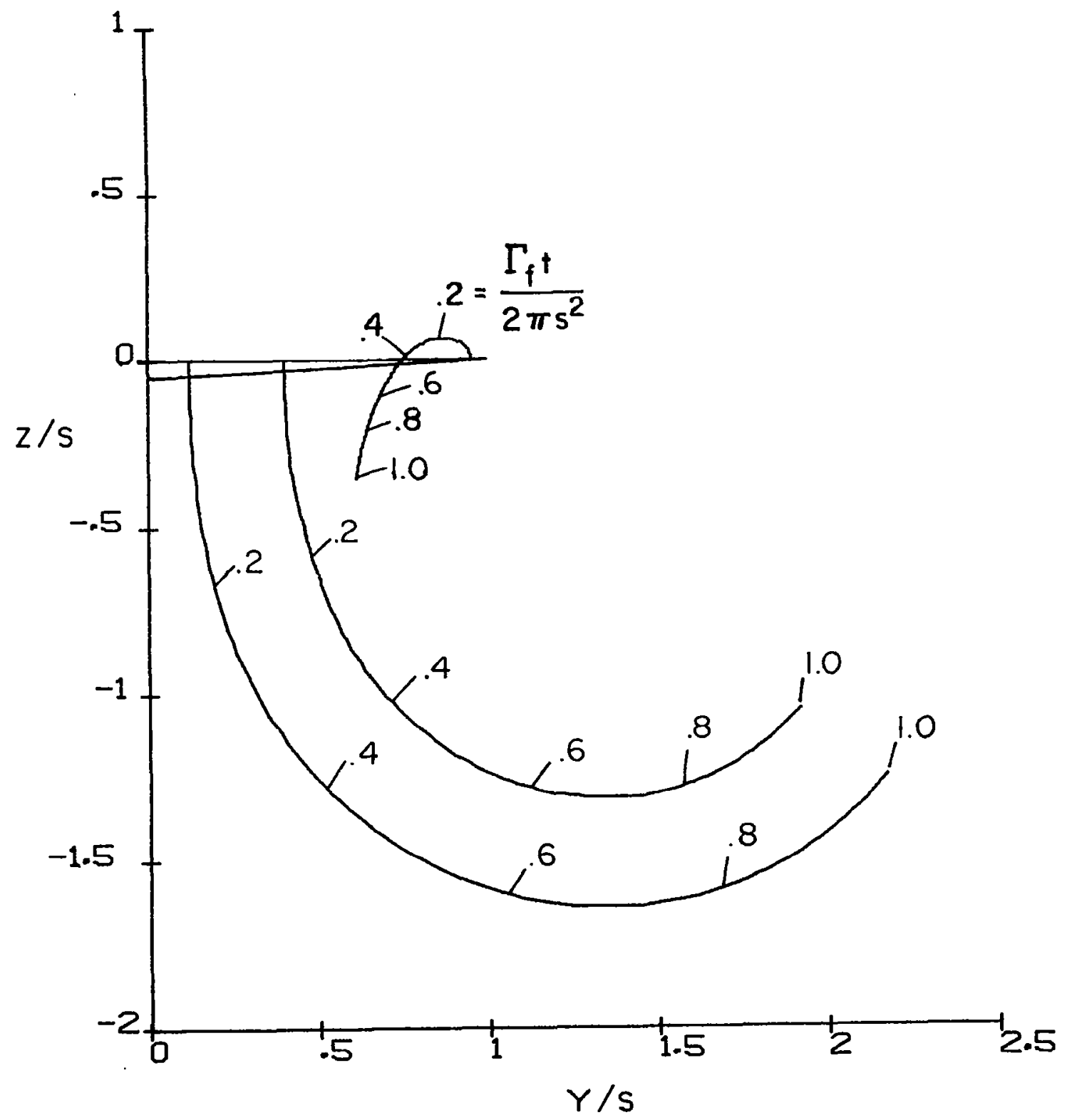

Flgure 10. Trajectories of the centroids of discrete vortices generated by the LDG/O configuration (see fig. 9). 


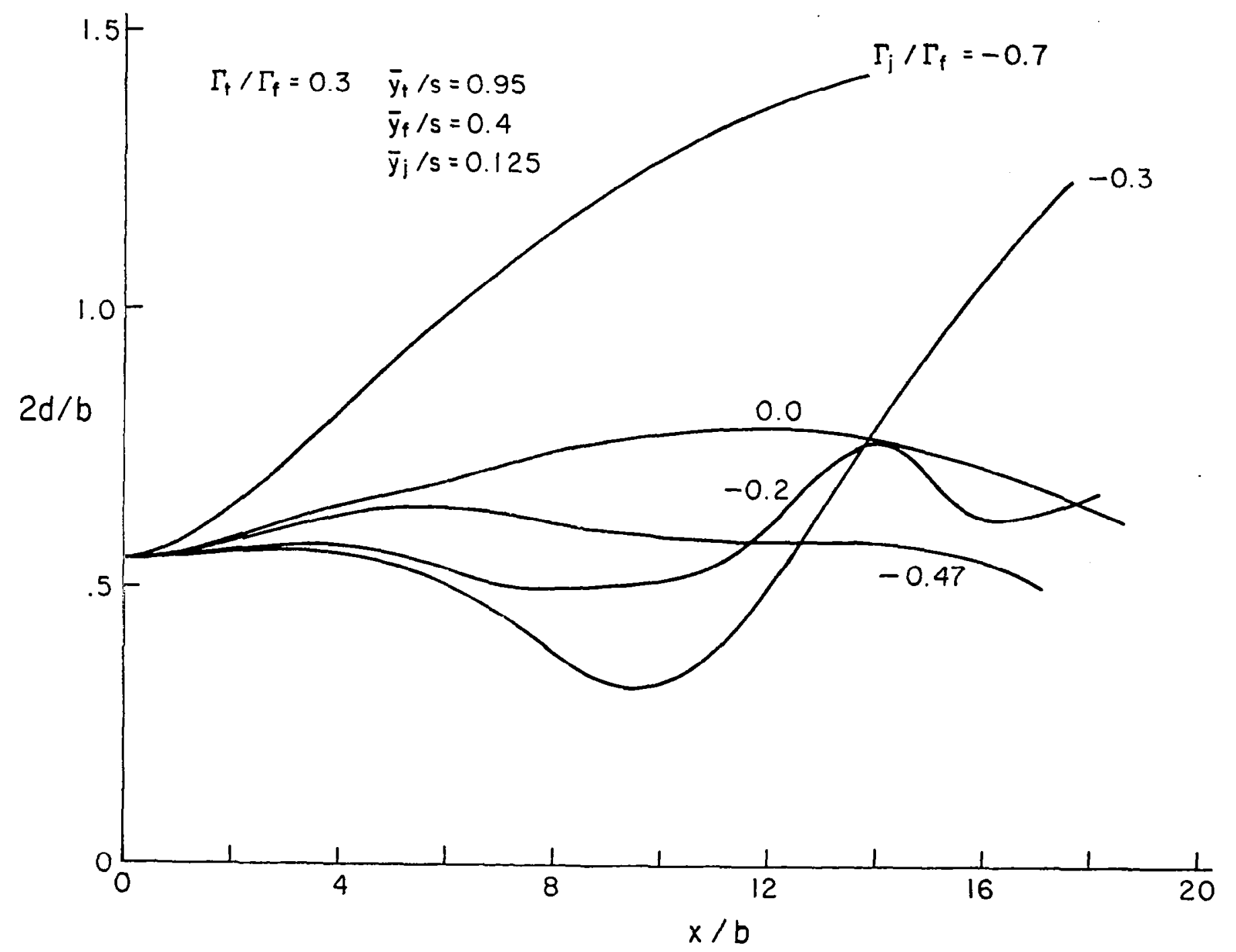

Figure 11. Sensitivity of the separation between tip and flap vortices as a function of the strength of the fuselage vortex $\Gamma_{1}$. The results from the LDG/0 configuration shown in figure 9 are labeled $\Gamma_{j}=-0.7 \Gamma_{f}$. 


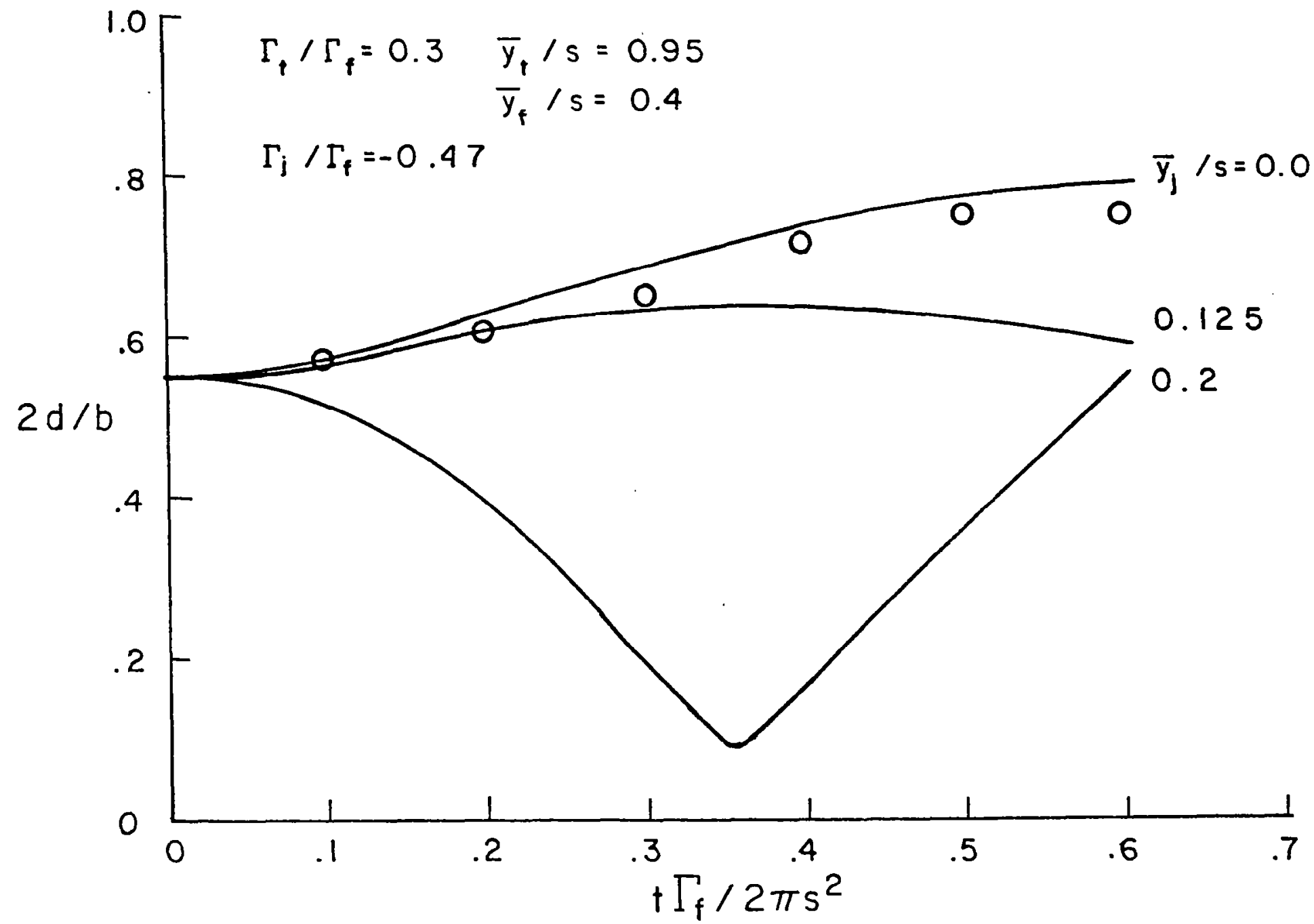

Figure 12. Sensitivity of the separation between tip and flap vortices as a function of the initial position of the fuselage vortex $\bar{y}_{j}$. 


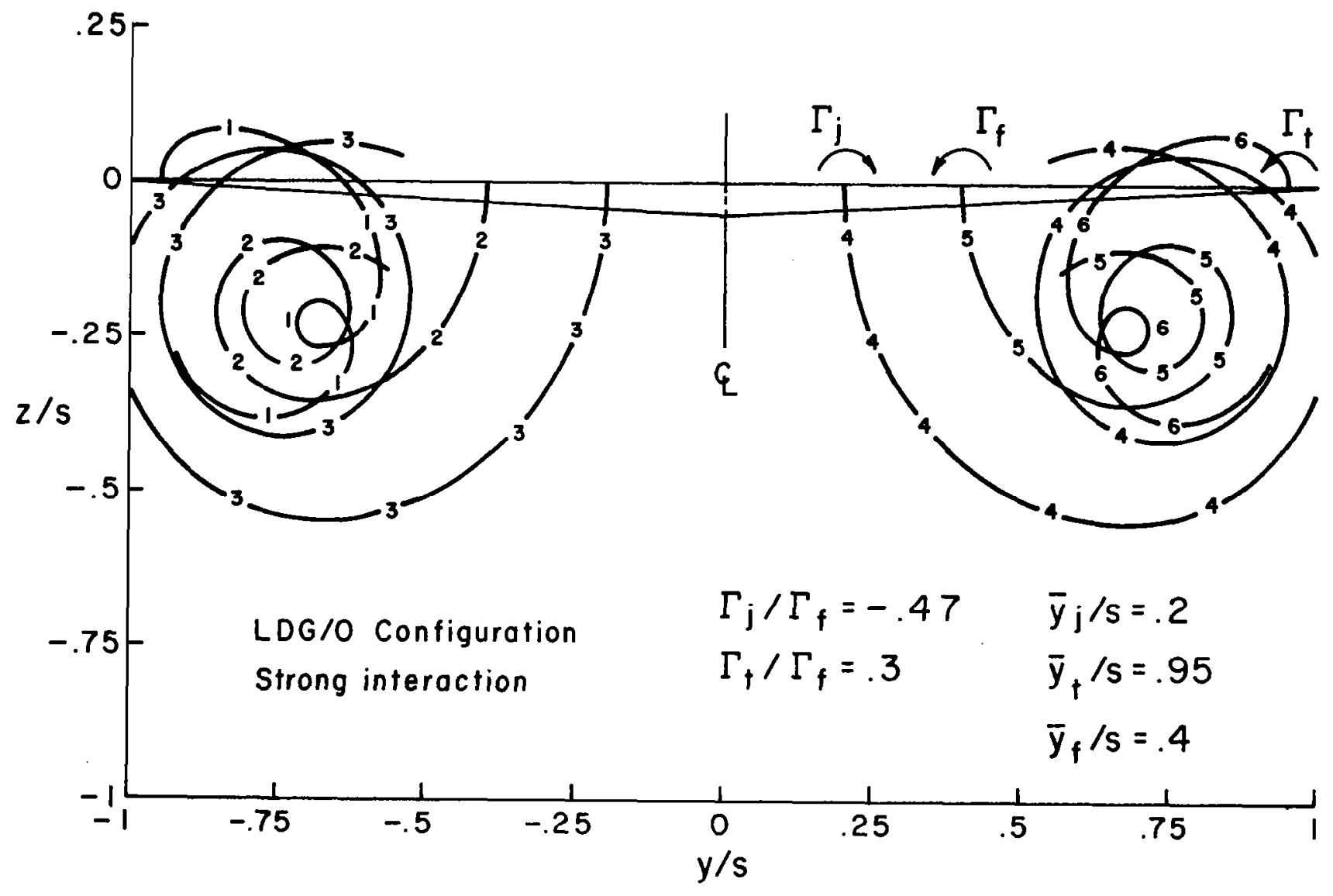


flap vortices which are initially separated by $0.55 \mathrm{~s}$ move to within about $0.08 \mathrm{~s}$ downstream. If the fuselage vortex is simply removed from the calculation, as shown in figure 14, the tightly wound vortex pattern is altered so that the distance between tip and flap vortices increases downstream. This again warns that the fuselage vortex can play an important role in the merging process. Now if the aircraft is moved to an elevation of $0.5 \mathrm{~s}$ above the ground and the fuselage vortex replaced, the vortex trajectories shown in figure 15 result. The results of these computations are summarized in figure 16. The above computations should warn that alleviation schemes involving vortex merging should be tested in ground effect to make certain that ground-plane interactions are not adverse to the merging phenomenon.

Before closing this section, we carry out several computations which suggest when it is appropriate to replace a distribution of point vortices modeling a discrete distribution of vorticity with one point vortex.

Figure 17 shows the modified Landing/0 configuration discussed in ref. 12 at a $C_{L} \simeq 0.8$. This load distribution was represented by forty discrete vortices of equal strength. The subsequent positions are shown in figure 18. Plotted also are the positions of the flap, tip, and fuselage vortices as obtained from a three-vortex-pair representation. These clusters are in reasonable agreement with the three-vortex-pair computations.

For a second computation, we remove the fuselage vortex from the modified Landing/0 configuration as shown by the dashed line on figure 17, still using forty equal-strength vortices to represent the trailed vorticity. In figure 19, a comparison of the manyvortex representation is made with a two-vortex-pair model. The results are quite acceptable.

Figure 20 shows the distance between the flap and tip vortex centroids, as computed above, with quite good agreement. The study shown in figure 12 indicates that moving the fuselage vortex to $20 \%$ of the semispan of the wing causes the flap and tip vortices to move into close proximity. The load distribution for the LDG/0 conflguration ( $\mathrm{fig}$. II) was altered inboard to create a fuselage vortex of strength $\Gamma_{j}=-0.47 \Gamma_{\mathrm{f}}$. As it turned out, the fuselage vortex centroid was located at $\bar{y} / s=0.185$. The separation between tip and flap vortices as a function of distance downstream is shown in figure 21 . For this configuration, minimum separation of $d / s \simeq 0.175$ is reasonably well predicted. However, the distances downstream at which this minimum is achieved do not compare favorably. When the fuselage vortex is removed so that flap and tip vortices move apart, the computation using a distribution of vortices again compares favorably with computations using one point vortex to represent each discrete vortex in the wake. The discrepancy when distributions move together lies in the incorrect approximation that the centroid of a given distribution of 


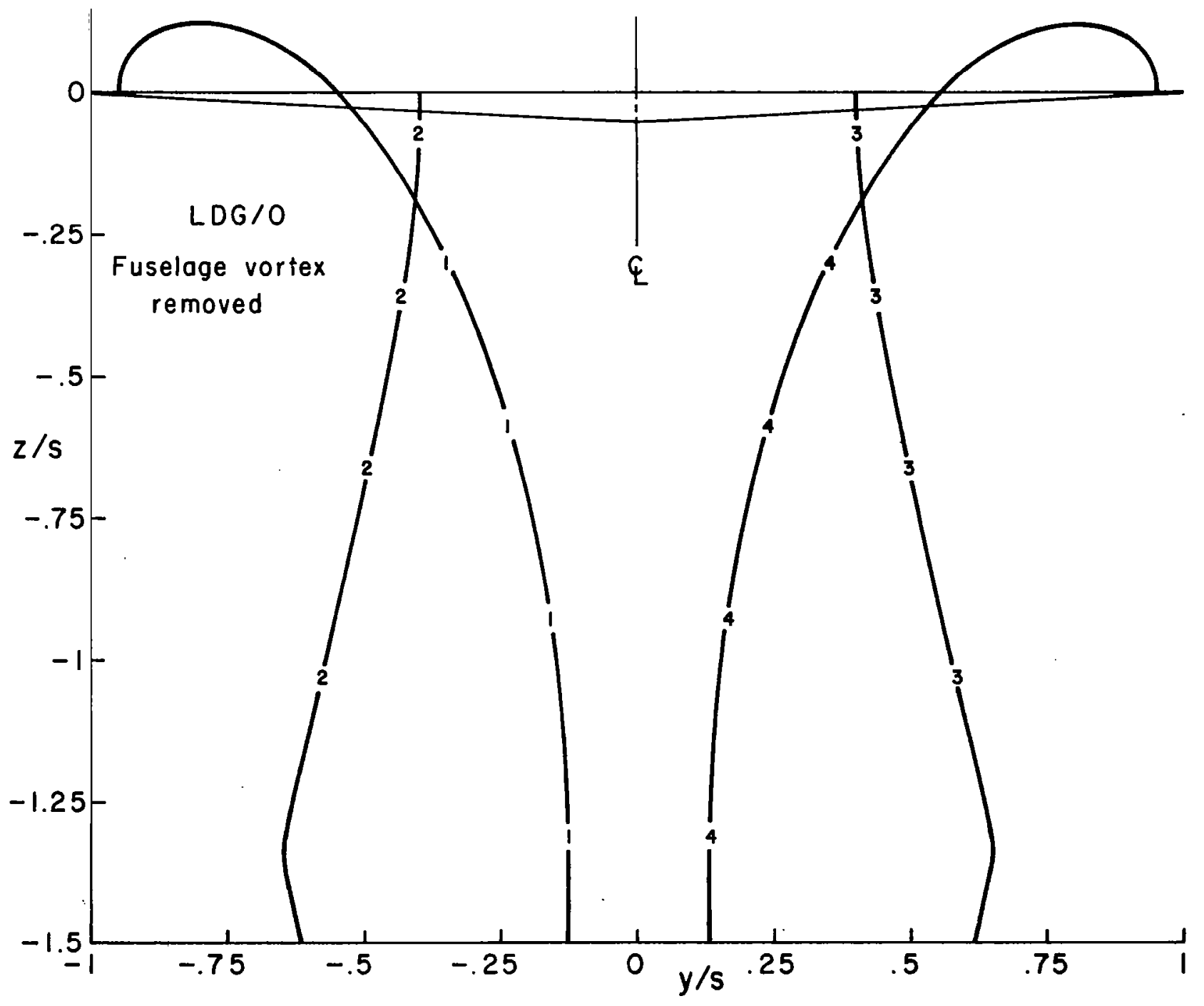

Figure 14. Vortex trajectories of the wake in figure 13 with the fuselage vortex removed 


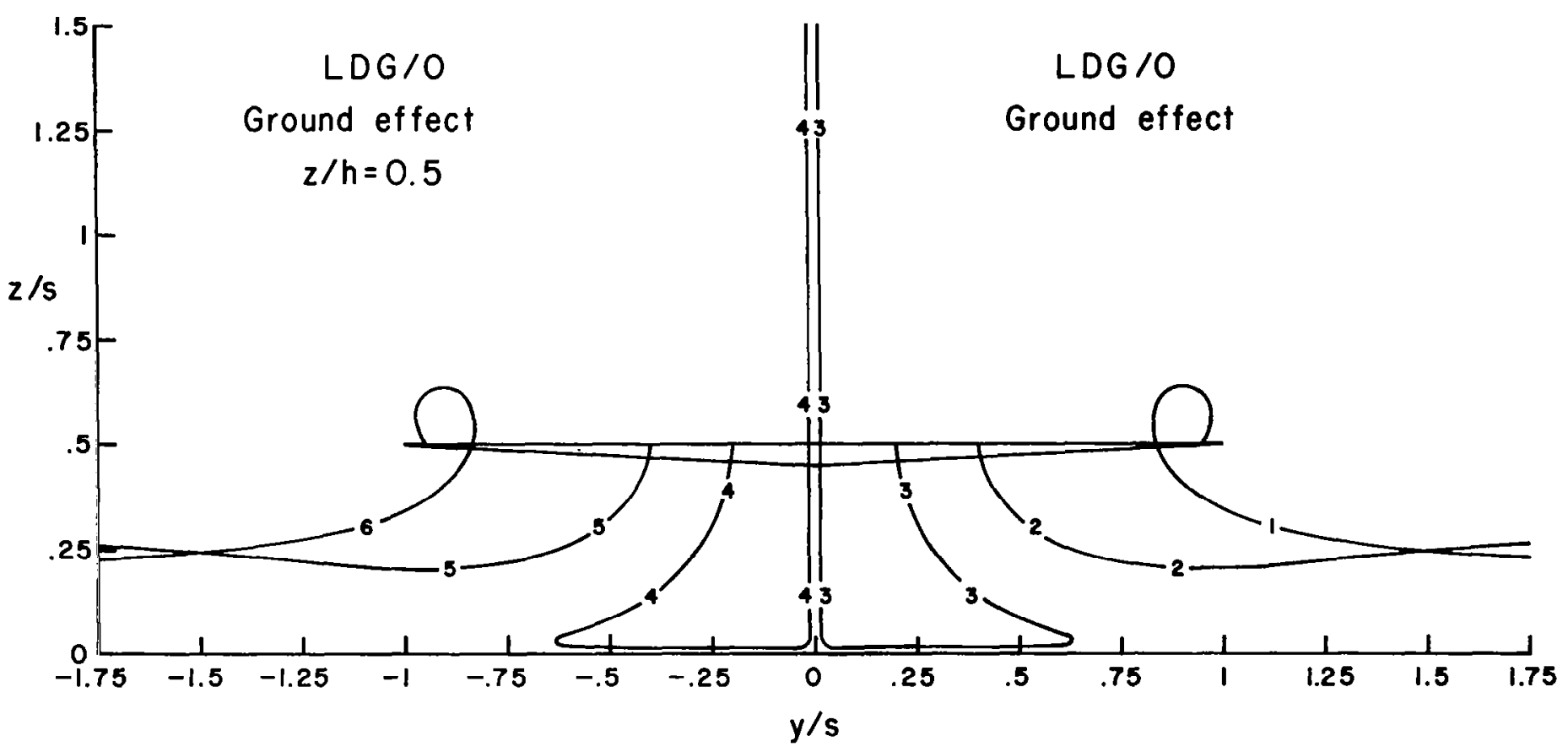

Figure 15. Vortex trajectorles of the wake in figure 13 in ground effect 


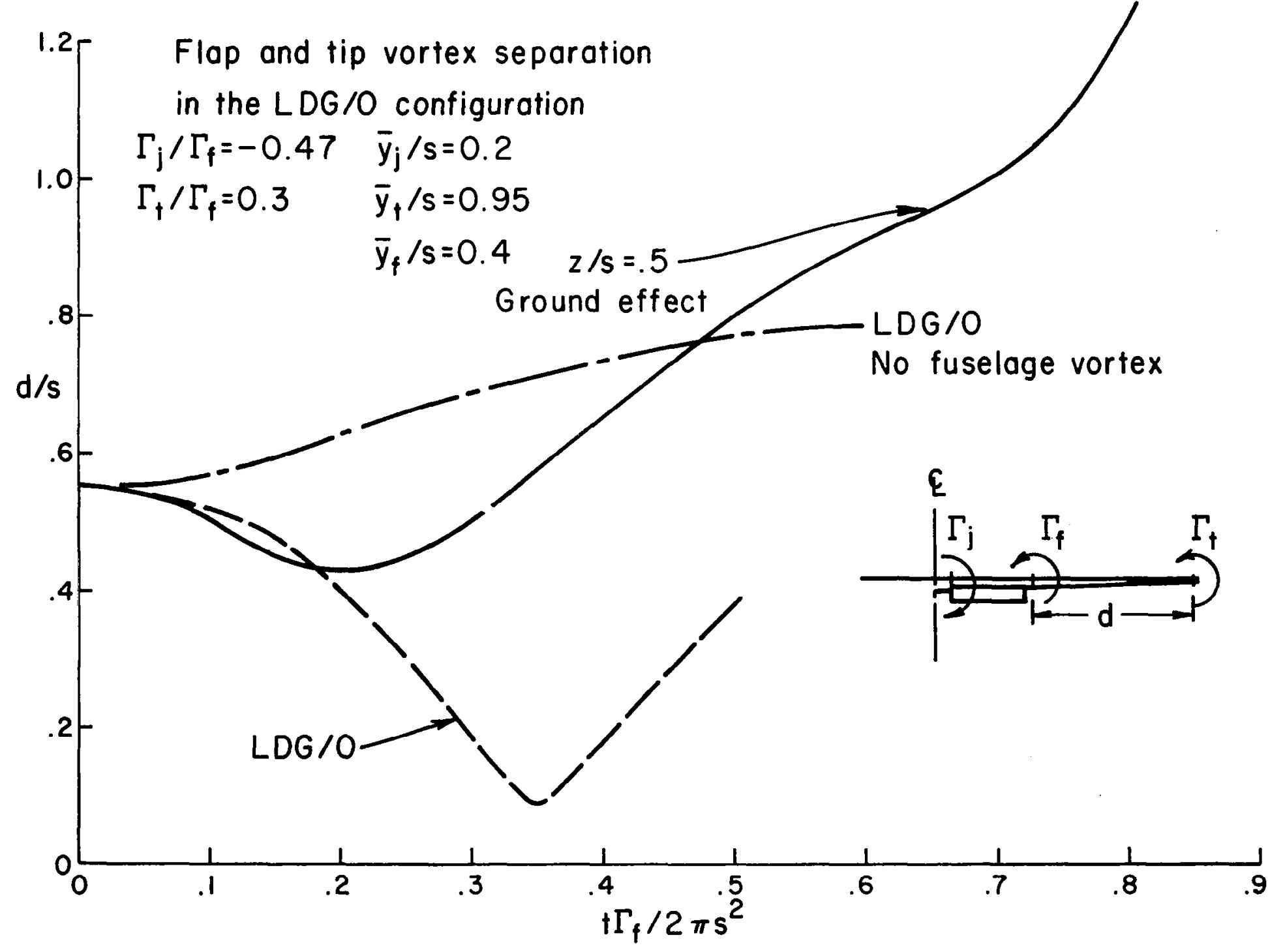

F1gure 16. Distance between the flap and tip vortices as a function of downstream distance 


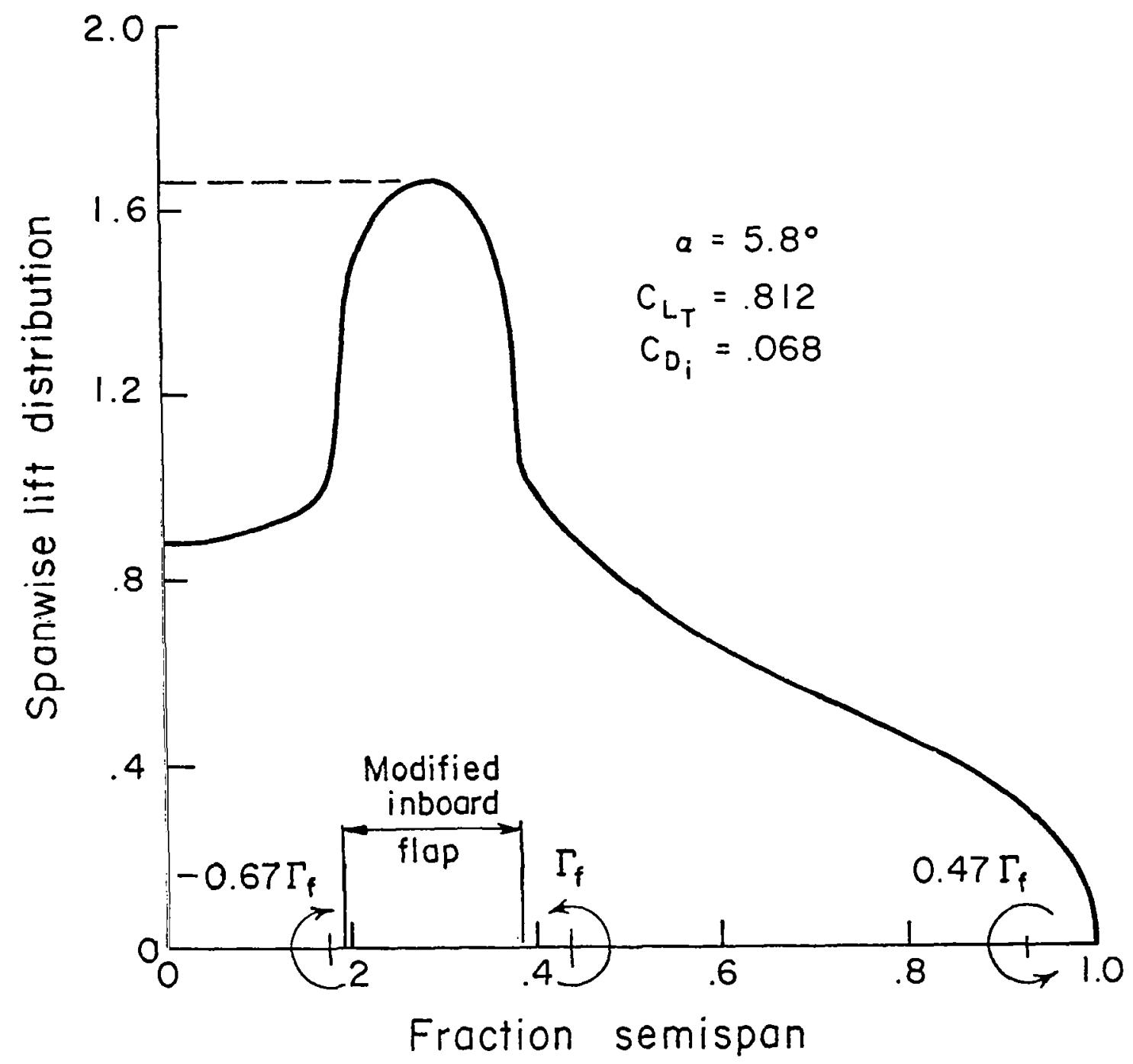
Figure 17. Predicted spanwise lift distribution, modified LDG/O configurations 


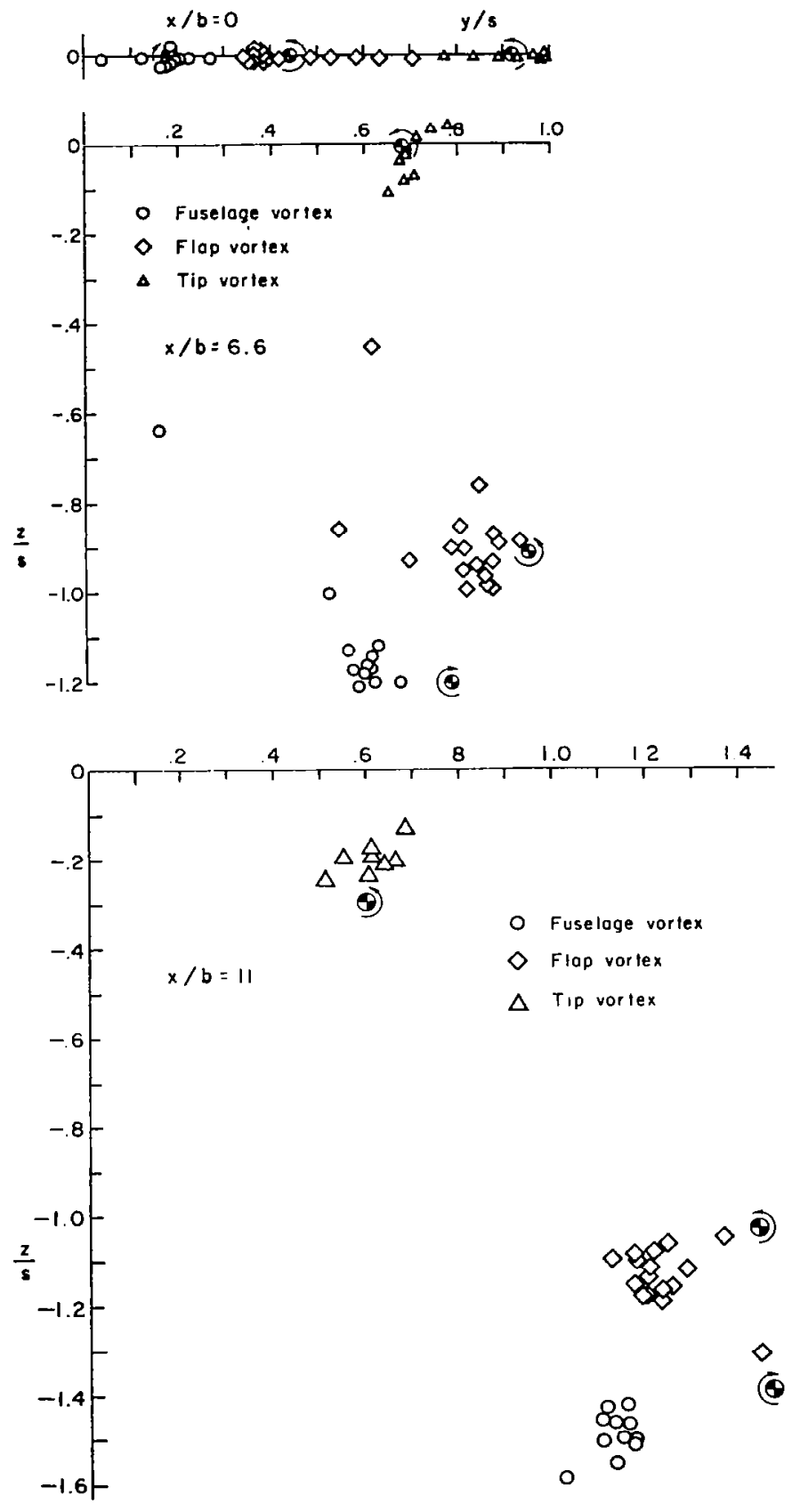

Figure 18. Comparison between using forty point vortices to represent the tralled sheet of vorticity and using three point vortices located at the centrold of the tip, flap, and fuselage vortices as shown in figure 17. $\rightarrow$ denotes centroid computed from the three-vortex computation. 


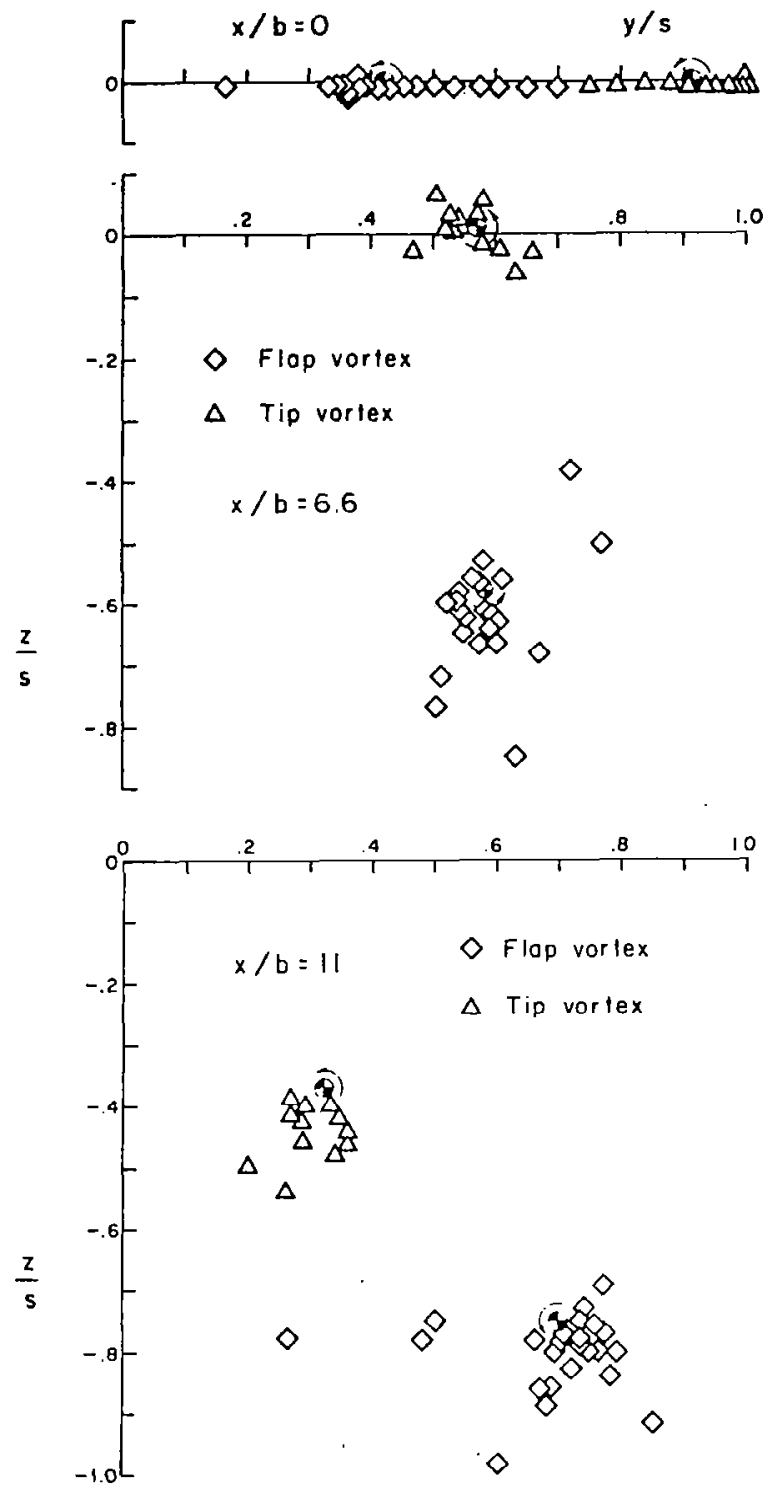

Figure 19. Comparison between using forty point vortices to represent the trailed sheet of vorticity and using two point vortices located at the centroid of the tip ancl flap vortices as showr in figure 17 (the fuselare vortex has been removerd). - denotes centroid computed from the two-vortex computation. 


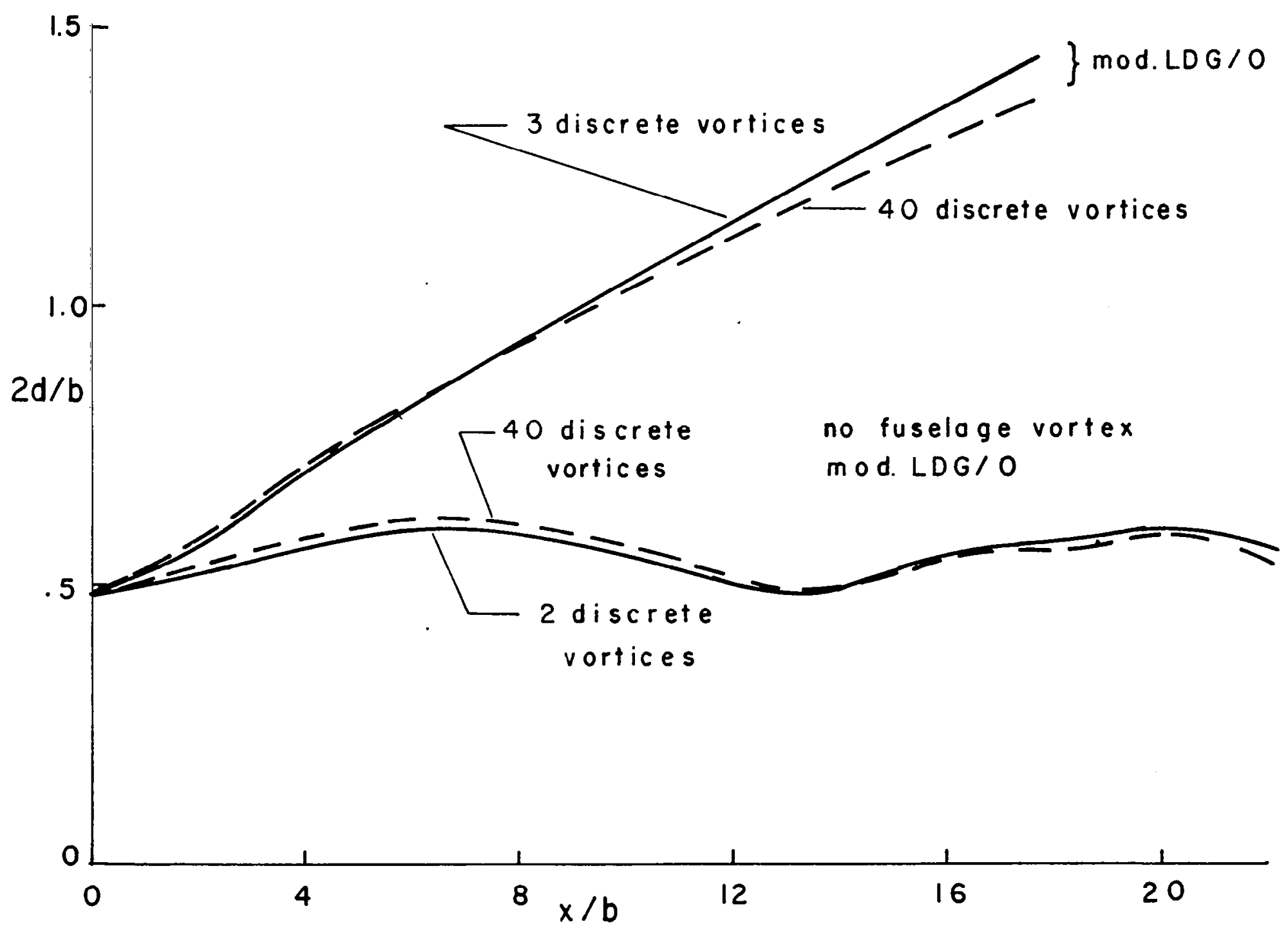

Figure 20. Comparison of the spacing between flap and tip vortices for the mod LDG/O configuration whose load distribution is shown in figure 17. 


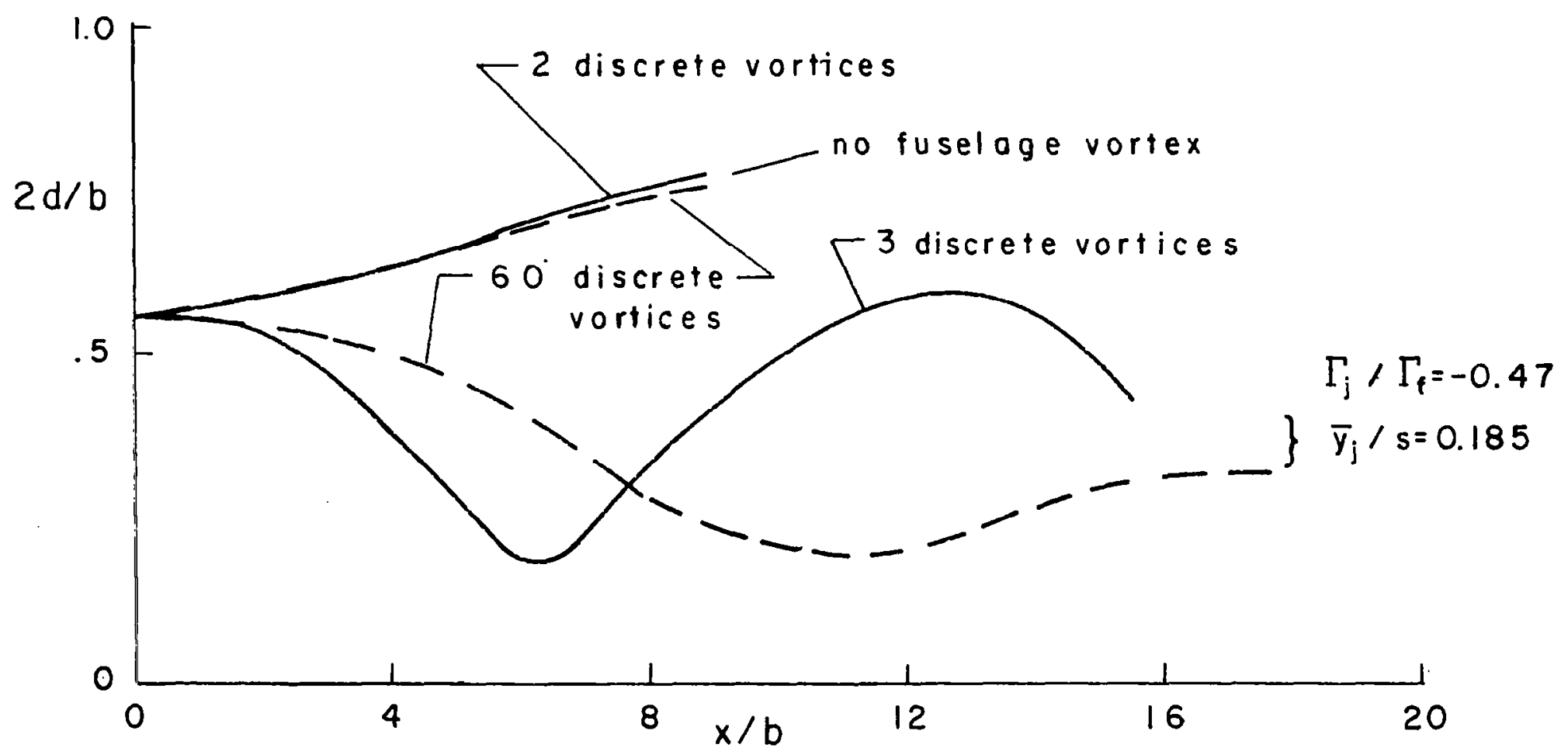

Figure 21. Comparison of the spacing between flap and tip vortices for an inboard altered LDG/O lift distribution. 
vorticity moves at the velocity induced at its centroid by other concentrations. In reference 2 we show that the velocity of the centroid of a bounded region of vorticity in an infinite fluid is given by

$$
\frac{d \vec{t}}{d t}=\frac{\int \vec{U}_{0} \zeta d A}{\Gamma}
$$

where $\vec{U}_{0}$ is the velocity field induced by the presence of all other concentrations of vorticity in the fluid other than the one whose motion is being computed. As two distributions come close together, $U_{0}$ may vary significantly over $\zeta$, and the motion of the centroid is not simply the fluid velocity at the centroid induced by all other concentrations of vorticity.

It has been our purpose in this section to point out that simple discrete vortex computations can be used to assess quickly whether particular lift distributions will result in a multiple vortex wake which might merge. When vortices of like sign remain in proximity to each other, the merging process must eventually occur. An estimation of the deintensification as a result of merging requires the use of a transportive fluid model, such as that presented in section 3. In sum, inviscid computations indicate that small changes in aircraft configuration and, hence, wing lift distribution can have a profound effect on wake structure. More accurate knowledge of lift distributions on commercial jetIners, in the landing and takeoff configurations, with and without landing gear deployed, would aid greatly in understanding the dynamics of aircraft vortex wakes. 
3. THE TRANSPORT MODEL

A second-order closure of the Reynolds stress equations has been under development at A.R.A.P. for many years. The current modeled system of equations is one which has been used to predict successfully both quantitative and qualitative features of complex turbulent flow fields (refs. 14-17). In this section, we review the invariant technique of turbulent modeling. The reader can find a more comprehensive development in references 16 and 17 . Vortex wake calculations solving the equations developed here will be presented in section 5 of this report.

The Navier-Stokes equations govern the solution to well-posed turbulent flow problems which are of interest in vortex wakes. Unfortunately, full numerical unsteady solutions of these equations are beyond the current technical computer capacities. Therefore, we try to obtain solutions for the ensemble-averaged mean field and ensemble-averaged second-order correlations.

We begin by writing every flow variable with a mean and fluctuating part $\left(e \cdot g ., \underline{W}=U_{i}+u_{i}\right)$. We then make formal substitution into the equations of motion and ensemble average to obtain the mean flow equations.

Continuity

$$
\frac{\partial U_{i}}{\partial x_{i}}=0
$$

Momentum

$\frac{\partial U_{i}}{\partial t}+U_{j} \frac{\partial U_{i}}{\partial x_{j}}=-\frac{\partial \overline{u_{i} u_{j}}}{\partial x_{j}}+\frac{\partial}{\partial x_{j}}\left(\nu \frac{\partial U_{i}}{\partial x_{j}}\right)-\frac{I}{\rho} \frac{\partial P}{\partial x_{i}}+\frac{g_{i}}{\theta_{0}}\left(\theta-\theta_{0}\right)$

Energy

$$
\frac{\partial \theta}{\partial t}+u_{j} \frac{\partial \theta}{\partial x_{j}}=-\frac{\partial \overline{u_{i} \theta}}{\partial x_{i}}+\frac{\partial}{\partial x_{i}}\left(v \frac{\partial \theta}{\partial x_{i}}\right)
$$

We have assumed that the flow is incompressible but have permitted small changes in density due to changes in temperature. The only effect of density variation included is in the gravitational body force term in the momentum equation (ref. 16)( the Boussinesq approximation (ref. 18)). In vortex wakes, this body force term adds buoyancy for hot engine exhausts. The problem with eqs. (8)-(10) is that our decomposition into mean and 
fluctuating parts has led to the addition of unknown correlations $\bar{u}_{i} u_{j}$ and $\overline{u_{i} \theta}$ which makes the system of equations incomplete. The essence of first-order turbulent closure is to model these new terms as functions of first derivatives, e.g.,

$$
\overline{u_{i} u_{j}}=-K \frac{\partial U_{i}}{\partial x_{j}}
$$

where $K$ is an appropriate "eddy viscosity coefficient." This technique closes the system of equations but, in a great many cases, places a large, uncompromising burden upon the selection, use, and form of K--certainly rarely a constant.

Recognizing this problem, we endeavor to find "exact" expressions for $\overline{u_{j} u_{j}}$ and $\overline{u_{j} \theta}$. This can be done by subtracting the mean equations ( 9 ) and (10) from the complete equations, leaving equations for $u_{i}$ and $\theta$ which can be premultiplied and averaged to yield the proper exact expressions (ref. 16).

$$
\begin{aligned}
& \frac{\partial \overline{u_{i} u_{j}}}{\partial t}+U_{k} \frac{\partial \overline{u_{i} u_{j}}}{\partial x_{k}}=-\overline{u_{i} u_{k}} \frac{\partial U_{j}}{\partial x_{k}}-\overline{u_{j} u_{k}} \frac{\partial U_{i}}{\partial x_{k}}+\frac{g_{i}}{\theta_{o}} \overline{u_{j} \theta}+\frac{g_{j}}{\theta_{o}} \overline{u_{i} \theta} \\
& -\frac{\partial}{\partial x_{k}}\left(\overline{u_{k} u_{i} u_{j}}\right)-\overline{\frac{u_{i}}{\rho} \frac{\partial P}{\partial x_{j}}}-\overline{\frac{u_{j}}{\rho} \frac{\partial P}{\partial x_{i}}}
\end{aligned}
$$

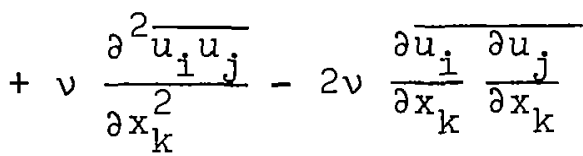

$$
\begin{aligned}
& \frac{\partial \overline{u_{i} \theta}}{\partial t}+U_{k} \frac{\partial \overline{u_{i} \theta}}{\partial x_{k}}=-\overline{u_{i} u_{k}} \frac{\partial \theta}{\partial x_{k}}-\overline{u_{k} \theta} \frac{\partial U_{i}}{\partial x_{k}}+\frac{g_{i}}{\theta_{0}} \overline{\theta^{2}} \\
& -\frac{\partial}{\partial x_{k}}\left(\overline{u_{i} u_{k} \theta}\right)-\overline{\frac{\theta}{\rho} \frac{\partial P}{\partial x_{1}}} \\
& +v \frac{\partial^{2} \overline{u_{i} \theta}}{\partial x_{k}^{2}}-2 v \frac{\partial \overline{u_{i}}}{\partial x_{k}} \frac{\partial \theta}{\partial x_{k}}
\end{aligned}
$$




$$
\frac{\partial \overline{\theta^{2}}}{\partial t}+u_{k} \frac{\partial \overline{\theta^{2}}}{\partial x_{k}}=-2 \overline{u_{k} \theta} \frac{\partial \theta}{\partial x_{k}}-\frac{\partial}{\partial x_{k}}\left(\overline{u_{k} \theta^{2}}\right)+v \frac{\partial^{2} \overline{\theta^{2}}}{\partial x_{k}^{2}}-2 v \frac{\partial \bar{\theta}}{\partial x_{k}} \frac{\partial \theta}{\partial x_{k}}
$$

Unfortunately, this set still does not permit closure because of the presence of third-order velocity correlations:

$$
\frac{\partial}{\partial x_{k}}\left(\overline{u_{k} u_{i} u_{j}}\right), \frac{\partial}{\partial x_{k}}\left(\overline{u_{i} u_{k} \theta}\right), \frac{\partial}{\partial x_{k}}\left(\overline{u_{k} \theta^{2}}\right)
$$

pressure correlations:

$$
\overline{\frac{u_{i}}{\rho} \frac{\partial P}{\partial x_{j}}}+\overline{\frac{u_{j}}{\rho} \frac{\partial P}{\partial x_{i}}}, \overline{\frac{\theta}{\rho} \frac{\partial P}{\partial x_{i}}}
$$

and dissipation terms:

$$
\frac{\partial \overline{u_{i}}}{\partial x_{k}} \frac{\partial u_{i}}{\partial x_{k}}, \frac{\partial \overline{u_{i}} \frac{\partial \theta}{\partial x_{k}}}{\partial x_{k}}, \frac{\partial \theta}{\partial x_{k}} \frac{\partial \theta}{\partial x_{k}}
$$

In fact, for every additional new equation written, we would introduce successively higher-order turbulent correlations. Secondorder closure stops here and attempts to model the above-noted expressions in some rational manner. That many other closure model schemes exist goes without saying (refs. 19, 20).

We strive for an "invariant" model. Our definition may be taken in two ways: one, any expression that replaces any of the above-noted expressions must exhibit the same tensor symmetry and dimensionality as the replaced term; two, our final simple model with its inevitable modeling constants must be consistently and fairly applied without modifications to a wide variety of flow geometries and initial conditions.

We choose to replace these higher-order correlations by appropriate second-order correlations and their gradients, mean flow gradients, and proper scaling and modeling constants. The velocity correlations, eq. (15) can be diffusive in nature and are therefore replaced by gradients of the appropriate second-order correlation. Thus, for the term involving $u_{k} u_{1} u_{j}$, we write 


$$
-\frac{\partial}{\partial x_{k}}\left(\overline{u_{k} u_{i} u_{j}}\right) \sim v_{c} \frac{\partial}{\partial x_{k}}\left(q \Lambda \frac{\partial \overline{u_{i} u_{j}}}{\partial x_{k}}\right)
$$

where we have introduced the length scale $\Lambda$ and velocity scale $\mathrm{q}$ to make the substitution dimensionally sound. The scale $\Lambda$ (the macroscale) measures the breadth of the turbulence under study; its description would generally vary from flow application to application. The velocity scale $q$ is taken as the root-meansquare of twice the turbulent kinetic energy

$$
q^{2}=\overline{u_{1} u_{i}}=\overline{u u}+\overline{v v}+\overline{w w}
$$

The constant $v_{c}$ adjusts any magnitude difference between the modeled and unmodeled terms and is the "diffusion coefficient" of the invariant model.

The pressure correlations, eq. (16) may be rewritten to give

$$
\overline{u_{i} \frac{\partial P}{\partial x_{j}}}+\overline{u_{j} \frac{\partial P}{\partial x_{i}}}=\frac{\partial \overline{u_{i} P}}{\partial x_{j}}+\frac{\partial \overline{u_{j}} \bar{P}}{\partial x_{i}}-\overline{P\left(\frac{\partial u_{i}}{\partial x_{j}}+\frac{\partial u_{j}}{\partial x_{i}}\right)}
$$

The first two terms on the right-hand side are diffusive and are absorbed into the velocity correlation model, eq. (18). The remaining term is a tendency-towards-isotropy term whereby the fluctuations attempt to redistribute turbulent energy between the three energy components. Following Rotta (ref. 2l)

$$
-\overline{\frac{p}{\rho}\left(\frac{\partial u_{i}}{\partial x_{j}}+\frac{\partial u_{j}}{\partial x_{i}}\right)}=\frac{q}{\Lambda}\left(\overline{u_{i} u_{j}}-\delta_{i j} \frac{q^{2}}{3}\right)
$$

The dissipation terms are modeled so as to be isotropic, so that

$$
v \overline{\frac{\partial u_{i}}{\partial x_{k}} \frac{\partial u_{1}}{\partial x_{k}}}=\delta_{i j} \frac{b q^{3}}{3 \Lambda}
$$

where $b$ is an additional model constant.

Upon substitution of models, eqs. (18)-(21), the complete set of Reynolds stress equations becomes 


$$
\begin{aligned}
& \frac{\partial \overline{u_{i} u_{j}}}{\partial t}+u_{k} \frac{\partial \overline{u_{i} u_{j}}}{\partial x_{k}}=-\overline{u_{i} u_{k}} \frac{\partial U_{j}}{\partial x_{k}}-\overline{u_{j} u_{k}} \frac{\partial U_{1}}{\partial x_{k}}+\frac{g_{1}}{\theta_{0}} \overline{u_{j} \theta}+\frac{g_{j}}{\theta_{0}} \overline{u_{i} \theta} \\
& +v_{c} \frac{\partial}{\partial x_{k}}\left(q \Lambda \frac{\partial \overline{u_{i} u_{j}}}{\partial x_{k}}\right)-\frac{q}{\Lambda}\left(\overline{u_{i} u_{j}}-\delta_{1 j} \frac{q^{2}}{3}\right) \\
& +v \frac{\partial^{2} \overline{u_{i} u_{j}}}{\partial x_{k}^{2}}-\delta_{i j} \frac{2 b q^{3}}{3 \Lambda} \\
& \frac{\partial \overline{u_{i} \theta}}{\partial t}+u_{k} \frac{\partial \overline{u_{i} \theta}}{\partial x_{k}}=-\overline{u_{i} u_{k}} \frac{\partial \theta}{\partial x_{k}}-\overline{u_{k} \theta} \frac{\partial U_{i}}{\partial x_{k}}+\frac{g_{i}}{\theta_{0}} \overline{\theta^{2}} \\
& +v_{c} \frac{\partial}{\partial x_{k}}\left(q \Lambda \frac{\partial \overline{u_{i} \theta}}{\partial x_{k}}\right)-\frac{A q}{\Lambda} \overline{u_{i} \theta}+v \frac{\partial^{2} \overline{u_{i} \theta}}{\partial x_{k}^{2}} \\
& \frac{\partial \theta^{2}}{\partial t}+u_{k} \frac{\partial \theta^{2}}{\partial x_{k}}=-2 \overline{u_{k} \theta} \frac{\partial \theta}{\partial x_{k}} \\
& +v_{c} \frac{\partial}{\partial x_{k}}\left(q \Lambda \frac{\partial \theta^{2}}{\partial x_{k}}\right)+v \frac{\partial^{2} \bar{\theta}^{2}}{\partial x_{k}^{2}}-\frac{2 b s q \bar{\theta}^{2}}{\Lambda}
\end{aligned}
$$

Further considerations have prompted us to add the two extra model constants A (in the tendency-towards-isotropy term of the velocitytemperature correlation equations) and $s$ (in the dissipation terms of the temperature-temperature correlations). The fundamental velocity scale $q$ may be determined by summing eq. (22) for $1=j=1,2,3$ to obtain a differential equation for $q^{2}$. The fundamental length scale $\Lambda$ may be specified by some algebraic relationship peculiar to the flow under consideration, but generality comes by writing a differential scale equation and actually solving for $\Lambda$ as we solve for $\overline{u_{i} u_{j}}, \overline{u_{i} \theta}$, and $\overline{\theta^{2}}$. The process is admittedly more difficult because the scale equation does not follow directly from the equations of motion. However, assembling the terms most normally felt to influence the scale results in the equation used at A.R.A.P. (ref. 17): 


$$
\begin{aligned}
\frac{\partial \Lambda}{\partial t}+U_{k} \frac{\partial \Lambda}{\partial x_{k}} & =-s_{I} \frac{\Lambda}{q^{2}} \overline{u_{i} u_{k}} \frac{\partial U_{i}}{\partial x_{k}}-s_{2} q \\
& +v_{c} \frac{\partial}{\partial x_{k}}\left(q \Lambda \frac{\partial \Lambda}{\partial x_{k}}\right)-\frac{s_{3}}{q}\left(\frac{\partial q \Lambda}{\partial x_{k}}\right)^{2}+s_{4} \frac{\Lambda}{q} \frac{g_{i}}{\theta_{o}} \overline{u_{i} \theta}
\end{aligned}
$$

The first term on the right-hand side is the turbulent production of scale; the second term results in dissipation; the third and fourth terms are diffusion; and the fifth term is a production term which results from buoyancy.

The modeled terms in eqs. (22)-(25) are not expected to contain all possible available information present in the exact third-order correlations they replace. It would be incorrect to believe that the invariant model is more than a careful attempt at modeling the essence of turbulent fluid dynamics. We do not claim to solve the turbulence "exactly," only "accurately." The distinction is very real; the value of any model rests with the ability to give a good description of the turbulent process in a wide variety of applications. That is where the modeling constants come in and where the second half of our "invariant" definition is applied. With a model of this complexity, surely any single flow can be used to determine our constants (disregarding the equations for $\Lambda$ ) such that that one flow is precisely described. However, the chances are high that those same constant values will not be very accurate for a different flow application. But it is our intent to match a number of rather simple flows "well" and thus yield a fixed (or "invariant") set of values for the unknown constants A, b, $s$, and $v_{c}$ that will match all of these flows respectably. "With these constants determined, we can then make predictions in more complicated flows, knowing that our constants will permit a reasonably accurate result. Our success to date verifies this approach (refs. $14,22-24$ ).

The most sensitive model constant is $b$. In a sense, this is good since we must pinpoint the value of $b$ closely or else little will match anything. Analysis of homogeneous turbulence gives values of $b$ between 0.08 and 0.12 . Comparing with other data as well, Donaldson (ref. 16) chose

$$
b=0.125
$$

Computer studies of simple axisymmetric jets and wakes (ref. 24) yield

$$
v_{c}=0.3
$$

The constant A can be shown to be equivalent to the turbulent Prandtl number and, for neutral atmospheric shear layers ( $r e f .25$ ), 
Its value is approximately

$$
A=0.75
$$

By comparing the frequency spectrum of the temperature fluctuations with the spectrum of the turbulent kinetic energy, we find (ref. 22)

$$
s=1.8
$$

The values of the constants of the scale equation are determined by similar examination of the simple flows of axisymmetric jets and wakes, boundary layers, surface layers, grid turbulence, etc., to give (ref. 23)

$$
\begin{aligned}
& s_{1}=-0.35 \\
& s_{2}=-0.6 \\
& s_{3}=0.375 \\
& s_{4}=0.8
\end{aligned}
$$

These constant values have been used to predict many simple flows, including flows not used to set the constants. They have demonstrated the versatility of the invariant scheme and have consistently given us cause for optimism whenever a new flow situation or experimental result permits further verification.

Before proceeding with our model results for vortices, we will outline the numerical procedure used to solve the partial differential equations presented here. Then in Section 5 we discuss the results of our vortex study to date. 


\section{DESCRIPTION OF THE PROGRAM}

The partial differential equations of motion developed in the last section are solved numerically to obtain solutions for vortex interactions and merging. An extensive three-dimensional code was developed through modification and expansion of a basic code previously developed under contract to the Navy for studying submarine wakes (ref. 14). A fairly complete discussion of the numerical technique involved and the operational aspects of the program is contained in references 26 and 27 . Here we wish to summarize the techniques employed in going from the differential equations to the actual solutions they produce.

We first transform the partial differential equations into finite difference form. This step requires decisions regarding the computational grid or mesh and the form of the first and second derivative operators appearing in many places in the complete set of equations. Our code has been written so that it is forwarddifferenced in the marching direction, so that for either $t$ or $\mathrm{x}$, the marching derivative is written typically as

$$
\frac{\partial W}{\partial x} \sim \frac{W_{\text {new }}-W_{\text {old }}}{x_{\text {new }}-x_{\text {old }}}
$$

It is also center-differenced on a uniformly spaced grid in both cross-plane directions, so that for a typical derivative we write

$$
\frac{\partial W}{\partial y} \sim \frac{W_{y}+\Delta y-W_{y}-\Delta y}{2 \Delta y}
$$

where $\Delta y$ is the mesh spacing and $W_{y \pm \Delta y}$ is the value of $W$ at position $y \pm \Delta y$. Second derivative differences are, in fact, derivatives of the first derivative difference. The general finite difference formulation on unequally spaced grids has been presented elsewhere (ref. 28). We merely reduce its complexity by working with uniform meshes. What this reduction does is give us a system that is second-order accurate in the cross plane. This feature yields dividends in solution accuracy. We use a direct solver for the solution of the pressure field (ref. 29) and this particular code requires uniform spacing.

If the equations appear complex. In their differential form, they are much more complicated in finite difference form. Nevertheless, an examination of the equations and our differencing scheme shows that at any point in the computational domain the unknowns there will be related to each other by the coupling of the variables through the production terms and dependent upon values of 
the variable at all four perpendicular points immediately adjacent to the point under observation. Several standard solution techniques are available for solving these equations. Certainly an

explicit scheme exists where we march in $x$ (or $t$ ) by evaluating all of the terms in the equations with the exception of the marching value $\left(e . g \cdot, W_{\text {new }}\right.$ ) with current, old (and, hence, known) values. The difficulty here lies in a restrictive step size $\Delta \mathrm{x}=\mathrm{x}_{n e w}-\mathrm{x}_{0}$ ld that must be imposed to keep the solution scheme stable.

We choose, rather, to use the implicit algorithm applied to two-dimensional cross-plane flows (ref. 30). The scheme works by first linearizing the finite difference equations (of necessity using old values in some places) and then solving for, typically, $W_{\text {new }}$ by assuming that wherever $W$ appears in its individual equation we write $W_{\text {new }}$ (remember, in an explicit scheme, wherever W appears, we almost always write $\left.W_{0} l d\right)$. We then apply a fairly standard algorithm to solve for Wnew and all of the other

variables at $x_{n e w}$. This alternating direction, implicit, tridiagonal algorithm requires the storage of intermediate values; we sweep twice in each direction for each marching step size $\Delta x$ to arrive at $\mathrm{x}_{\text {new }}$ and our solution there.

The linearization is an outgrowth of the refinement of our numerical skills in solving turbulent flows. We break out every equation individually so that the only unknown (and, hence, implicit) variable possible in each equation is the variable for which the equation is solved. Thus, although the $W$ equation contains $U, V$, and $P$, we substitute $W_{o l d}, V_{o l d}$, and $P_{o l d}$ wherever appropriate. In the convection term, we write

$$
\mathrm{w}^{2}=\mathrm{w}_{\mathrm{old}} \mathrm{w}_{\text {new }}
$$

to keep the system linear. We also write the two shear-like terms as

$$
\begin{aligned}
& \overline{\mathrm{VV}}=-\varepsilon_{\mathrm{y}} \frac{\partial W}{\partial \mathrm{y}}+\mathrm{C}_{\mathrm{VV}} \\
& \overline{\mathrm{VW}}=-\varepsilon_{\mathrm{z}} \frac{\partial W}{\partial \mathrm{z}}+\mathrm{C}_{\mathrm{VW}}
\end{aligned}
$$

with ${ }^{\varepsilon_{\mathrm{y}}}, \varepsilon_{\mathrm{W}} \varepsilon_{\mathrm{z}}, \mathrm{C}_{\mathrm{VV}}$, and $\mathrm{C}_{\mathrm{vW}}$ evaluated at ( )old ${ }$, The rest before application of the algorithm

The set of finite difference equations is solved in a computer program called WAKEX operational at the NASA Langley CDC 6600 computer facility. The User Manual pertaining to program operation is in reference 26. Here we summarize the technique used to initialize a computation; the next section of this report gives results for a variety of vortex flows which have been studied. 
The code has been developed to simulate the merging and interaction of a trailed vorticity from a wing. With a given load distribution, a vortex sheet is trailed from the trailing edge of the wing. In principle, the velocity field associated with this trailed sheet could be input as an initial condition to WAKEX. Unfortunately, an accurate numerical resolution of this flow field would require a computational grid with a prohibitively large number of grid points. Rather, we input a Gaussian of vorticity to represent each vortex in the wake. We have chosen to do this rather than use the velocity distributions given by the Betz model for two reasons. The first is that the Betz swirl velocity profiles are inviscid and, therefore, do not have a viscous core. If these profiles were input as initial conditions, many grid points would have to be used to resolve the high swirling velocities. The second reason is that much computer time would be used simply to adjust the inviscid profiles to their viscous counterparts. The Gaussian distribution of vorticity we use is chosen to have the same circulation centroid and dispersion as the vortex it replaces.

As an example, the initial conditions which would be used to compute the decay of a linearly loaded wing are now given. If the load distribution on a wing of semi-span $s$ is given by $\Gamma=\Gamma_{0}(I-|y| / s)$ for $-s \leq y \leq s$, the centroids of the two counter-rotating vortices are $\bar{a} t \bar{y}= \pm s / 2$. The dispersion of the trailed vorticity is computed from

$$
r_{c}^{2}=\frac{\int_{0}^{s}(n-\bar{y})^{2} \frac{d \Gamma}{d \eta} d \eta}{\int_{0}^{s} \frac{d \Gamma}{d \eta} d \eta}
$$

and, for the linearly loaded wing, is $r_{c} \cong 0.3 \mathrm{~s}$. A positive vortex of strength $\Gamma_{0}$ is positioned at $\bar{y}=0.5 \mathrm{~s}$ with a swirling velocity distribution given by

$$
V=\frac{\Gamma_{0}}{2 \pi r}\left\{1-\exp \left[-12\left(\frac{r}{s}\right)^{2}\right]\right\}
$$

where $r^{2}=(y-\bar{y})^{2}+(z-\bar{z})^{2}$. The image vortex at $\bar{y}=-0.5 s$ is automatically accounted for through reflection boundary conditions at $\mathrm{y}=0$.

For an "interior" or flap vortex, the dispersion formula is modified as shown in reference 2 to read 


$$
r_{c}^{2}=\frac{\int_{y_{A}}^{y_{B}}\left(n-\bar{y}_{A B}\right)^{2} \frac{d \Gamma}{d \eta} d n}{\int_{y_{A}}^{y_{B}} \frac{d \Gamma}{d \eta} d \eta}
$$

and the interior vortex is positioned at its appropriate centroid.

Because of computer core size and execution time costs, we have developed the program to permit no more than four such Gaussians in the computational grid, which itself can be no larger than $65 \times 65$ points. When an image plane exists (say, the other half of the wing), the program is organized to solve for the righthand half of the flow and provide appropriate matching/reflection conditions at $\mathrm{y}=0$ to handle the image vortices present in the left-hand plane. The initialization procedure involves telling WAKEX where at $\left(y_{c}, z_{c}\right)$ the vortices of strength $\Gamma_{c}$ and spread $r_{c}$ are located. We have found that typically 5 grid points are needed in the e-folding of the Gaussian to give reasonable results. This would fix the computational grid spacing at about $0.05 \mathrm{~s}$ for the above-noted example.

It makes little sense to extend the computational domain far beyond the region of interest, yet we cannot set $V=W=0$ along such boundaries, since every vortex pair has a dipole far-field and every isolated vortex has a swirling velocity which decays as $\mathrm{r}^{-1}$. We know, however, that well outside the region of vorticity, the vortex-induced velocities should behave, to lowest order, as $\mathrm{V} \propto \mathrm{z} / \mathrm{r}^{2}$ and $\mathrm{W} \propto \mathrm{y} / \mathrm{r}^{2}$. These expressions are appropriate for a single vortex but are also just the first terms in a far-field expansion of the moments of vorticity distribution in the computational domain. We have carried out the moment expansion to order 2 where

$$
I_{n m}=\iint \zeta y^{m} z^{n} d y d z ; n, m \leq 2
$$

and have included the appropriate $I_{n m}$ factors in the far-field boundary conditions on $V$ and $W$. This analysis is summarized in Appendix D. By invoking this option in the program, we permit any accurate vortex representation to serve as velocity initial conditions in WAKEX.

In the spirit of specifying equivalent Gaussians of vorticity, we permit initialization of temperature (or concentration) $\theta$, axial velocity $U$, and turbulent kinetic energy $q^{2}$ by

$$
\text { Value }=\text { Maximum } \exp \left(-r^{2} / r_{c}^{2}\right)
$$


where $r$ and $r$ carry the same definitions as before. Thus, for any set (of up to four each) of $\theta$, $U$, or. $q^{2}$, a specification of maximum value, spread rate, and location $\left(y_{c}, z_{c}\right)$ of the center will permit initialization of these quantities.

Having specified the initial conditions on the appropriate dependent variables, the initialization is complete except for program execution configuration and printout specifications. These are covered in the User Manual. Perhaps, before summarizing the results of our study, 1t would be best to caution the reader regarding program performance. Even though we have had a surprisingly high success rate, that rate cannot be duplicated by others without following some rather basic rules. Perhaps all of the caution can be contained in the warning that WAKEX will not return an accurate solution except when the initialization procedure gives an accurate, compatible, and acceptable set of profiles. Enough points must be present in the computational grid to permit the complete equations to keep an accurate track of solution behavior. The boundaries of the computational domain cannot be so close to the region of variability (the $y_{c}, z_{c}$ values) that they give a rapid jump to the far-field conditions. Nor can the solution scheme be driven too fast in its simulation. We are generally restricted to permitting about $5 \%$ change in the variables in the marching direction, while cross-plane velocities and step sizes must be compatible with the courant condition

$$
\frac{V \Delta t}{\Delta \mathrm{y}} \leq I ; \frac{W \Delta t}{\Delta \mathrm{z}} \leq I
$$

which does not permit the solution to march faster than it takes information to convect from one mesh point to its neighbor. The initial stages of vortex roll-up and interaction are convective in nature and demand that these criteria be followed. Once turbulent diffusion begins dominating the flow, the nearness of the boundaries will generally decide the overall time of the computer simulation.

In the next section of this report, we use the Vortex Wake program to follow the behavior of various configurations with and without image planes for vortices, turbulent energy, axial velocity, and concentration initialized in ways to give an overall view of the phenomena of vortex merging and interaction. Our examples do not cover the full breadth of cases possible (nor, in fact, cases we have run) but are used to illustrate the power of the turbulent code in simulating vortex dynamics and to suggest the minimum hazard vortex wake configuration. 


\section{COMPUTATIONS USING THE "WAKE VORTEX" TRANSPORT CODE}

In this section, a variety of computations are presented to demonstrate the flexibility of the Wake Vortex transport code. Results of numerical merging studies have identified a low hazard vortex wake, and these findings are presented in section 5.2 .

\subsection{Decay of a Vortex Pair}

In a calm, neutral atmosphere.- The simplest vortex wake is that generated by an aircraft in cruise configuration. This wake is one which can be adequately described by a simple vcrtex pair. Figure 22 shows the initial conditions for the computation. The initial vorticity and turbulent kinetic energy distributions were taken to be Gaussian about $\mathrm{y} / \mathrm{s}^{\prime}= \pm 1$. Two computations were made with the viscous core radius $r_{c} / s^{\prime}=0.4$ and 0.8 . The integral scale parameter $\Lambda / s^{\prime}$ was taken equal to 0.2 . The turbulent energy components were equally distributed with $\overline{u u}=\overline{v v}=\overline{w w}=$ $\mathrm{q}^{2} / 3$. The cross-correlations were initially taken to be zero and $\Gamma / \nu=10^{4}$.

The computations were carried out in time and the results transformed to downstream distance through $\mathrm{x}=\mathrm{U}_{\infty} \mathrm{t}$. Figure 23 shows the circulation computed over the area $y \geq \infty$. The nearly constant circulation region for the initially tight vortex $\left(r_{c} / s^{\prime}=0.4\right)$ illustrates that a time interval exists before the diffusion of vorticity across the wake centerline is significant. This time period is a measure of how long a point vortex simulation of the pair could be expected to be valid.

The computation for the initially more diffuse vortex $\left(r_{c} / s^{\prime}=0.8\right)$ was carried downstream to a distance of $x^{\prime} / \mathrm{b}^{\prime}=$ $8 \pi \mathrm{Ab} / 2 / \mathrm{b}^{2} \mathrm{C}_{\mathrm{L}}$; at $\mathrm{C}_{\mathrm{L}}=1$ and $\mathrm{A}=7$ and a rectangularly loaded wing, $x / b \simeq 160$. For the $B-747$ aircraft, this is approximately $10 \mathrm{~km}$. Without ambient atmospheric turbulence, the vortex pair ages quite slowly. Figure 24 compares the maximum root-mean-square turbulence level $\mathrm{q}$ between the pair and a computation using a single isolated vortex having the same initial conditions as one vortex of the pair. The level of turbulence intensity in the pair is not significantly different from that computed for an isolated vortex.

In figure 25. we show the $r_{c} / s^{\prime}=0.8$ circulation as a function of downstream distance about a square of side $2 \mathrm{e}$, centered at the centroid of vorticity in the half-plane. As might be expected, the decay of circulation is far more rapid than the half-plane value.

Figure 26 shows the computed descent rate vertical centroid $\bar{z}$ of the vorticity. The $\bar{z}$ $\mathrm{dh} / \mathrm{dt}$ of the increases as a 

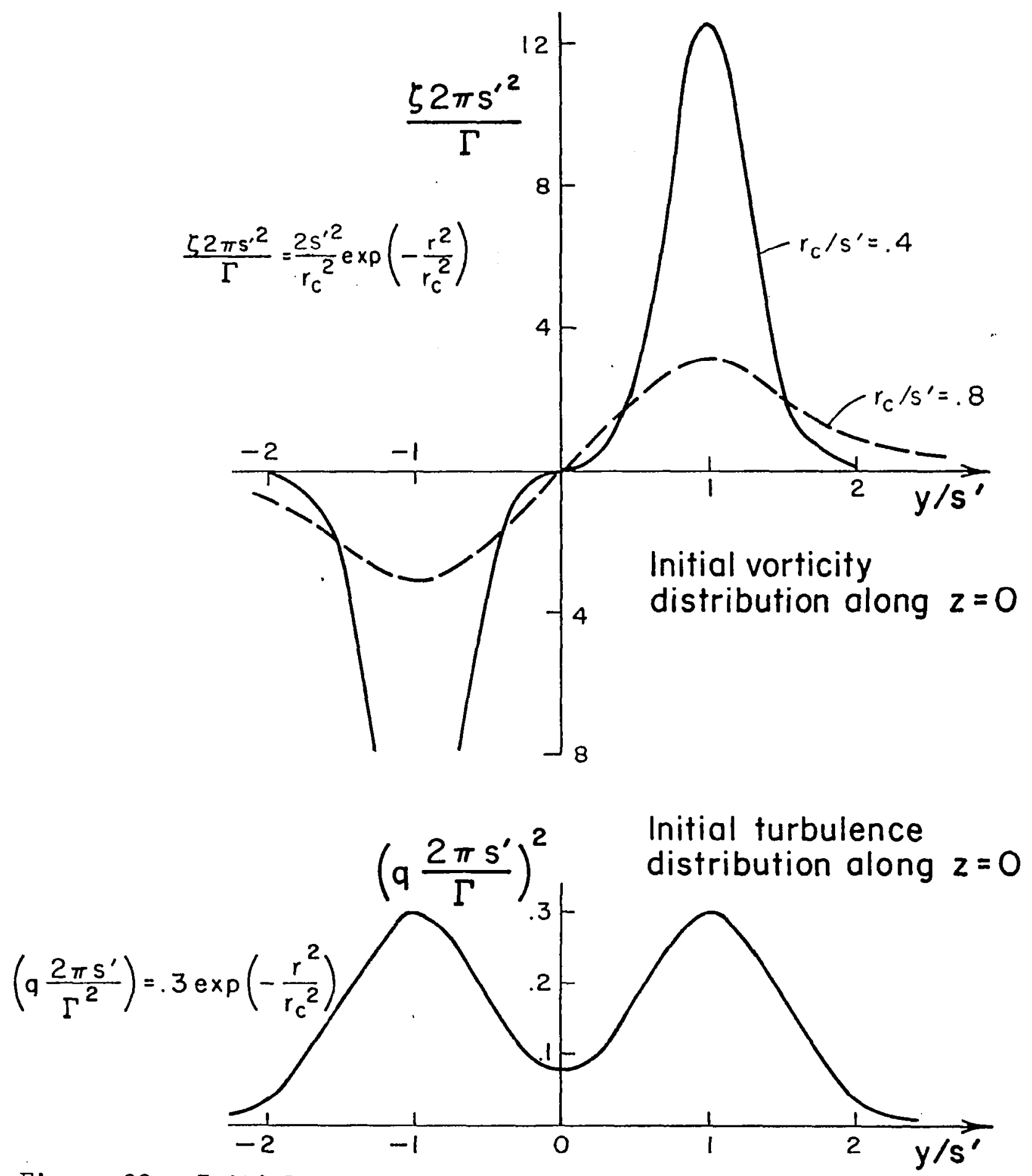

Figure 22. Initial vorticity and turbulent kinetic energy distributions used in the computation of the decay of a vortex pair in a quiescent atmosphere. 


\section{Half-Plane Circulation}

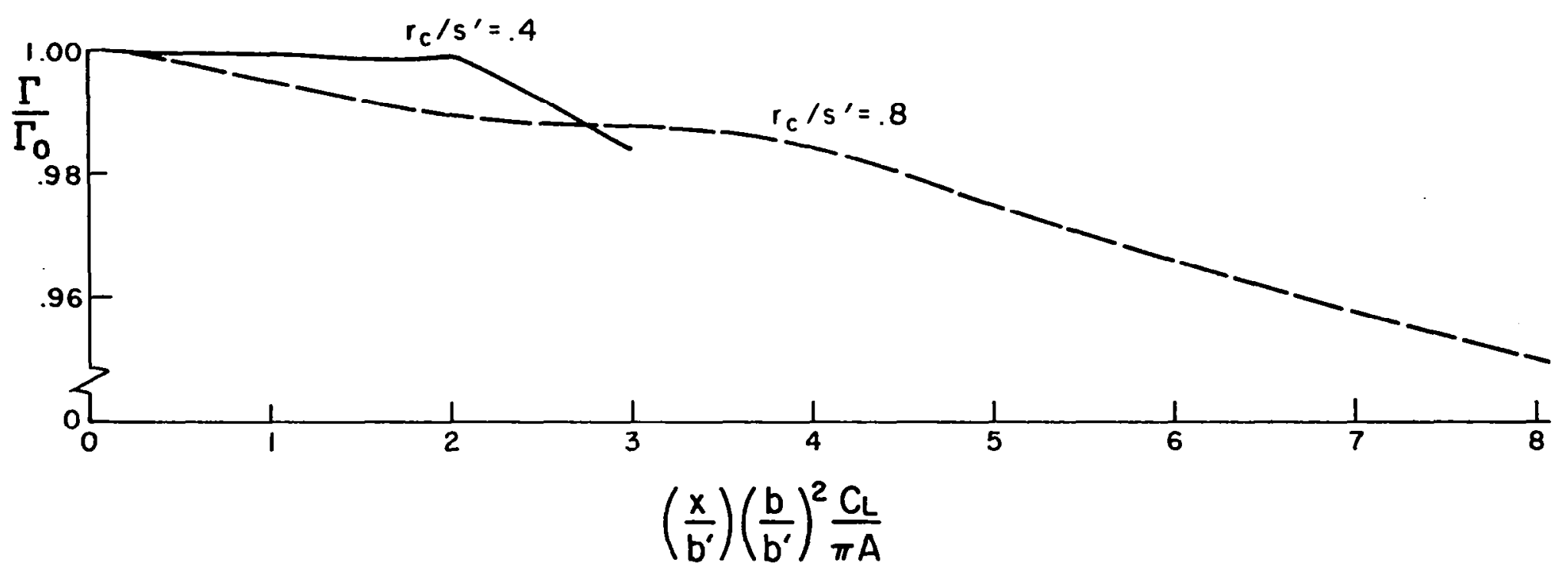

Figure 23. Comparison of the decay of circulation in a counter-rotating vortex pair for two different initial vortex core radil. 


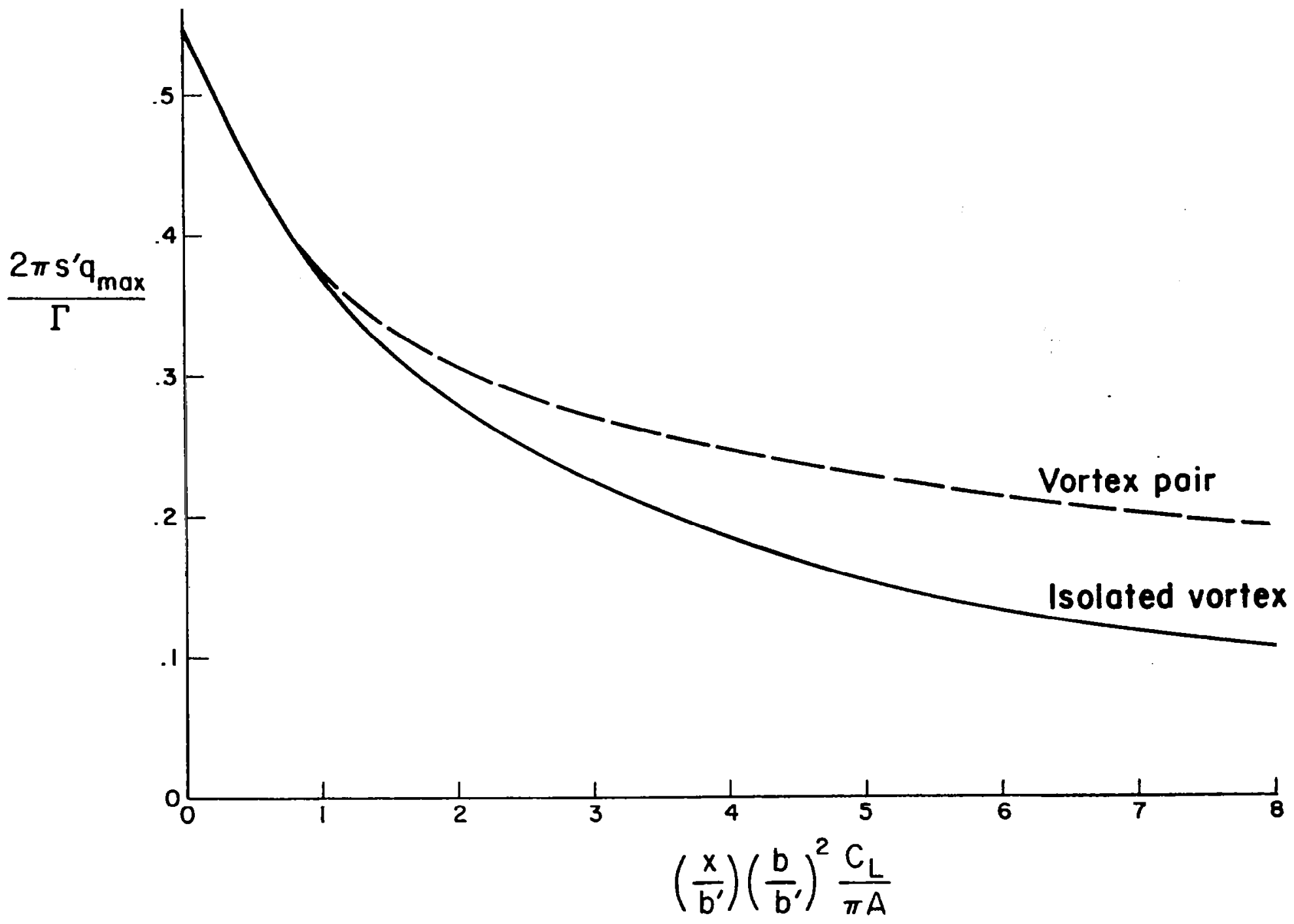

Figure 24. Comparison of the maximum root-mean-square turbulence level in a counter-rotating vortex pair and in an equivalent isolated vortex $\left(r_{c} / s^{\prime}=0.8\right)$. 
$\underset{f}{M}$

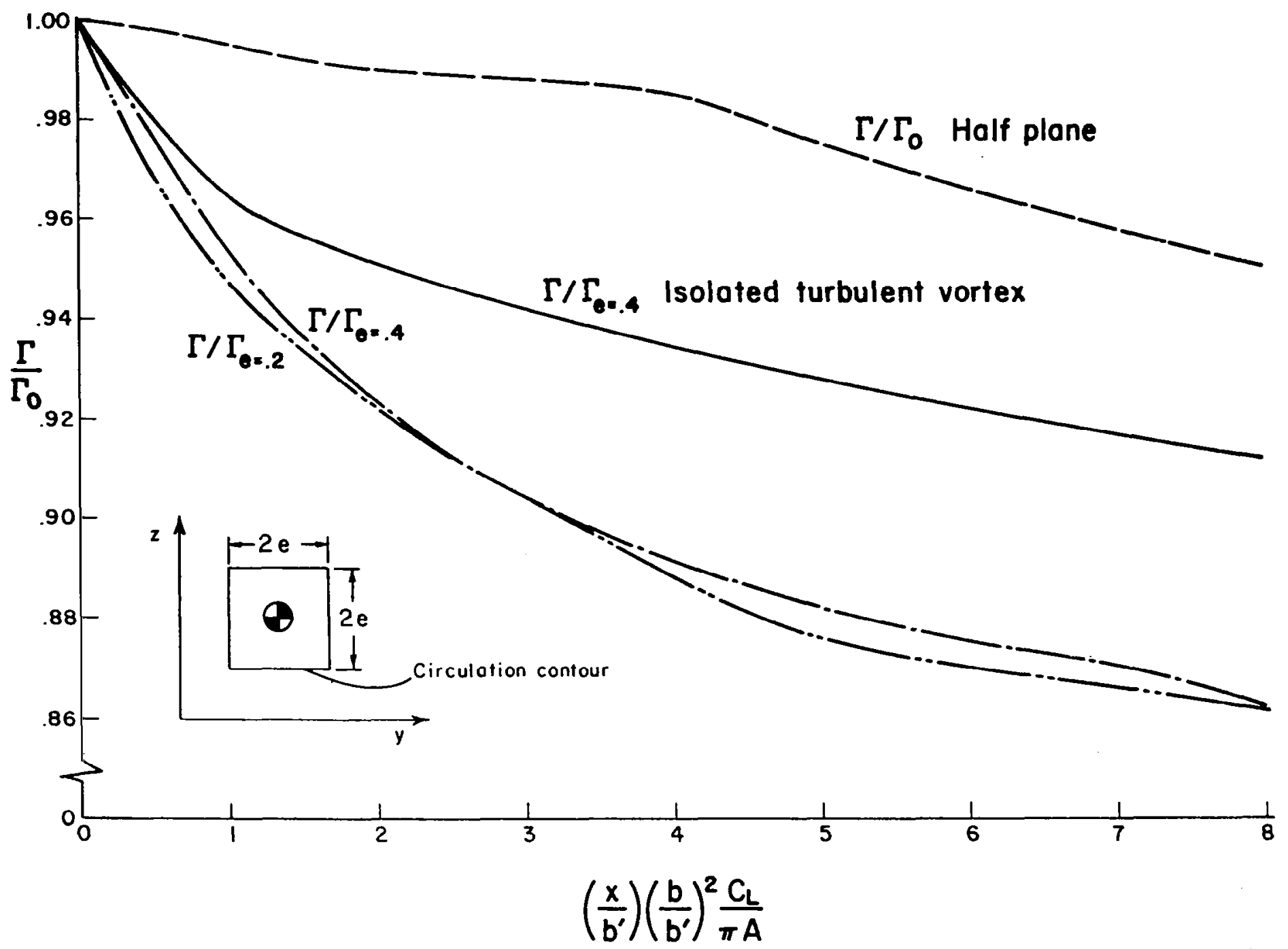

Figure 25. Decay of circulation in a counter-rotating vortex pair computed about square contours centered at the centrold of vorticity of the half-plane vorticity distribution $\left(\mathrm{r}_{\mathrm{c}} / \mathrm{s}^{\prime}=0.8\right)$. 


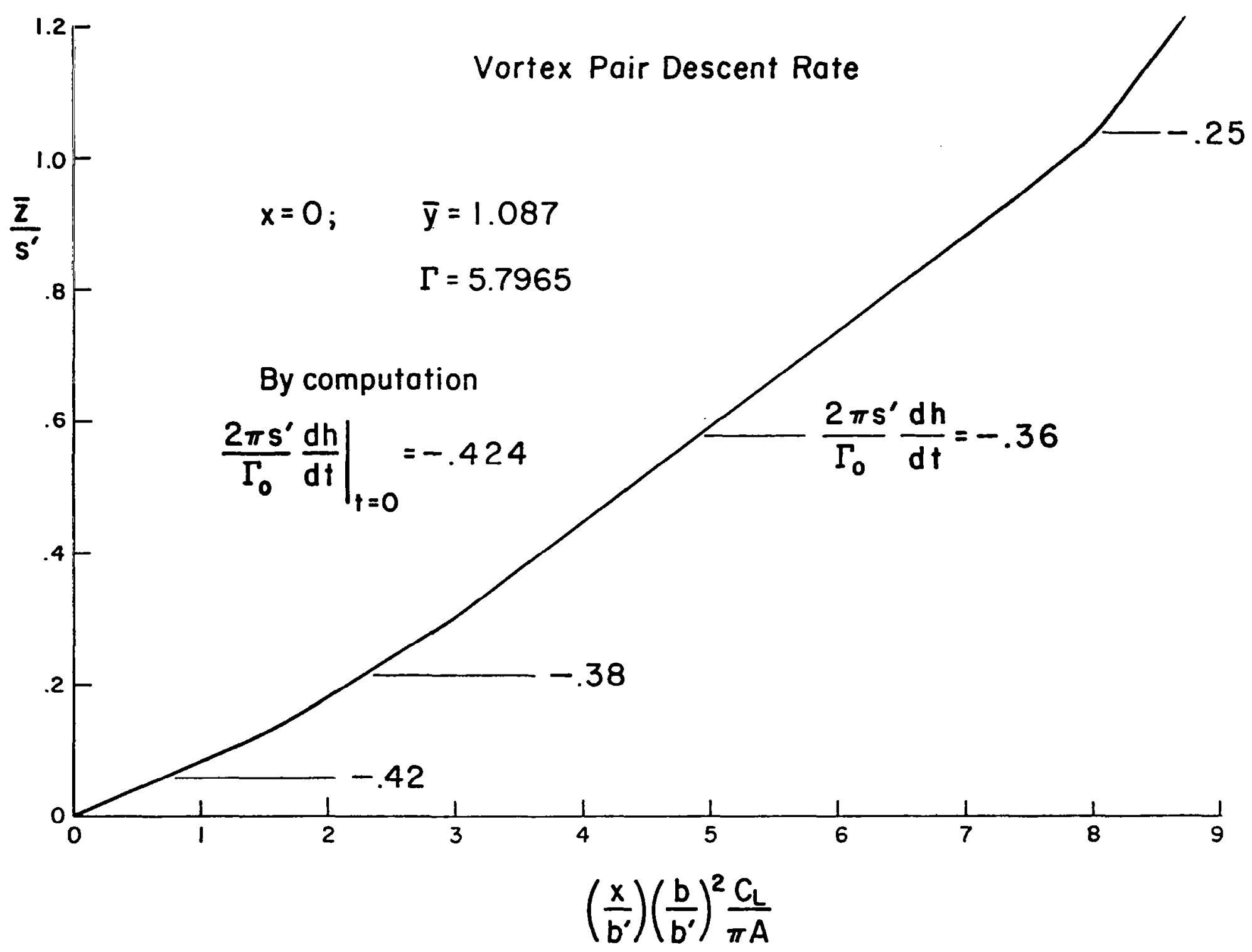

Figure 26. Descent rate of the counter-rotating vortex pair $\left(r_{c} / s^{\prime}=0.8\right)$. 
function of downstream distance since a uniform upwash has been added to the computation (of magnitude $\Gamma_{o} / 4 \pi s^{\prime}$ ) to keep the vortex pair centered in the computational mesh. The descent rate decreases as vorticity diffuses across the aircraft centerline.

In figures 27 and 28 are shown instantaneous streamlines at the beginning and end of the computation for the $r_{c} / s^{\prime}=0.8$ computation. The $\mathrm{y}, \mathrm{z}$ coordinate system is one in which the fluid at infinity is at rest; therefore, the pair descends downward.

Wake dissipation by atmospheric turbulent diffusion of vorticity.- There has been an attempt (ref. 3l) to correlate wake lifetime with the turbulent dissipation rate $\varepsilon$ (our model at high Reynolds number gives $\varepsilon=0.125 \mathrm{q}^{3} / \Lambda$ ). Vortices present their greatest hazard under stable atmospheric conditions. Under these conditions, scales can be smaller than the characteristic wake length scale--the vortex spacing. Under these problem atmospheric conditions, the turbulent dissipation rate does not contain sufficient information to estimate the diffusion of trailed vorticity in the atmosphere. The integral or macroscale must be known. This fact can be demonstrated by two computations in which $\varepsilon$ is held constant. For light and light-to-moderate turbulence, we use a value of $\varepsilon^{1 / 3}=2 \mathrm{~cm}^{2 / 3} / \mathrm{sec}$ as suggested in reference $3 I$.

If we take $\Lambda / s=0.2$ and 2.0 , the corresponding turbulent kinetic energies become $(2 \pi \mathrm{sq} / \Gamma)^{2}=0.025$ and 0.116 , respectively. These two values can probably be achieved in a stable atmosphere with conventional jetliners.

The initial conditions are again Gaussians of vorticity at $y / s= \pm 1$ of opposite sign to produce a counter-rotating pair. The dispersion radius is taken to be $r_{c} / s=0.5$. The turbulent energy components are again equally distributed so that $\overline{u u}=\overline{v v}=\overline{W w}=$ $\mathrm{q}^{2} / 3$ initially. Integral scale parameter and turbulent energy components are held constant on the computational boundaries and $\Gamma / \nu=10^{4}$.

Figure 29 shows the circulation decay computed about a box contour of side $2 e$ centered at the centroid of the vorticity. At the end of the computation (typically a wake age of $60 \mathrm{sec}$ ), levels differ by a factor of 2. More important, the rate of decay differs by approximately a factor of 4 . These results do well to emphasize the importance of scale and caution against too simple a parameterization of the atmosphere. Obviously, the effects of wind shear and stratification help to make the analysis of the interaction of a vortex wake with the atmosphere a most difficult one.

An indirect comparison can be made between the above results and figure 25 which gives circulation decay of a vortex pair in a quiescent atmosphere. The initial spread of vorticity is different in that $r_{c} / s=0.8$ for the pair in the quiescent atmosphere, while $r_{c} / s=0.5$ for the above cases. The abscissas on figures 


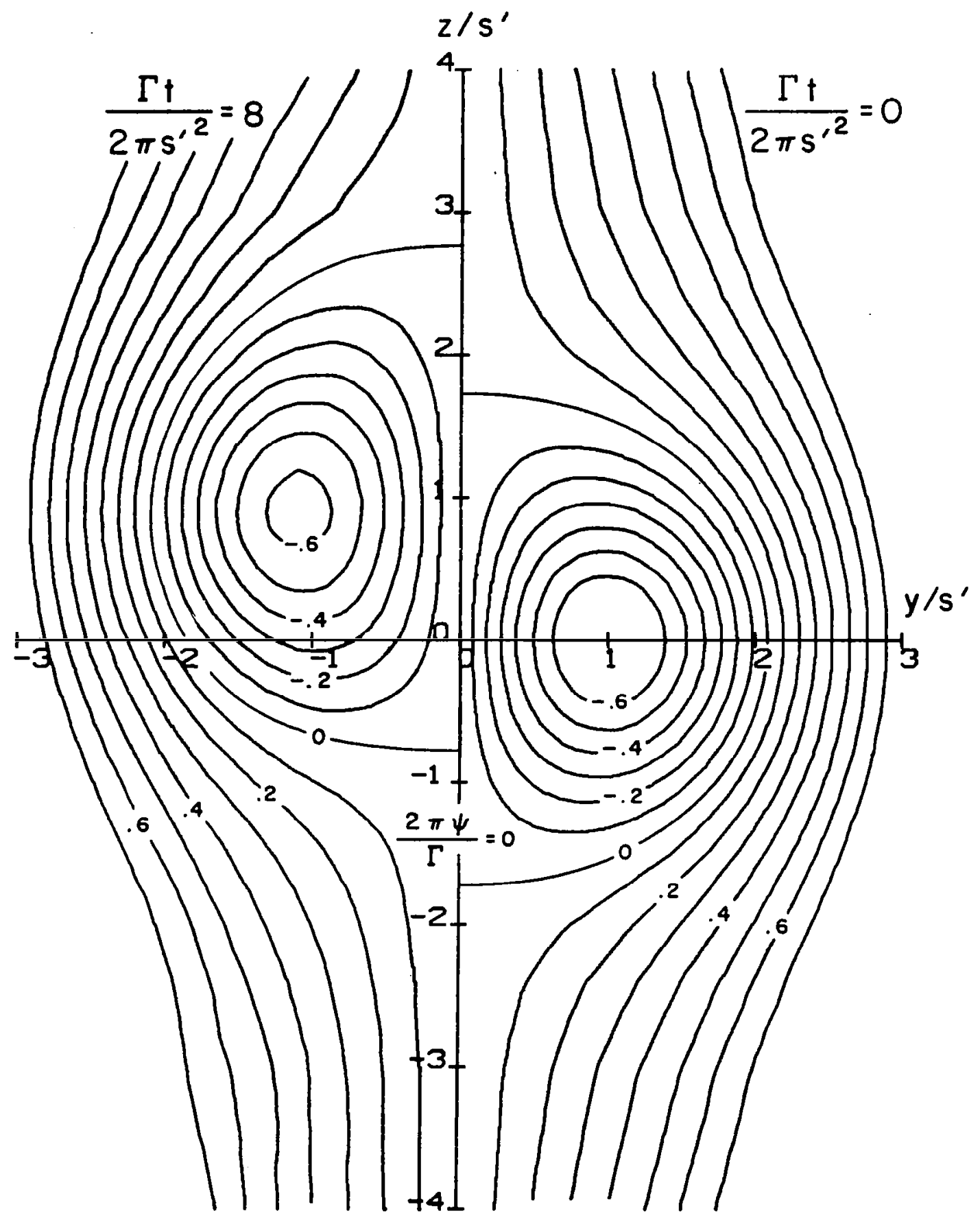

Figure 27. Instantaneous streamlines at $\Gamma t / 2 \pi s^{\prime 2}=0.0$ and 8.0 . The descent of the pair is stopped by adding an upwash of $\Gamma / 4 \pi s^{\prime}$. Streamilnes shown for $\Gamma t / 2 \pi s^{\prime 2}=8.0$ have been reflected across the $y=0$ axis. 


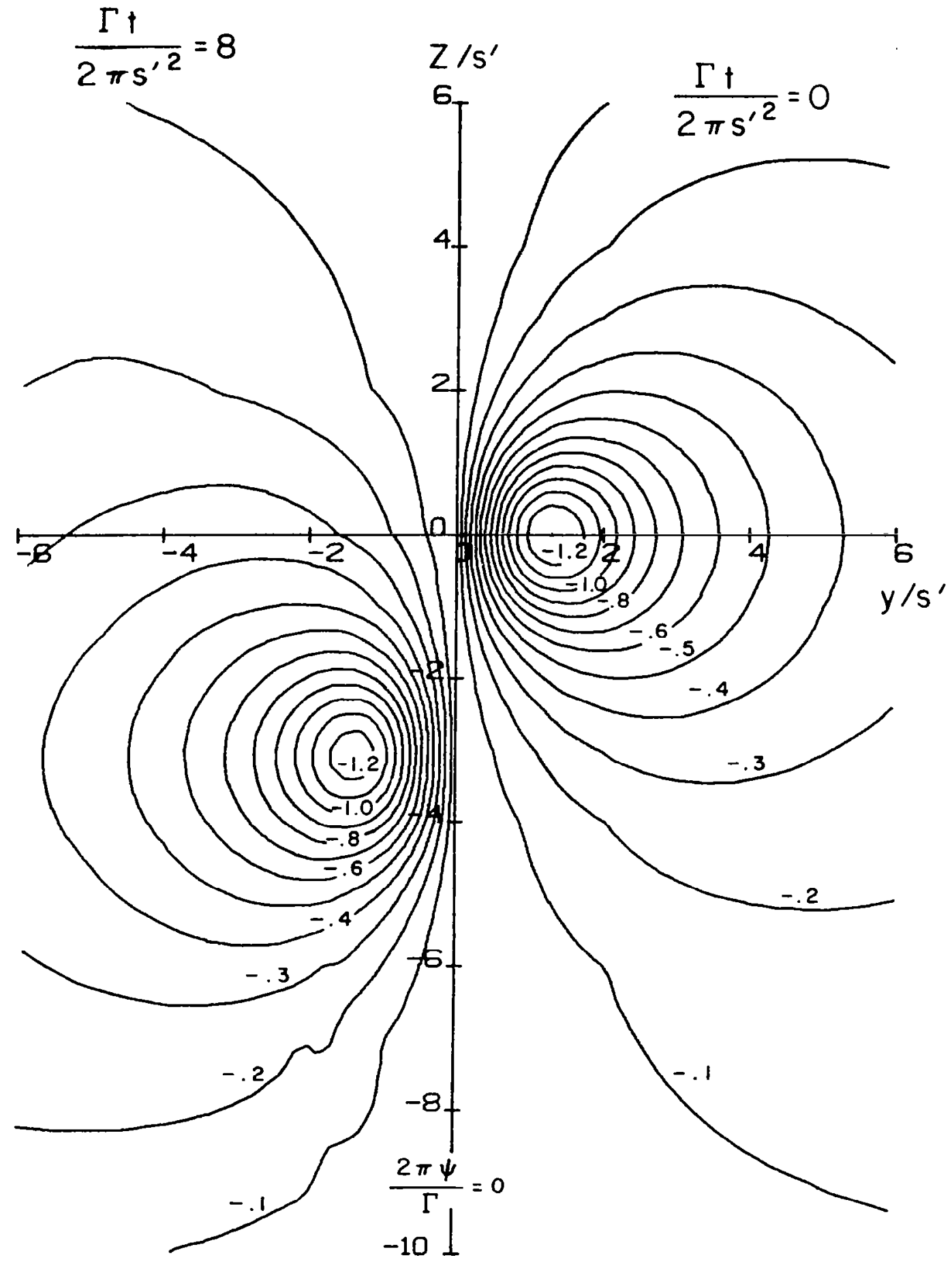

Figure 28. Instantaneous streamlines at $\Gamma t / 2 \pi s^{, 2}=0.0$ and 8.0 . The upwash added in Figure 27 has been removed and the pair permitted to descend. Streamlines shown for $\Gamma t / 2 \pi s^{\prime}=8.0$ have been reflected across the $y=0$ axis. 


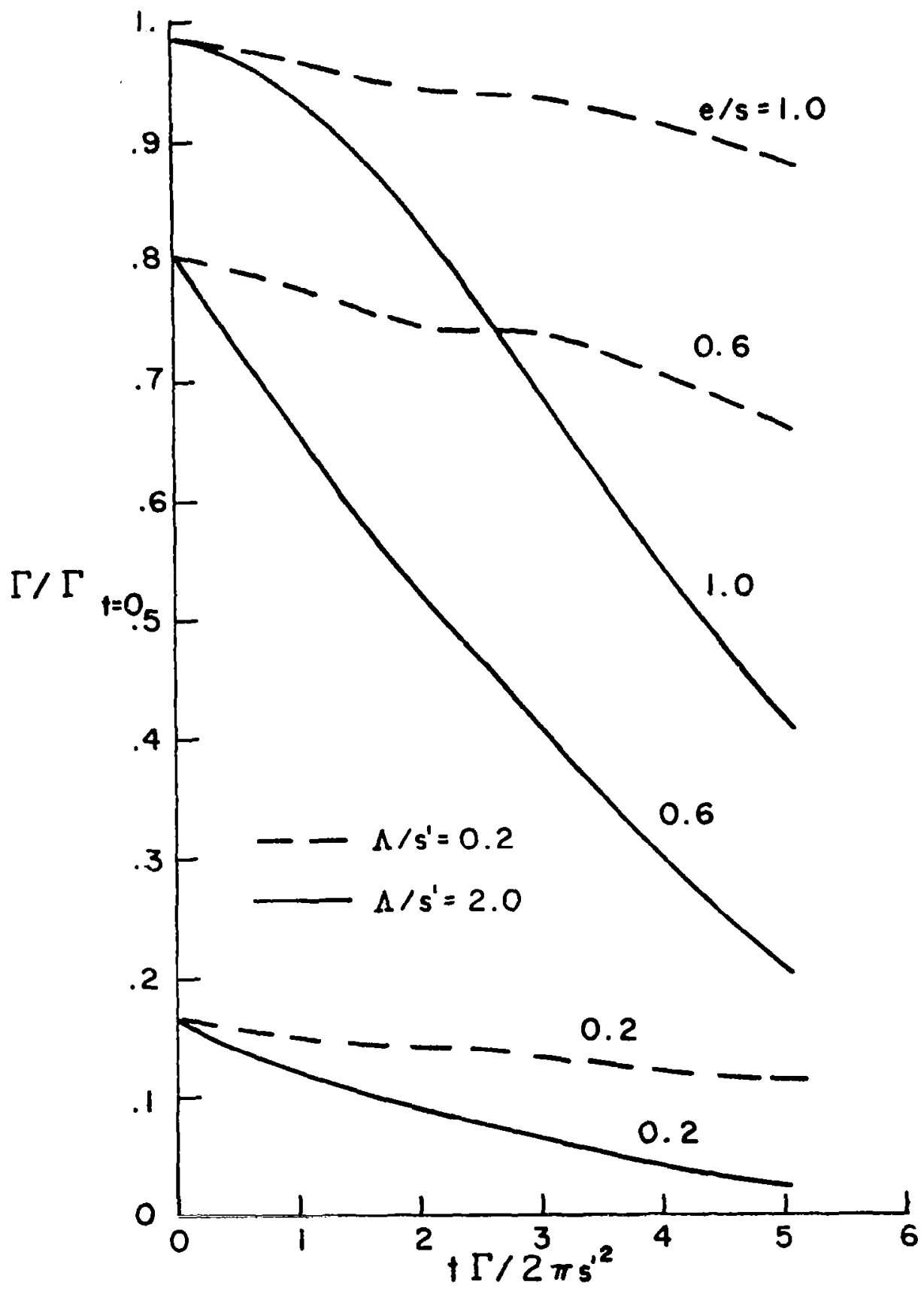

Figure 29. Decay of circulation in a counter-rotating vortex pair 1mmersed in a turbulent atmosphere with constant turbulent dissipation rate $\left(\varepsilon^{1 / 3}=2 \mathrm{~cm}^{2 / 3} / \mathrm{sec}\right)$; see figure 25 . 
24 and 29 are equal, and so time or downstream positions can be compared directly. The slow decay of the pair in a quiescent atmosphere is clearly demonstrated. The dominance of the atmosphere in controlling the ultimate death of a vortex wake is absolute even for low ambient turbulence levels. The turbulent atmosphere may be likened to an infinite reservoir of turbulent kinetic energy which can, for unlimited time, nibble away at the vortex wake.

Work is underway to investigate through second-order closure turbulent modeling the fluid dynamics of the lower atmosphere. This work (partially reported in ref. 32) should give the tools necessary to access the rate of wake aging which results from interaction with a dynamic atmosphere.

\subsection{Turbulent Merging}

The result of vortex merging is the redistribution of vorticity by convection and turbulent diffusion. In this subsection, model calculations of merging are undertaken to demonstrate the phenomenon qualitatively and quantitatively. These results are then used to identify a minimum hazard vortex wake.

Merging computations.- An isolated vortex has streamlines which are circles centered on the vortex axes. The only mechanism which can redistribute vorticity radially outward is diffusion, both laminar and turbulent. It is well known that the laminar aging of a vortex is an extremely slow process. Unfortunately, the axisymmetric vortex is quite stable and can only sustain low turbulence levels. In fact, turbulence is actually damped in the central region of the vortex (the viscous core). It is just in this region where vorticity is a maximum, and the only turbulent redistribution of vorticity in this region results from turbulence which has diffused into the core.

That vortex cores from simply loaded wings are, in fact, nearly laminar can be seen in figure 5 of reference 33 where smoke released from a tower has been entrained into the wing tip vortex of a large aircraft. The air through which the aircraft flies is discernibly turbulent (as interpreted from the dispersion of smoke) while the vortical fluid making up the viscous core is nearly laminar.

Turbulent computations using second-order closure modeling also confirm this feature of isolated vortices. Taking the equilibrium, nondiffusive, and high Reynolds number limits of the secondorder closure model (ref. 2), we obtain the turbulent kinetic energy distribution shown in figure 30 Also shown in the corresponding distribution of vorticity. Note that no turbulent kinetic energy is predicted for $r / r_{c} \leq 1.25$. The centrifugal effect of the swirling velocity suppresses the production of turbulence in this region. 


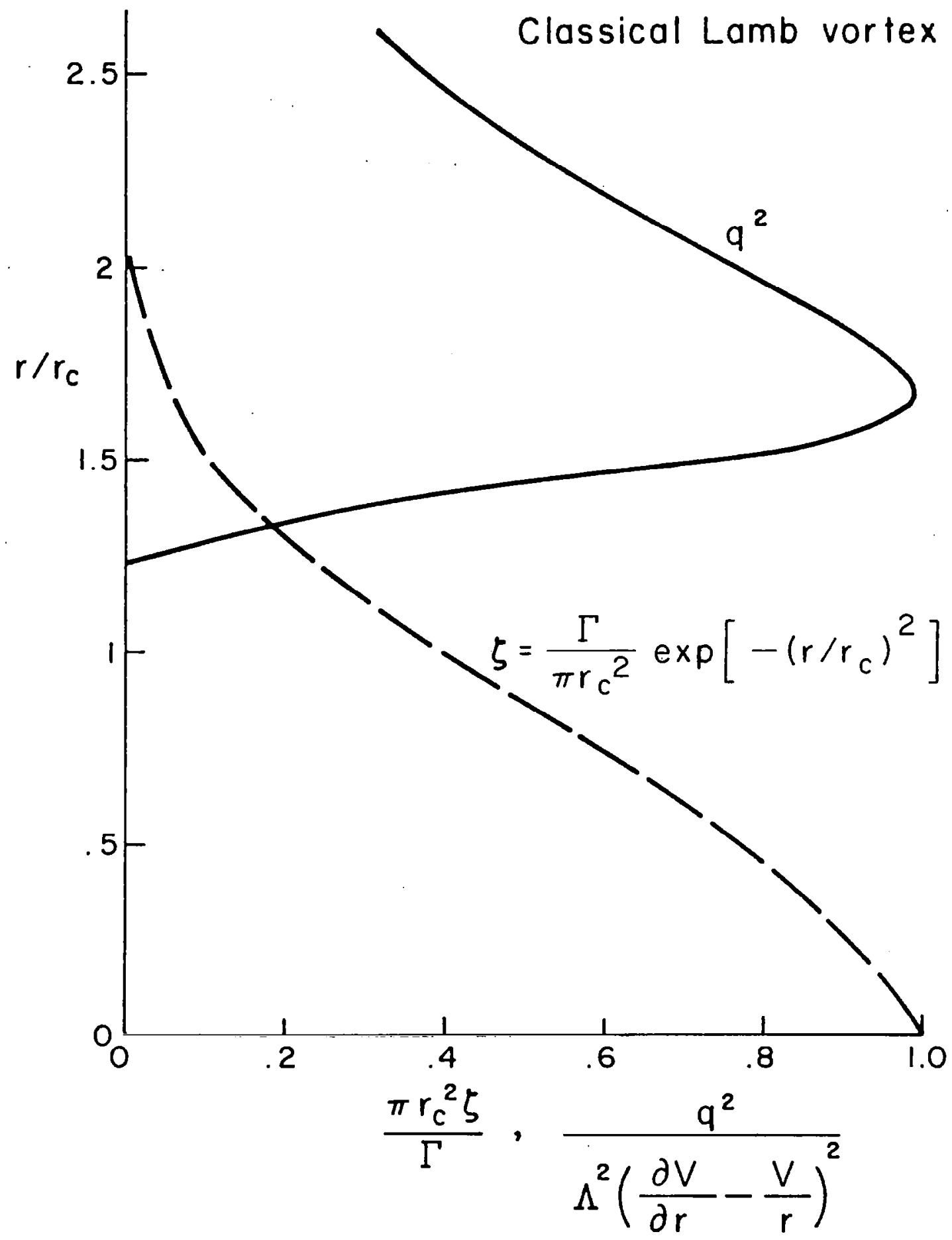

Figure 30. The superequilibrium turbulent kinetic energy and vorticity distribution in a Lamb vortex 
This discussion has neglected the axial velocity and, hence, shear as an additional source of turbulence. However, from observations and analytic considerations (refs. 2, 34), aircraft vortices do not have sufficient axial shear to provide a mechanism for any sizable production of turbulence.

The presence of two or more vortices in proximity destroys the axial symmetry of a vortex by an induced straining field. Once the symmetry has been destroyed, turbulence can be produced which leads to the subsequent diffusion of vorticity. As a first calculation of the merging phenomenon, take the merging problem between two like-sign, equal strength vortices. Two Gausian spots of vorticity of the form

$$
\frac{\pi d^{2} \zeta}{2 \Gamma}=\frac{d^{2}}{2 r_{c}^{2}} \exp \left(-\frac{r^{2}}{r_{c}^{2}}\right)
$$

have been placed at $y= \pm d / 2, z=0$. The radius $r$ is measured outward from both $y= \pm d / 2, z=0$. Gaussians of turbulent kinetic energy of the form

$$
q^{2}=q_{0}^{2} \exp \left(-\frac{r^{2}}{r_{c}^{2}}\right)
$$

are also placed at $y= \pm d / 2, z=0$ with

$$
q_{0}^{2}=0.01(\Gamma / \pi d)^{2}
$$

The calculation is started with $\overline{u u}=\overline{v v}=\overline{w w}=q^{2} / 3$ and $\overline{u v}=0$. The integral scale parameter is initially taken equal to $0.1 d$. Boundary conditions on velocity are applied via a multipole expansion of the vorticity field (Appendix D). Turbulent quantities are set equal to zero at the computational boundaries.

The results of the computations are shown in figures 31,32 , and 33 where instantaneous distributions of pressure, vorticity, and turbulent kinetic energy are shown in intensity form. The counter-clockwise rotation of the pair is expected. It is interesting to note that the mean flow variables, pressure and vorticity, take on a discernibly more axisymmetric structure at $t \Gamma / \pi d^{2}=4$ than the turbulent kinetic energy. This is expected in a phenomenon in which the redistribution of vorticity is initially governed by convection. The initial time scale for turbulent redistribution through diffusion is of the $\theta(\Lambda / q)$ which is approximately $t \Gamma / \pi d^{2}=1$. 

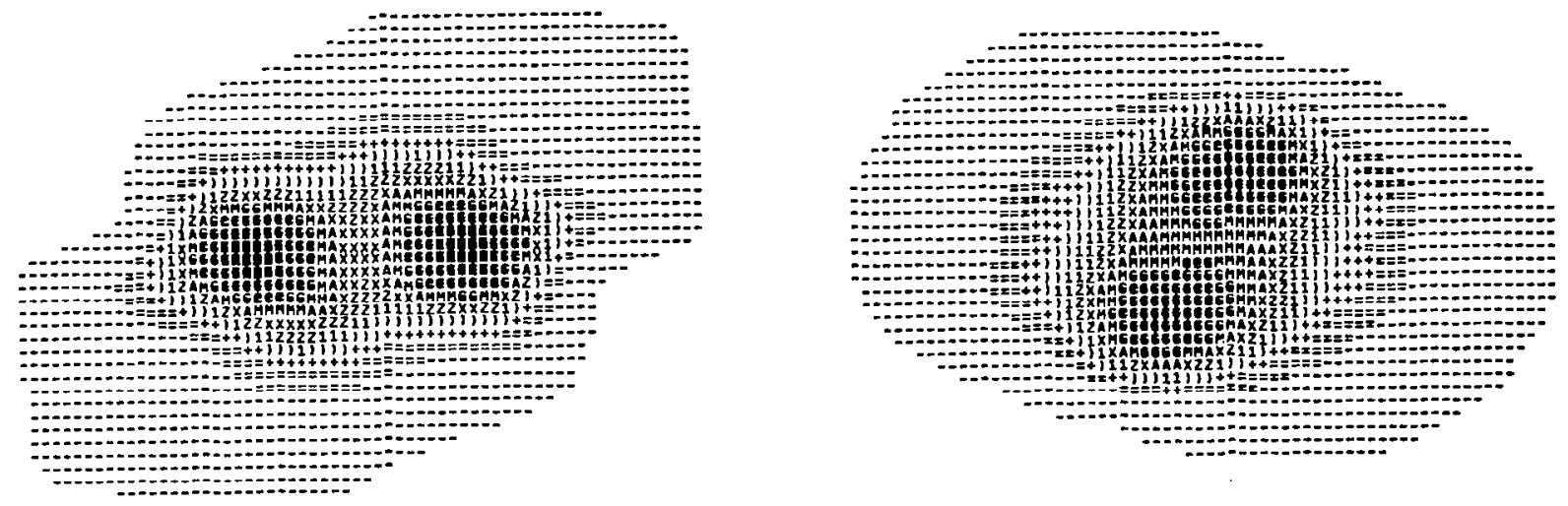

$t \Gamma / \pi d^{2}=0.0 ; P_{m i n} / P_{n}=1.0$

$t \Gamma / \pi d^{2}=1.33 ; P_{m 1 n} / P_{n}=0.75$
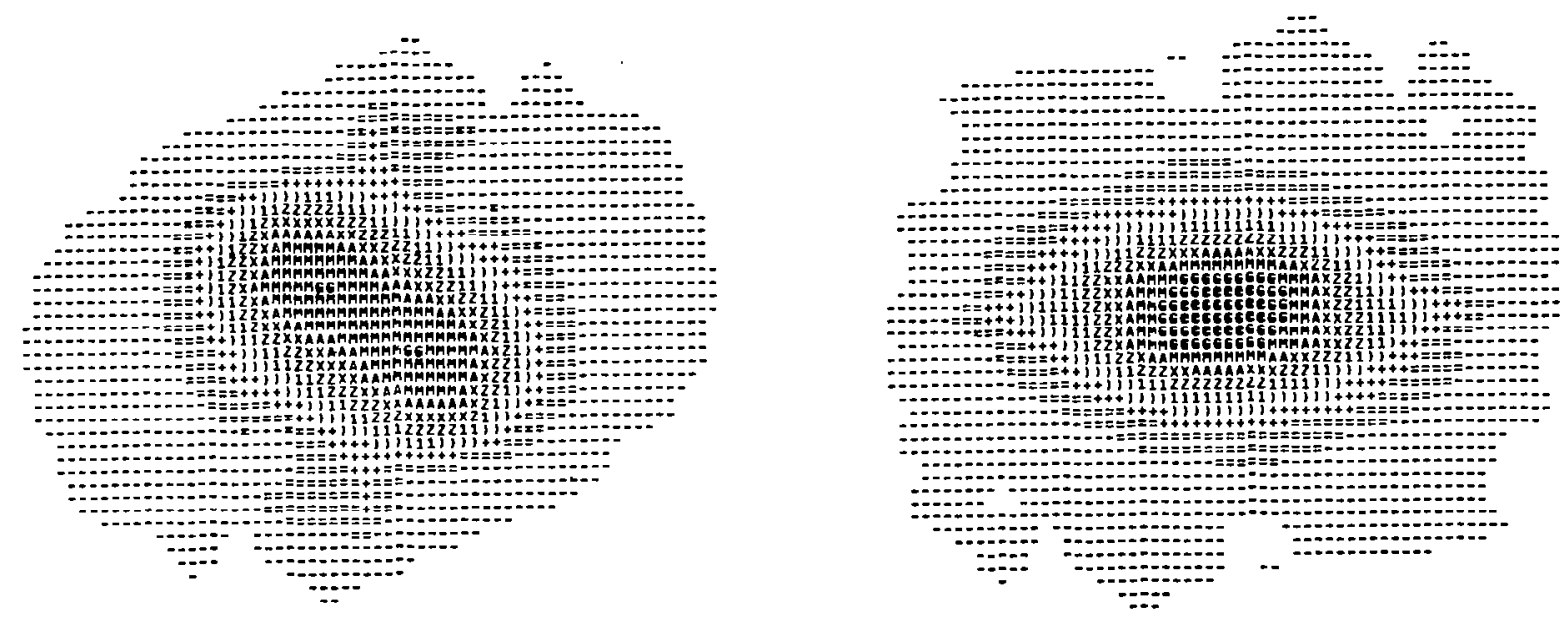

$\mathrm{tr} / \pi \mathrm{d}^{2}=2.67 ; \mathrm{P}_{\mathrm{min}} / \mathrm{P}_{\mathrm{n}}=0.52$

$t \Gamma / \pi d^{2}=4.0 ; P_{m 1 n} / P_{n}=0.65$

F1gure 31. Pressure intensity plots for the merging of two equal strength, like-signed vortices. $P_{n}=-6.0 \rho_{0}(\Gamma / \pi d)^{2}$. 


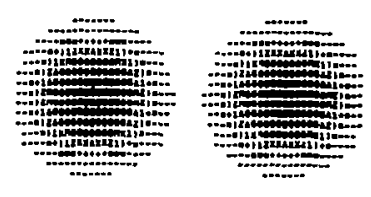

$$
\begin{aligned}
t \Gamma / \mathrm{d}^{2} & =0.0 \\
\zeta_{\max } / \zeta_{N} & =1.0
\end{aligned}
$$
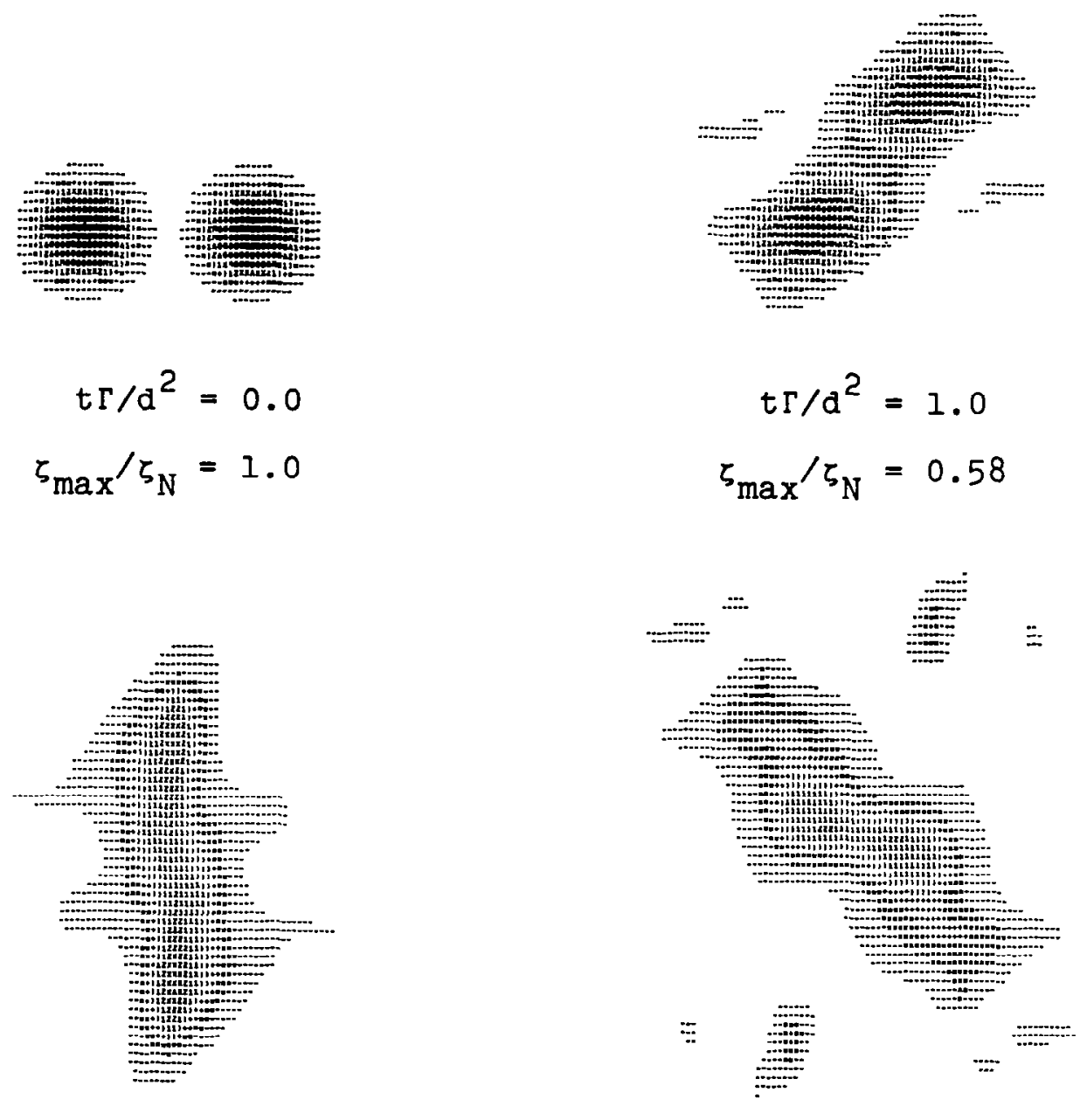

$$
\begin{aligned}
t \Gamma / d^{2} & =2.0 \\
\zeta_{\max } / \zeta_{N} & =0.43
\end{aligned}
$$

$$
\begin{aligned}
t \Gamma / d^{2} & =3.0 \\
\zeta_{\max } / \zeta_{N} & =0.35
\end{aligned}
$$

F1gure 32. The mean vorticity distribution during the merging of two like-sign, equal strength vortices. $2 \zeta_{N} r / \pi d^{2}=7 \cdot 39$ 

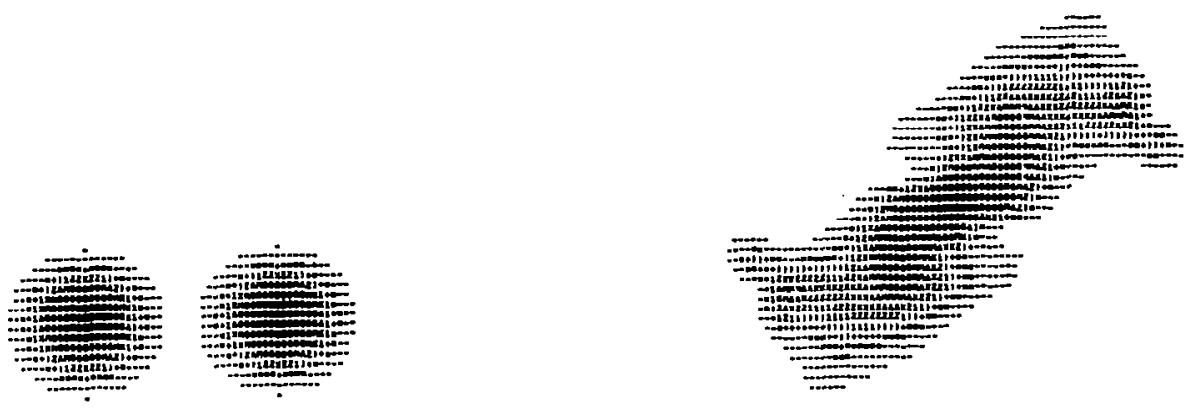

$$
\begin{aligned}
\mathrm{t} \Gamma / \mathrm{d}^{2} & =0.0 \\
\mathrm{q}_{\max }^{2} / \mathrm{q}_{N} & =1.0
\end{aligned}
$$

$$
\begin{aligned}
t \Gamma / d^{2} & =1.0 \\
q_{\max }^{2} / q_{N}^{2} & =1.15
\end{aligned}
$$
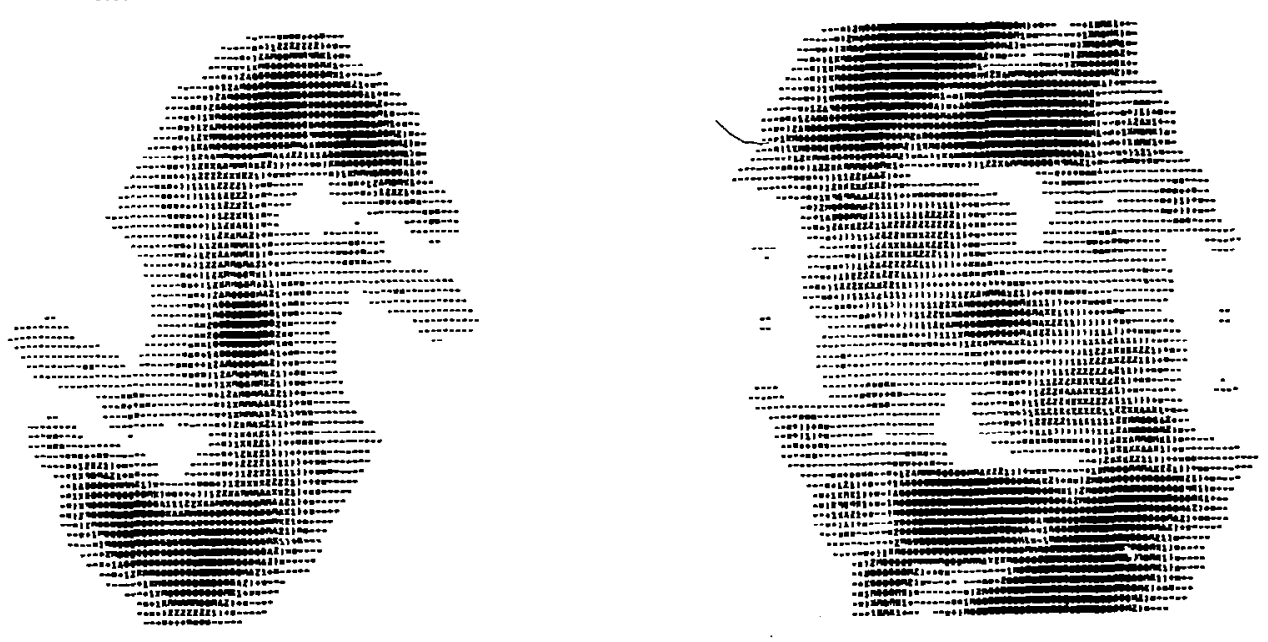

$$
\begin{aligned}
t \Gamma / d^{2} & =2.0 \\
q_{\max }^{2} / q_{N}^{2} & =1.63
\end{aligned}
$$

$$
\begin{aligned}
t \Gamma / d^{2} & =3.0 \\
q_{\max }^{2} / q_{N}^{2} & =2.8
\end{aligned}
$$

Figure 33. Twice the turbulent kinetic energy distribution during the merging of two like-sign and equalstrength vortices. $\left(q_{N} \pi d / \Gamma\right)^{2}=0.01$ 
A comparison with an equivalent isolated vortex decay computation illustrates the significance of the merging phenomenon in terms of aging the vortex flow field. We have chosen to compute the decay of an isolated axisymmetric vortex whose inftial vorticity distribution is Gaussian and whose circulation equals that of the pair. The core radius $r_{c}$ has been chosen to make the polar moment of the vorticity distribution computed about the centroid equal to that of the merging pair. Thus,

$$
r_{c}^{2}=r_{c_{\text {pair }}}^{2}+\frac{d^{2}}{4}
$$

The computation is carried out with an axisymmetric version of the code (ref. 28) used to compute the merging of the like-signed pair. The initial turbulence distribution is taken to be $\overline{u u}=\overline{v v}=\overline{w W}=$ $q^{2} / 3$ where

$$
q^{2}=2.0 q_{0}^{2} \exp \left[-\left(\frac{r}{r_{c p a i r}}\right)^{2}\right]
$$

so that the initial total turbulent kinetic energies in both computations are equal. The integral scale parameter $\Lambda$ is taken to be $0.1 d$.

Figure 34 shows the total turbulent kinetic energy in the crossplane as a function of time. The level that can be supported by the axisymmetric isolated vortex is far less than initially introduced, as discussed earlier and in reference 2. However, the breakdown of the axisymmetric structure about each vortex in the merging pair results in the production of turbulent kinetic energy and, hence, Reynolds stresses, which diffuse the mean vorticity outward in addition to the convective spreading. The production of turbulence is the transport mechanism which can diffuse vorticity to the wake centerline and result in the decay of circulation in the wake, as was shown schematically in figure 1 .

Calculations involving merging of unequal strength vortices have been undertaken with the second vortex $50 \%, 20 \%$, and $-20 \%$ the strength of the first vortex and with all other initial conditions taken to be the same as those used in the merging calculation of two like-sign, equal-strength vortices. The comparison between the merging of equal-sign and strength vortices and merging with the second vortex $50 \%$ of the strength of the first can be made by comparing figure 32 with 35 and figure 33 with 36 . As can be seen, merging of equal-strength vortices involves equal redistribution of both vorticity distributions, while the $50 \%$ vortex in the unequal merging computation is convected about the strong vortex. The structure of the strong vortex is not altered during this process. It is interesting to note that while the merging of the 


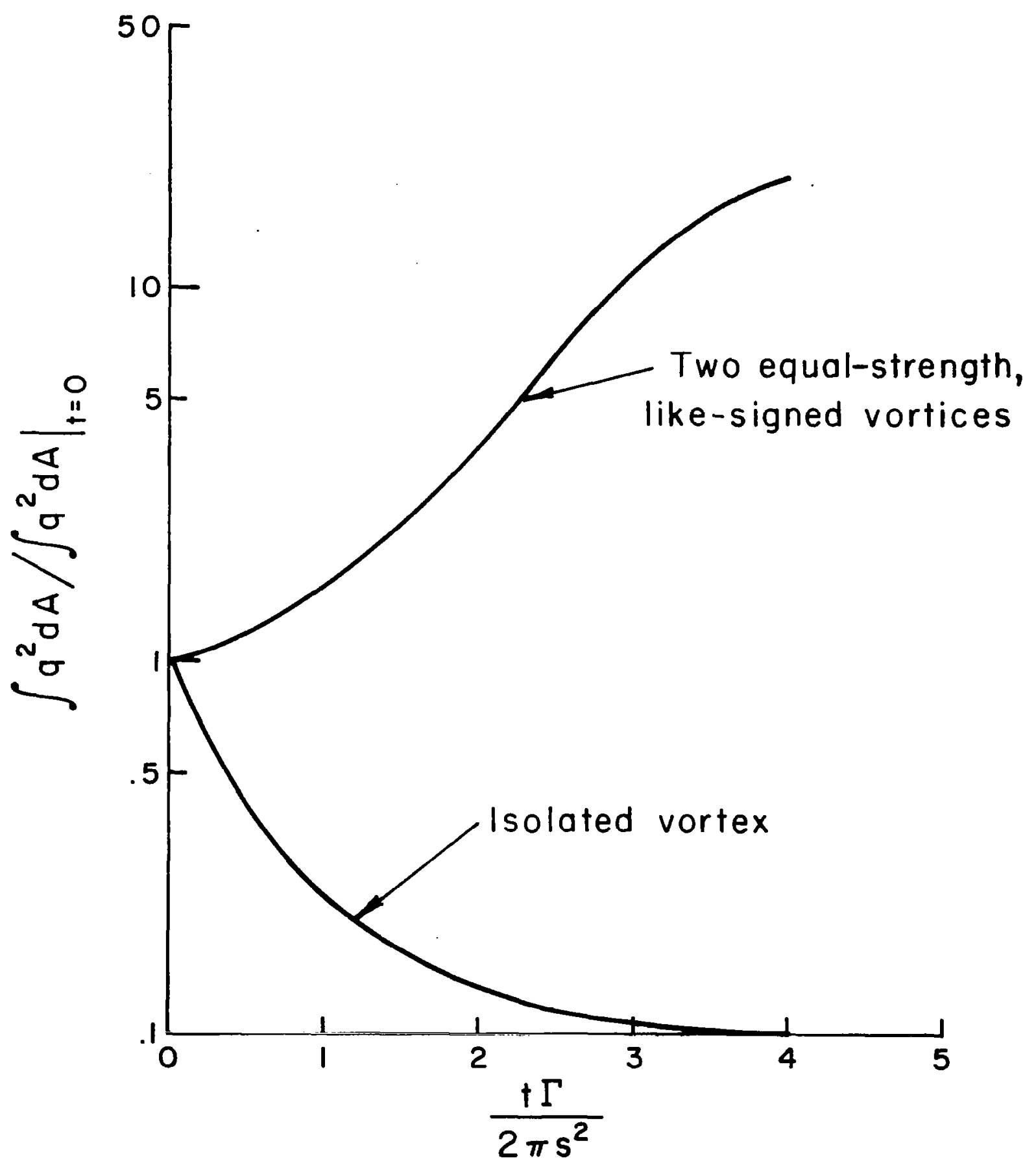

Figure 34. Comparison of the turbulent kinetic energy as a function of time between an isolated vortex and two equal-strength, like-sign vortices 


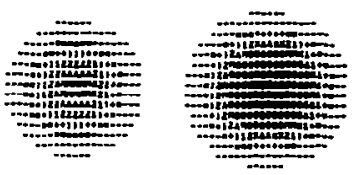

$$
\begin{aligned}
t \Gamma / d^{2} & =0.0 \\
\zeta_{\max } / \zeta_{N} & =0.98
\end{aligned}
$$

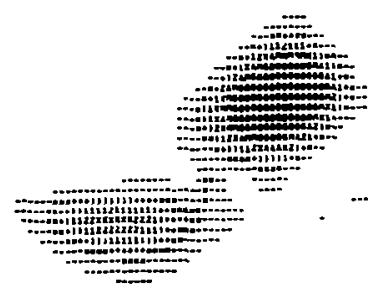

$$
\begin{aligned}
t \Gamma / d^{2} & =1.0 \\
\zeta_{\max } / \zeta_{N} & =0.75
\end{aligned}
$$
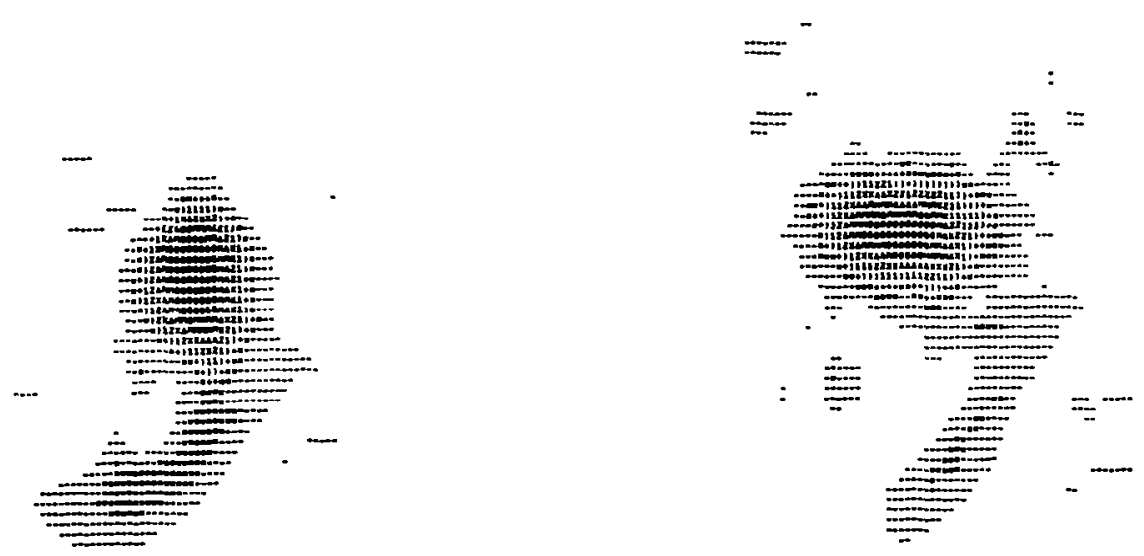

$$
\begin{aligned}
t \Gamma / d^{2} & =2.0 \\
\zeta_{\max } / \zeta_{N} & =0.605
\end{aligned}
$$

$$
\begin{aligned}
t \Gamma / d^{2} & =3.0 \\
\zeta_{\max } / \zeta_{N} & =0.59
\end{aligned}
$$

Figure 35. The mean vorticity distribution during the merging of two like-sign vortices. The vortex on the left is one-half the strength of the vortex on the right. $2 \zeta_{N} \Gamma / \pi d^{2}=7.39$ 

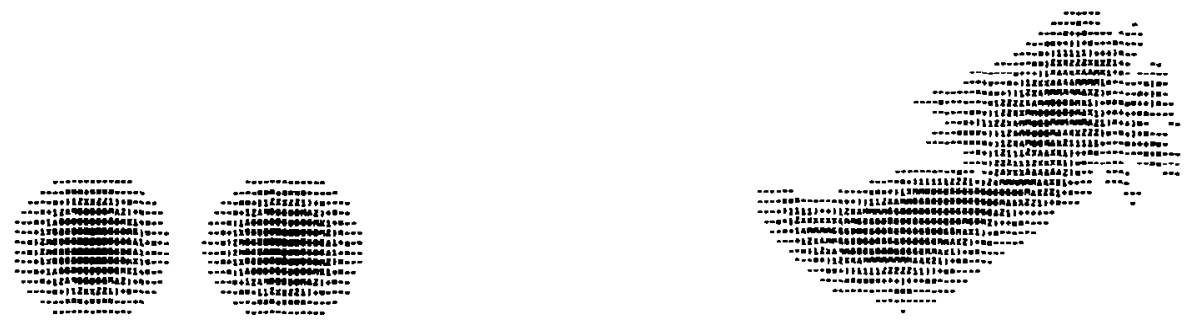

$$
\begin{aligned}
t \Gamma / \mathrm{d}^{2} & =0.0 \\
\mathrm{q}_{\max }^{2} / \mathrm{q}_{N}^{2} & =1.0
\end{aligned}
$$

$$
\begin{aligned}
t \Gamma / d^{2} & =1.0 \\
q_{\max }^{2} / q_{N}^{2} & =0.7
\end{aligned}
$$
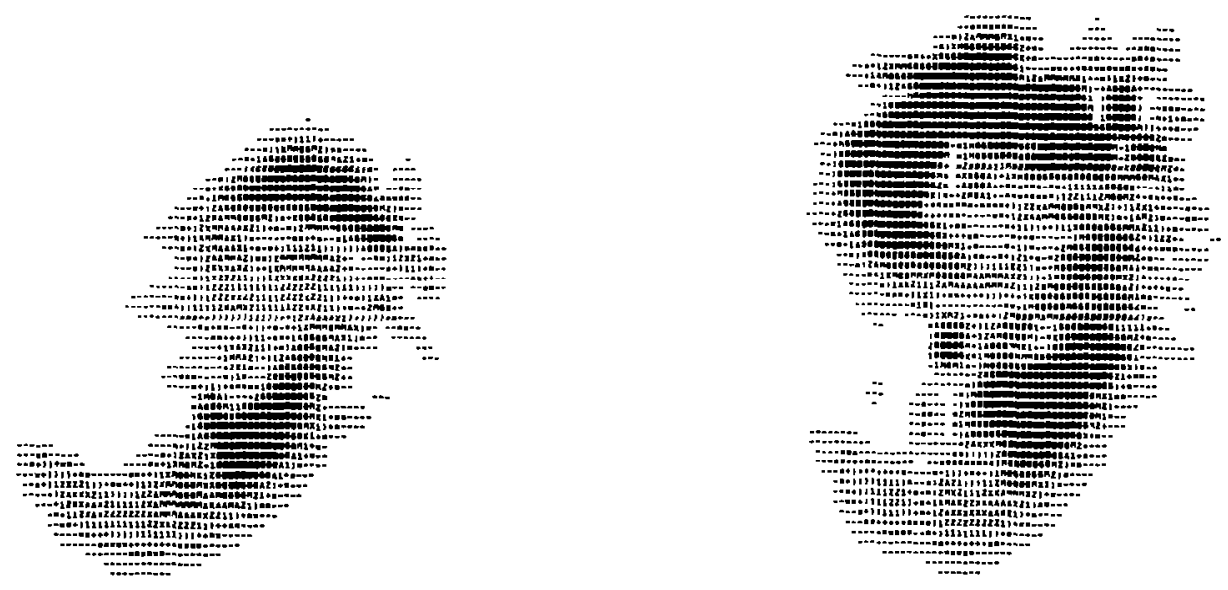

$$
\begin{array}{r}
t \Gamma / d^{2}=2.0 \\
q_{\max }^{2} / q_{N}^{2}=2.0
\end{array}
$$

$$
\begin{array}{r}
t \Gamma / d^{2}=3.0 \\
q_{\max }^{2} / q_{N}^{2}=4.9
\end{array}
$$

Figure 36. Twice the turbulent kinetic energy distribution during the merging of two like-sign vortices. The vortex on the left is one-half the strength of the vortex on the right. $\left(q_{N} \pi d / \Gamma\right)^{2}=0.01$ 
unequal strength vortices produces a higher local level of turbulent kinetic energy, the spread of the turbulent kinetic energy is greater in the like-sign, equal-strength computation. The results for the merging of the $20 \%$ and $-20 \%$ strength second vortices are not shown here, but we note that the merging redistribution is qualitatively the same as observed for the unequal computation. The weaker vortex is convected around the stronger vortex with very little change in the structure of the stronger vortex.

A minimum hazard vortex wake.- The results given above may be used to determine a minimum hazard aircraft wake. The only assumption which is made is to neglect the effect of the opposite merging pair since the first-order effect of the vortices from the opposite wing is to bring about wake descent. This, however, is not true if a vortex is sufficiently close to the aircraft centerline so that its mate dominates. This would be the case in which two vortex pairs would not remain together; it has been discussed in Section 2.4 with the aid of figure 8 . However, here we are interested in merging and are looking for a configuration where the two vortex pairs remain together.

If lift is held constant in evaluating the hazard between various merging wakes, then

$$
L=\rho U_{\infty} 2 \bar{y}\left(\Gamma_{I}+\Gamma_{2}\right)
$$

with the positions of the vortices shown below.

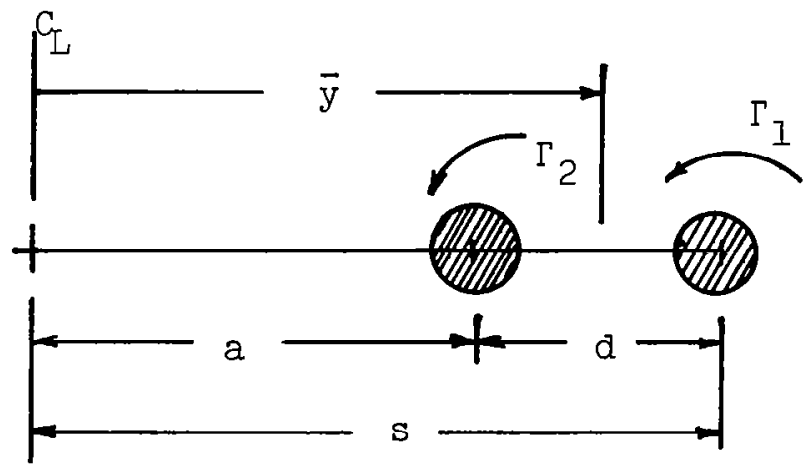

The centroid of the system is computed to be

$$
\bar{y}=\frac{a \Gamma_{2}+s \Gamma_{1}}{\Gamma_{1}+\Gamma_{2}}
$$


Substituting into (33) yields

$$
\frac{L}{2 \rho U_{\infty}}=\Gamma_{I} S\left[\left(I-\frac{d}{s}\right) f+I\right]
$$

where $f=\Gamma_{2} / \Gamma_{1}$.

Vortex hazard is evaluated by NASA by measuring the roling moment induced on a following wing by the vortex wake. Although the calculation of aerodynamic loads on a wing using strip theory is of limited value even in uniform flow, it is adequate if trends and minimums are to be obtained. The torque induced on a constant chord airfoil is

$$
T=\int_{-s_{f}}^{s_{f}} c_{\ell_{\alpha}} \frac{W}{U_{\infty}} \cdot q c_{f} y d y
$$

Expressed as a rolling moment coefficient and with substitution of eq. (35),

with

$$
\begin{aligned}
C_{\ell} & =\frac{c_{\ell}{ }_{\alpha} C_{L}}{16 \pi} \frac{b^{2} d I}{b_{f}^{2} s[(I-d / s)+I]} \\
I & =\frac{4 \pi}{\Gamma_{I} d} \int_{-2 s_{f} / d}^{2 s_{f} / d} W y d y
\end{aligned}
$$

I is the nondimensional ro r.ing moment computed in the Vortex Wake code and the subscript $f$ denotes follower aircraft. The decay of the maximum rolling moment encountered as a function of downstream distance for the case where the wing span of the follower aircraft is $20 \%$ of the wing span of the generator is shown in figures 37,38 , and 39. The first observation which is apparent from each of these figures is that the rolling moment is a minimum when the merging vortices are of equal strength. Selection of the spacing $d / s$ is not as easy, with $d / s=0.6$ and 0.8 comparing quite closely. However, the $d / s=0.6$ spacing shows a slight advantage. If we refer to figure 12 , the $\mathrm{d} / \mathrm{s}$ beyond which equalstrength vortex pairs will not remain together is about 0.8 , so that the equal strength with separation $d / s=0.6$ will be a minimum hazard wake for a follower aircraft which has a span that is $20 \%$ of the span of the generating aircraft.

The oscillations which occur in the rolling moment as a function of downstream distance are expected and are associated with 


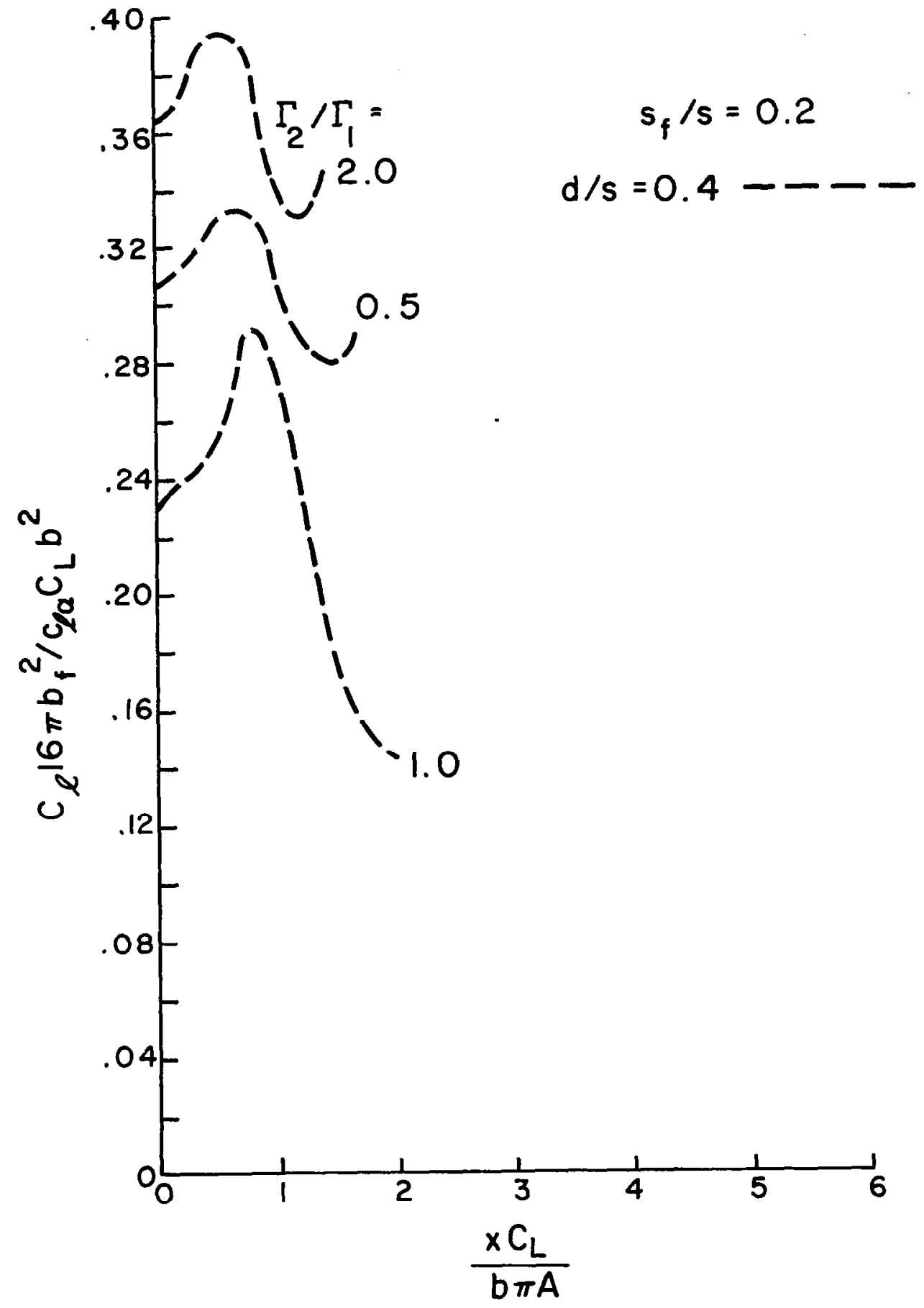

F1gure 37. Decay of the maximum rolling moment encountered by a following aircraft of semispan $20 \%$ of the semispan of the generating alrcraft $(d / s=0.4)$ 


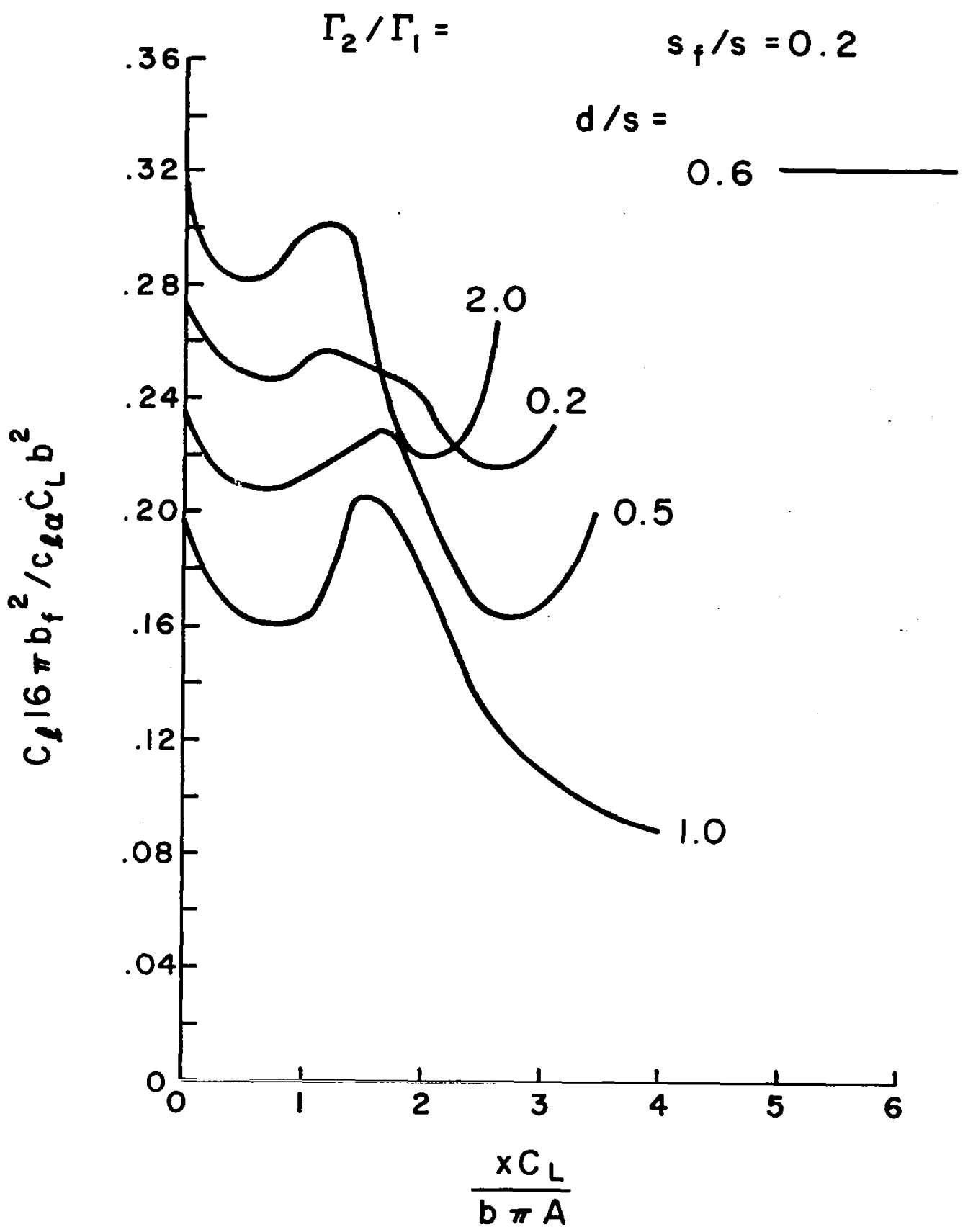

Figure 38. Decay of the maximum rolling moment encountered by a following alrcraft of semispan $20 \%$ of the semispan of the generating aircraft $(d / s=0.6)$ 


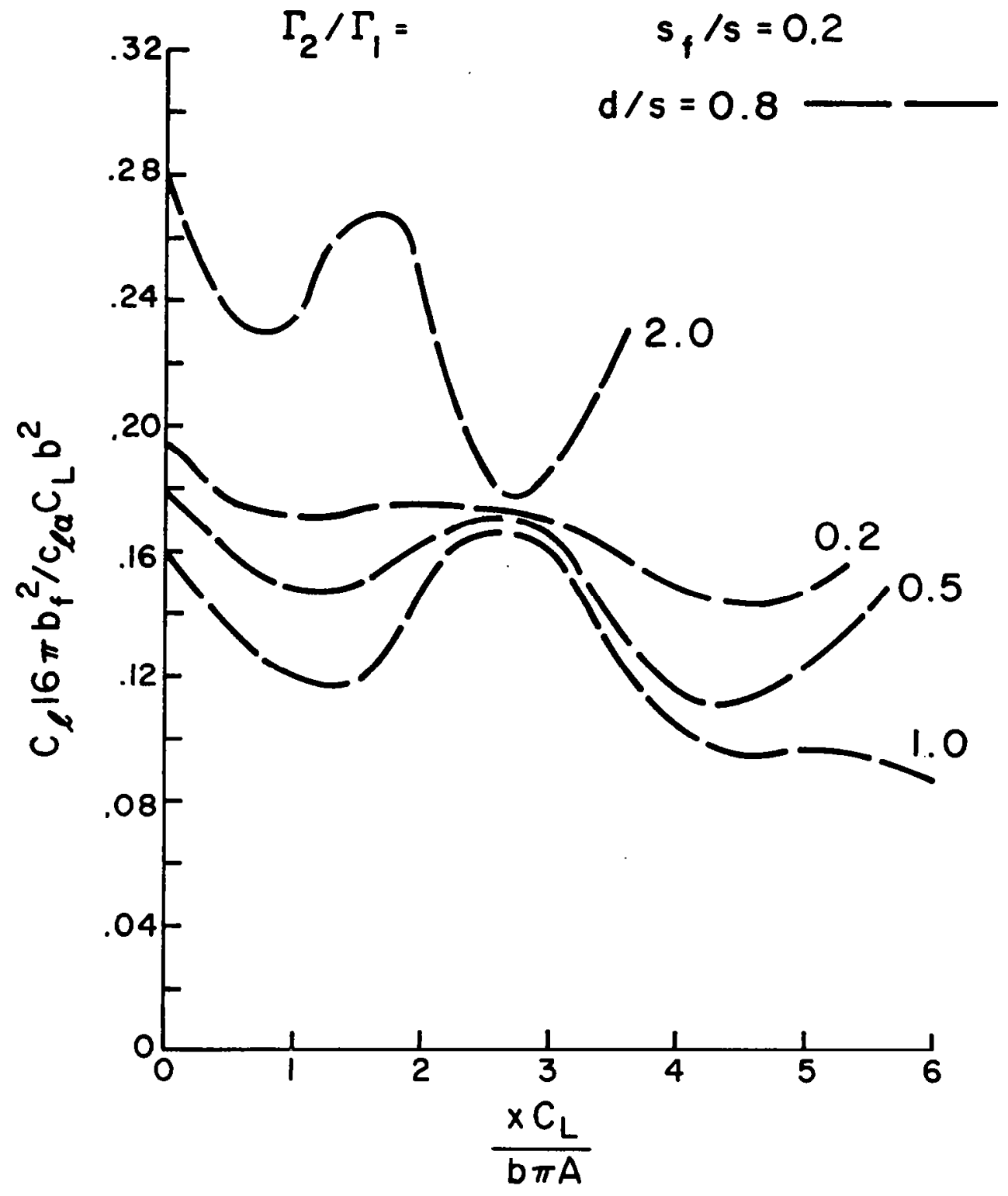

F1gure 39. Decay of the maximum rolling moment encountered by a following alrcraft of semispan $20 \%$ of the semispan of the generating alrcraft $(d / s=0.8)$ 
the orientation of the unmerged distributions of vorticity relative to the following aircraft. As the merging process goes to completion, the amplitude of the oscillation will decay, with the average value of the rolling moment (averaged between peaks and troughs) decaying smoothly. This can begin to be seen in figure 39 for the equal-strength roliing moment curve.

If the following aircraft has a span which is $60 \%$ of the span of the generator, figures 40 and 41 show the decay of the rolling moment with downstream distance. Again, the equal-strength merging results in a minimum induced rolling moment at a given $d / s$. However, as is expected, the difference in level between various relative vortex strengths is not as significant as it is for the smaller following aircraft. The results for d/s are not shown here since they induce very high levels of rolling moment. There is little difference in rolling moment levels between the $d / s=$ 0.6 and 0.8 vortex separations.

The results of the calculations given above indicate that the minimum hazard wake for a small follower aircraft is achieved by having equal-strength flap and tip vortices, with the tip vortices located at $y= \pm s$ and the flap vortices located at $y= \pm 0.4 \mathrm{~s}$. This finding is a major result of the effort reported here. Experimental verification of it should guide future experimental efforts.

A line vortex immersed in a simple shear flow.- Moore and Saffman (ref. 8) have found the solution to a uniform vorticity vortex in a simple shear flow. They have shown that, provided the simple shear is not too large compared to the vorticity in the vortex, the vortex takes an elliptic cross-section. The interesting result is that when the vorticity associated with the simple shear has the same sense as the vorticity in the vortex the major axis of the ellipse is parallel to the streamlines at infinity. When the vorticity associated with the shear is of sense opposite to the vorticity in the vortex, the major axis of the ellipse is normal to the streamlines at infinity.

This result was checked qualitatively, using the Vortex Wake Code, by placing a vortex at $y=z=0$ with a Gaussian distribution of vorticity and superimposing a horizontal velocity equal to $\mathrm{Bz}$. The initial turbulence distribution was taken from the superequilibrlum limit (ref. 35) of the second-order closure model. The turbulent energy components are

$$
\overline{v v}=2 \overline{u u}=2 \overline{w w}=\Lambda^{2}\left|\frac{d V}{d z}\right|^{2}
$$

with the cross-correlation equal to $\overline{v w}=-0.35 \Lambda^{2}|\mathrm{dV} / \mathrm{dz}|^{2}$. The only mean flow length scale in the problem is $r_{c}$, and the integral scale parameter $\Lambda$ was taken to be $\Lambda=0.4 r_{c}$. 


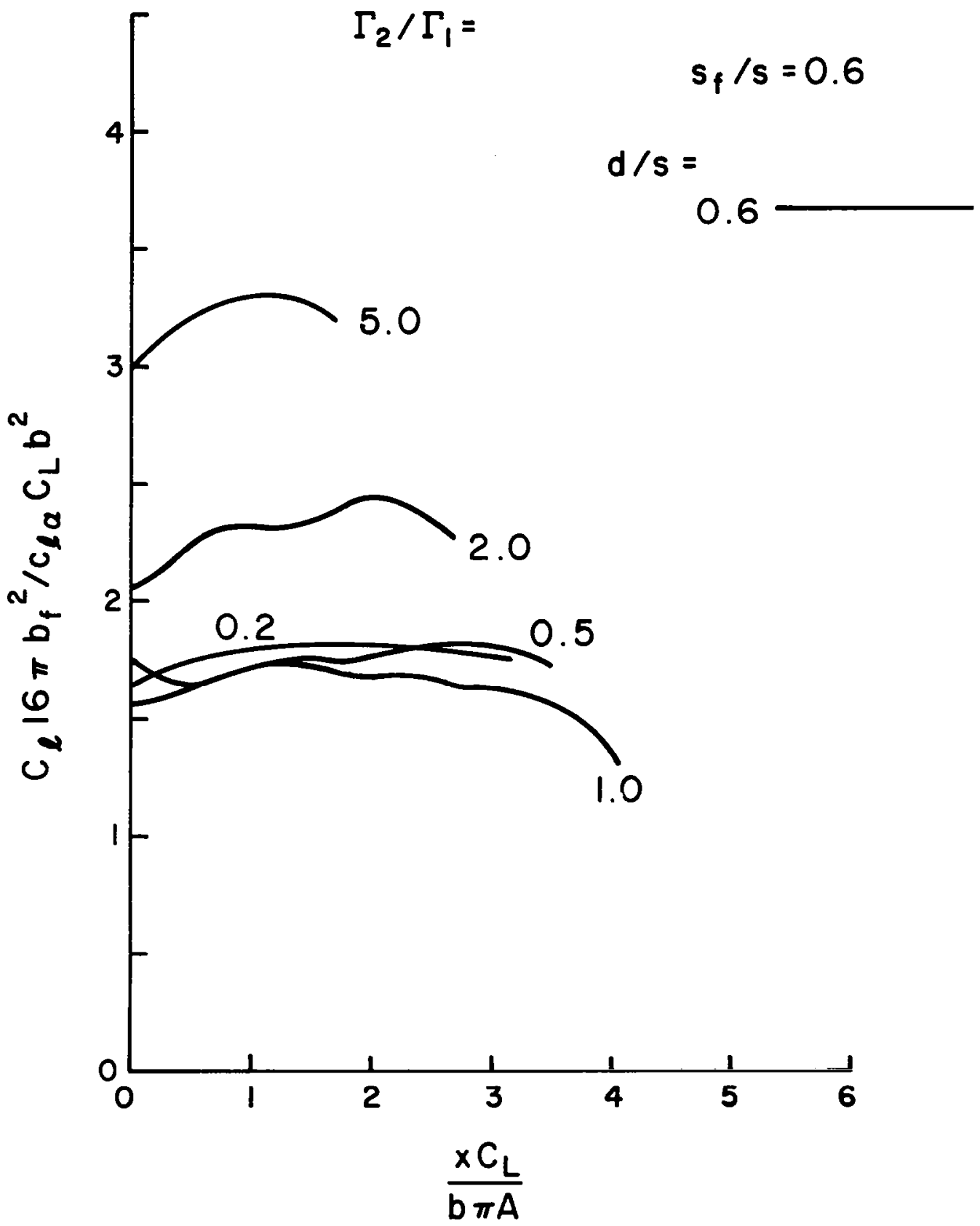

Figure 40. Decay of the maximum rolling moment encountered by a following alrcraft of semispan $60 \%$ of the semispan of the generating alrcraft $(d / s=0.6)$ 


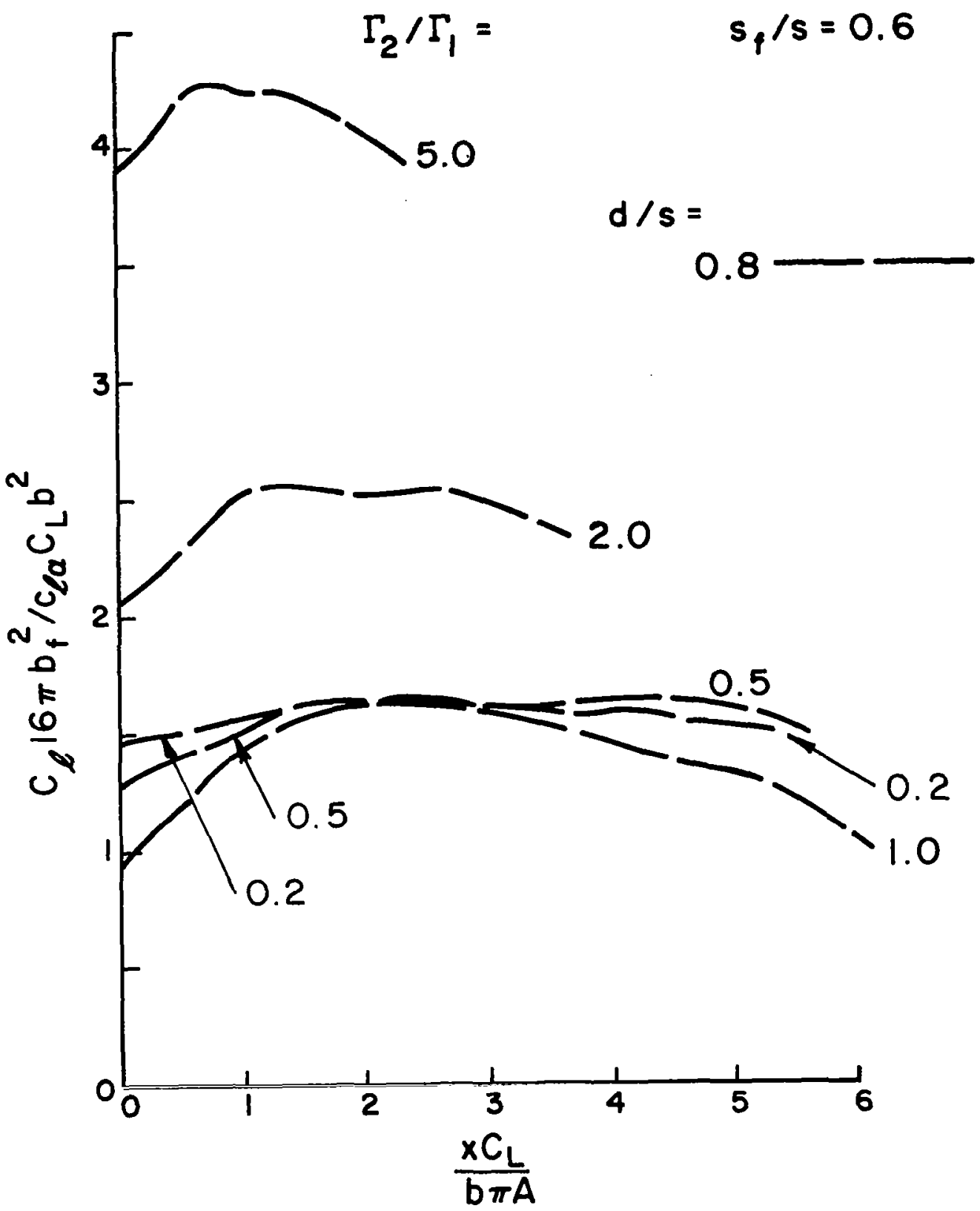

Figure 41. Decay of the maximum rolling moment encountered by a following alrcraft of semispan $60 \%$ of the semispan of the generating aircraft $(d / s=0.8)$ 
Results will be given for two cases $8 \pi \beta r_{c}^{2} / \Gamma= \pm 0.5$. In figure 42 a comparison is shown between the vorticity of a vortex immersed in a positive simple shear flow $\beta<0$ and a negative simple shear flow $\beta>0$ (the vorticity in the shear flow is subtracted so that the level shown is that of the initial Gaussian of vorticity). As can be seen, the results obtained by Moore and Saffman (ref. 8) are qualitatively substantiated. No quantitative comparison can be made, however, since their analysis appears to be tractable only when the vorticity in the vortex is uniform; numerically a uniform vorticity vortex is difficult to discretize.

A comparison of the turbulence levels is shown in figure 43 . At the end of the computation, it can be seen that the level of turbulence is greater in the vortex which is immersed in a shear which is in opposite sense to the vortex. This might have been suspected since the positive vortex is essentially immersed in a negative vorticity bath--a situation where axisymmetry is well known to be statically unstable. However, the issue of the production of turbulence by a vortex in a simple shear is more complicated due to the fact that sufficient shear will convect the vortex apart; the physical situation is far from being axisymmetric.

Merging in aircraft wakes.- The results of three calculations simulating the vortex interactions in the wake of aircraft are presented here. The first computation follows the merging interaction of a flap and tip vortex of like sign and equal strength. The tip vortices are positioned at $y= \pm 0.95 \mathrm{~s}$ and the flap vortices at $y= \pm 0.4 \mathrm{~s}$. The vortices were given Gaussian distributions of vorticity with strength $\Gamma$, and $r_{c}=0.2$ s for all vortices. Gaussians of turbulent kinetic energy were centered in each vortex with

$$
\overline{u u}=\overline{v v}=\overline{w w}=0.033\left(\frac{\Gamma}{2 \pi s}\right)^{2} \exp \left(-r^{2} / r_{c}^{2}\right)
$$

and cross-correlations were initially taken to be zero. The integral scale parameter was initially taken to be $\Lambda / s=0.2$ and $\Gamma / \nu=10^{4}$. Results for the distribution of vorticity and turbulent kinetic energy are shown in figures 44 and 45 , respectively.

These distributions may be compared with the results of a second computation where the strength of the tip vortex is reduced to $30 \%$ of the strength of the flap vortex. All other initial conditions remain the same. This case is shown in figures 46 and 47 .

As a third computation, a negative fuselage vortex of $47 \%$ the strength of the flap vortex, positioned at $y / s= \pm 0.2$, is added to the initial conditions of the second computation. This vortex simulates the vortex trailed as a result of the lift dropoff at the 


$$
\begin{gathered}
8 \pi B r_{c}^{2} / \Gamma=0.5 \\
\zeta / \zeta_{n}=1.0
\end{gathered}
$$

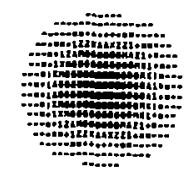$$
8 \pi B r_{c}^{2} / \Gamma=-0.5
$$

$$
t \Gamma / 2 \pi r_{c}^{2}=0.0
$$
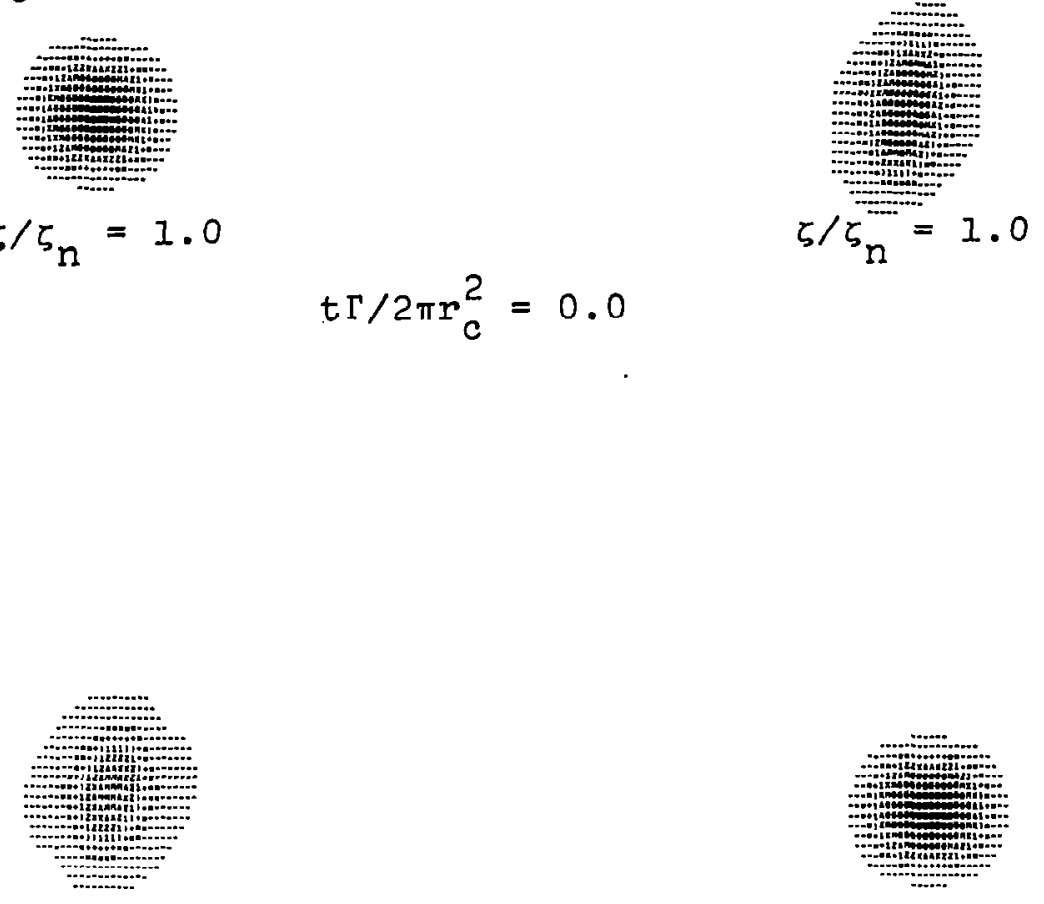

$$
\zeta / \zeta_{n}=0.76
$$

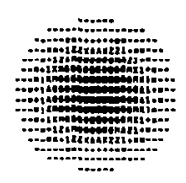

$$
\zeta / \zeta_{n}=0.72
$$

$$
t \Gamma / 2 \pi r_{c}^{2}=8.0
$$
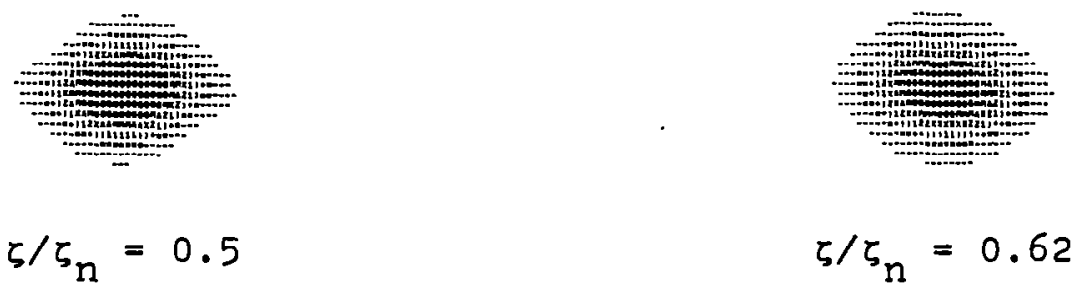

$t \Gamma / 2 \pi r_{c}^{2}=16.0$

Figure 42. Comparison of the decay of a Gaussian of vorticity immersed in a simple shear flow having the same sense as the vortex with a vortex immersed in a single shear flow having the opposite sense as the vortex $\left(\xi_{n} \Gamma / \pi r_{c}=32.0\right)$ 


$$
8 \pi r_{c}^{2} / \Gamma=0.5
$$

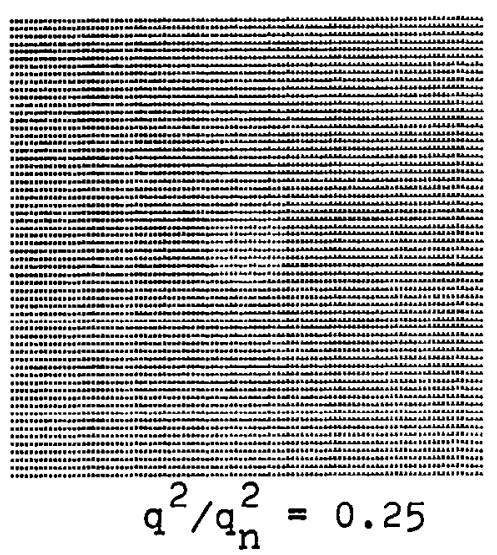

$$
8 \pi r_{c}^{2} / \Gamma=-0.5
$$

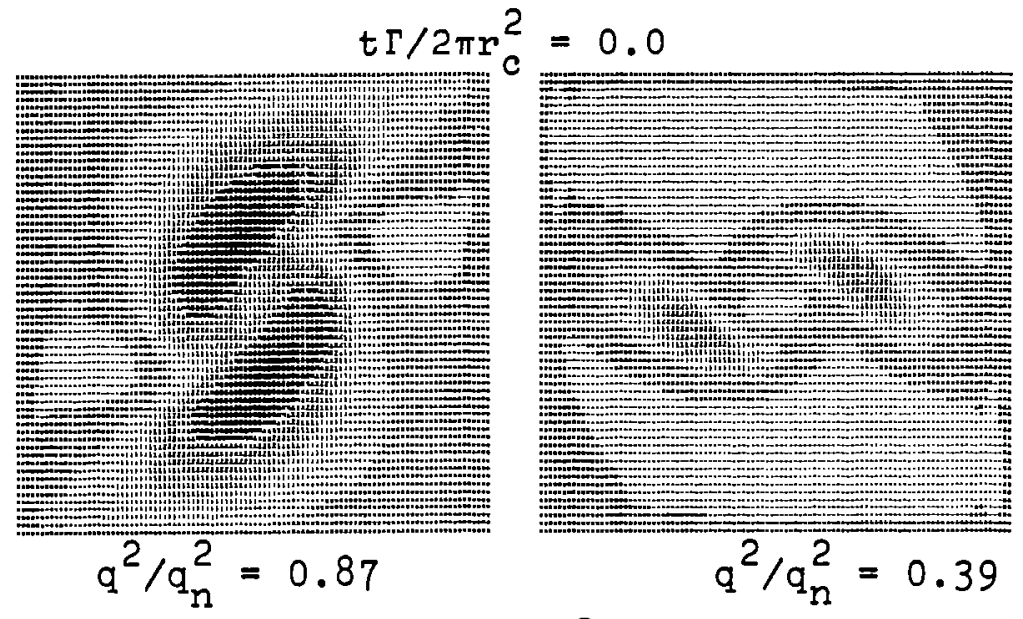

$$
t \Gamma / 2 \pi r^{2}=8.0
$$
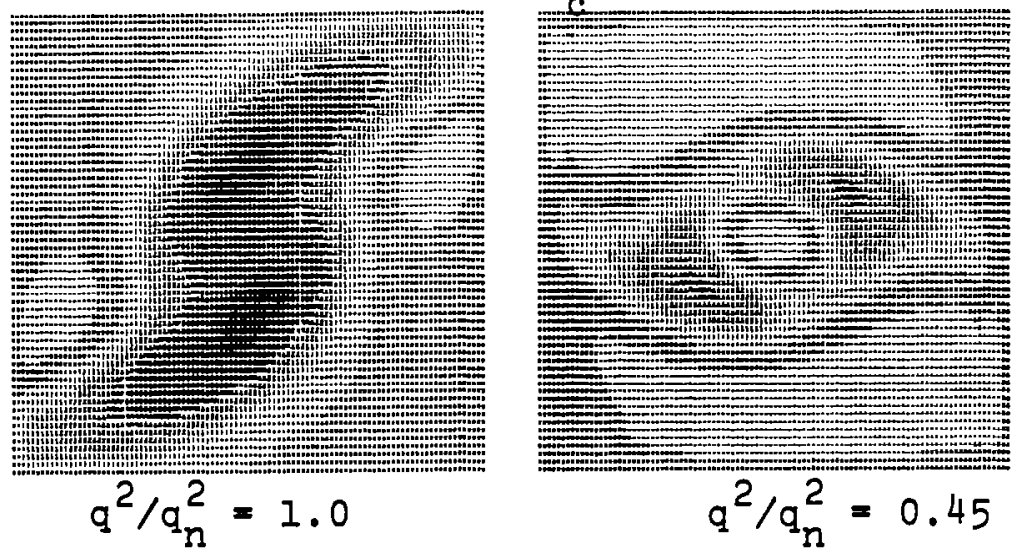

$$
q^{2} / q_{n}^{2}=1.0
$$

$$
q^{2} / q_{n}^{2}=0.45
$$

$$
t \Gamma / 2 \pi r_{c}^{2}=16.0
$$

F1gure 43. Comparison of the turbulent kinetic energy between a vortex Immersed in a simple shear of the same sense of the vortex with a vortex Immersed in a simple shear of the opposite sense of the vortex $\left(\left[q_{n} 2 \pi r_{c} / \Gamma\right]^{2}=0.09\right)$ 

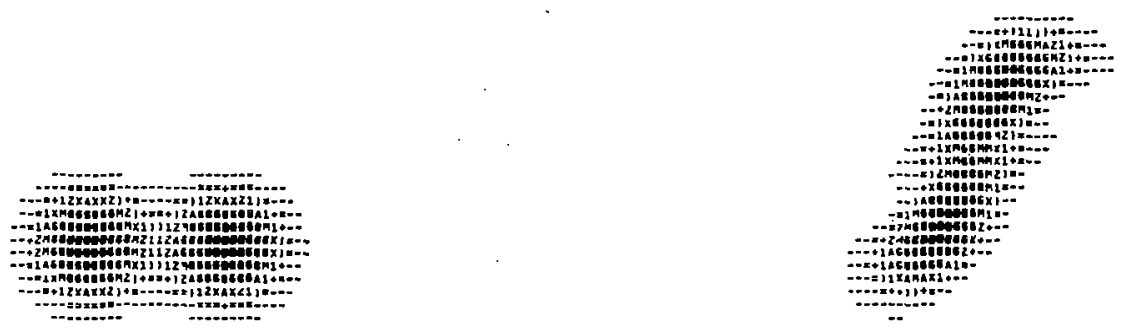

$$
\begin{aligned}
t \Gamma_{\mathrm{f}} / 2 \pi \mathrm{s}^{2} & =0 \\
\zeta_{\max } / \zeta_{\mathrm{n}} & =1.0
\end{aligned}
$$

$$
\begin{aligned}
t \Gamma_{\mathrm{f}} / 2 \pi \mathrm{s}^{2} & =0.2 \\
\zeta_{\max } / \zeta_{\mathrm{n}} & =0.92
\end{aligned}
$$
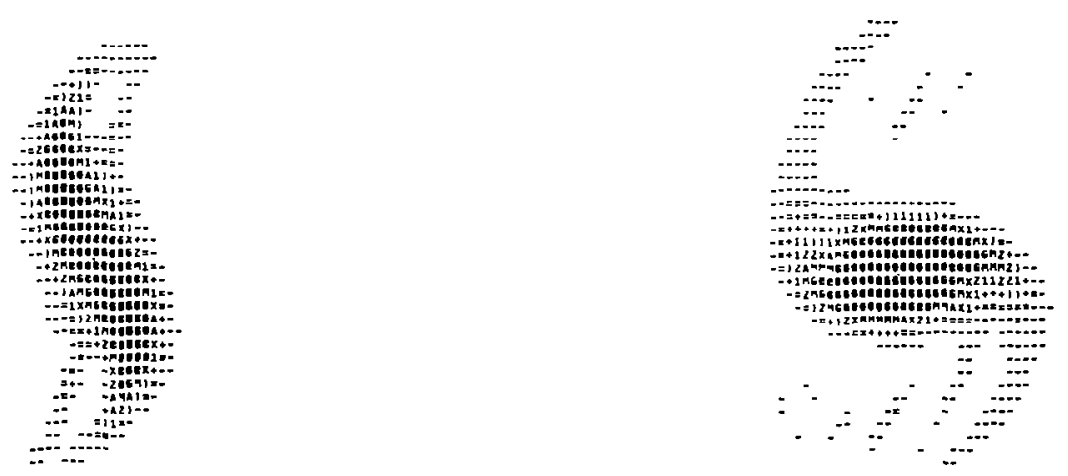

$$
\begin{aligned}
t \Gamma_{f^{\prime}} / 2 \pi s^{2} & =0.4 \\
\zeta_{\max } / \zeta_{n} & =0.87
\end{aligned}
$$$$
t \Gamma_{f^{\prime}} / 2 \pi s^{2}=0.6
$$$$
\zeta_{\max } / \zeta_{n}=0.82
$$

Figure 44. Distribution of trailed vorticity in the halfplane; flap and tip vortices are of the same sign and equal strength $\left(\zeta_{n}=50.0 \Gamma_{f} / 2 \pi s^{2}\right)$ 

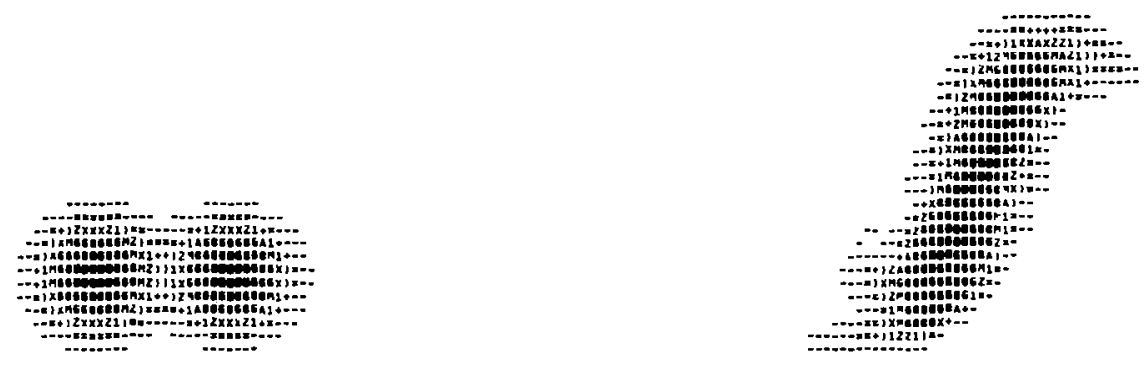

$$
\begin{aligned}
t \Gamma_{f} / 2 \pi s^{2} & =0 \\
q^{2} / q_{n}^{2} & =1.0
\end{aligned}
$$

$$
\begin{aligned}
t \Gamma_{f} / 2 \pi s^{2} & =0.2 \\
q^{2} / q_{n}^{2} & =1.1
\end{aligned}
$$
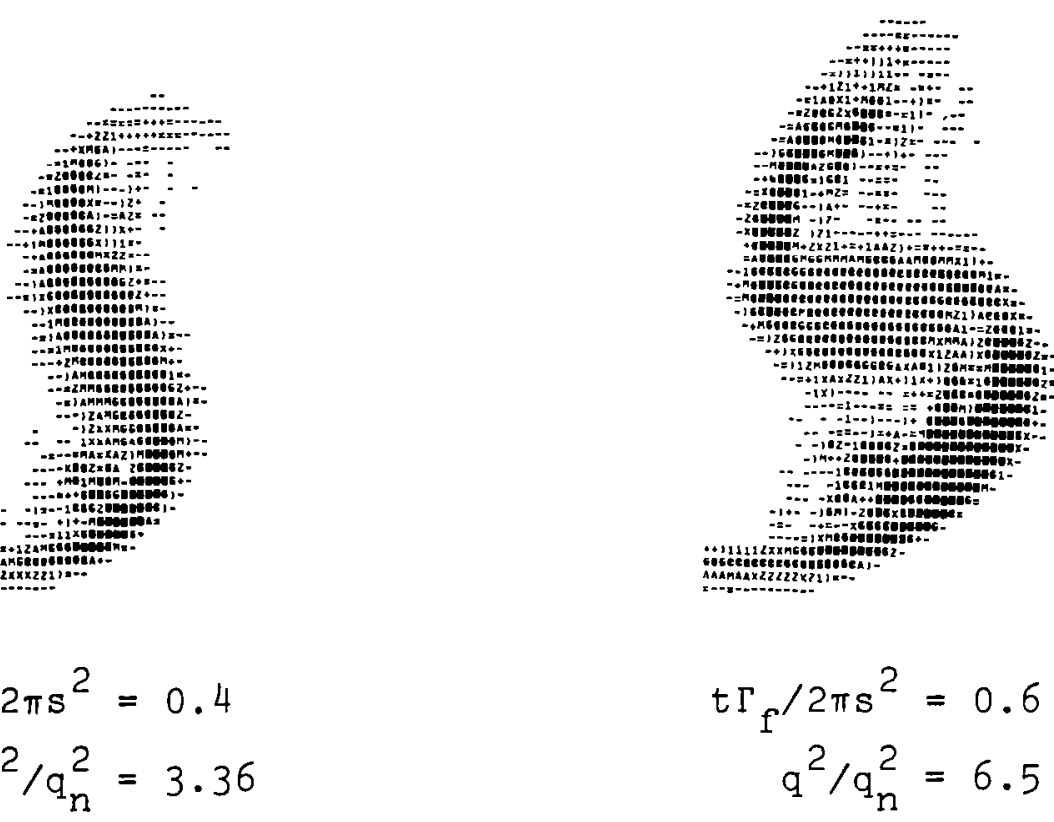

$$
\begin{aligned}
t \Gamma_{f} / 2 \pi s^{2} & =0.4 \\
q^{2} / q_{n}^{2} & =3.36
\end{aligned}
$$$$
q^{2} / q_{n}^{2}=6.5
$$

Figure 45. Production of turbulent kinetic energy during the merging of the vorticity distribution shown in figure $44\left[q_{n}^{2}=0.01\left(\Gamma_{f} / 2 \pi s\right)^{2}\right]$ 

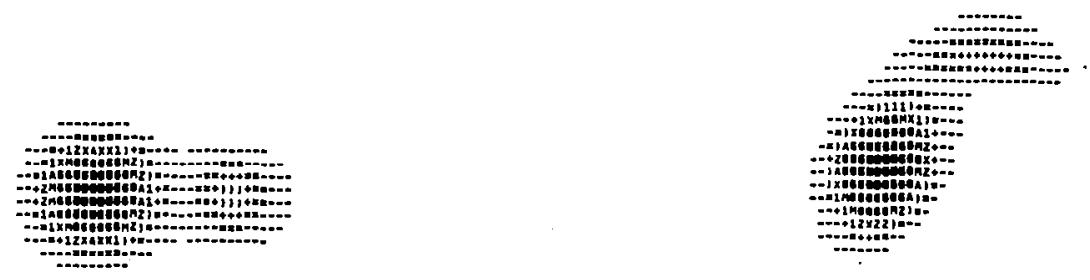

$t \Gamma_{f} / 2 \pi s^{2}=0$

$t \Gamma_{f} / 2 \pi s^{2}=0.2$

$$
\zeta_{\max } / \zeta_{n}=1.0
$$

$$
\zeta_{\max } / \zeta_{n}=0.9
$$
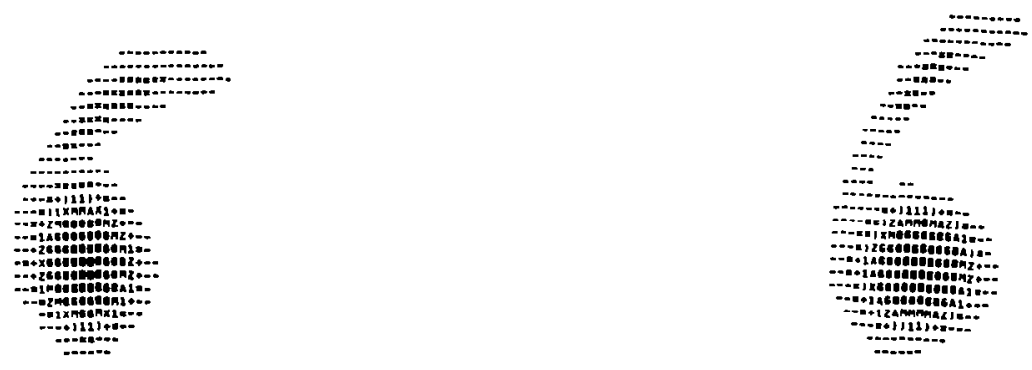

$$
\begin{aligned}
t \Gamma_{f^{\prime}} / 2 \pi s^{2} & =0.4 \\
\zeta_{\max } / \zeta_{n} & =0.86
\end{aligned}
$$

$$
\begin{aligned}
t \Gamma_{\mathrm{f}^{\prime}} / 2 \pi \mathrm{s}^{2} & =0.6 \\
\zeta_{\max } / \zeta_{\mathrm{n}} & =0.82
\end{aligned}
$$

Figure 46. Distribution of trailed vorticity in a half-plane; the tip vortex is $30 \%$ the strength of the flap vortex and both vortices are of the same sign $\left(\zeta_{n}=50.0 \Gamma_{f} / 2 \pi s^{2}\right)$ 

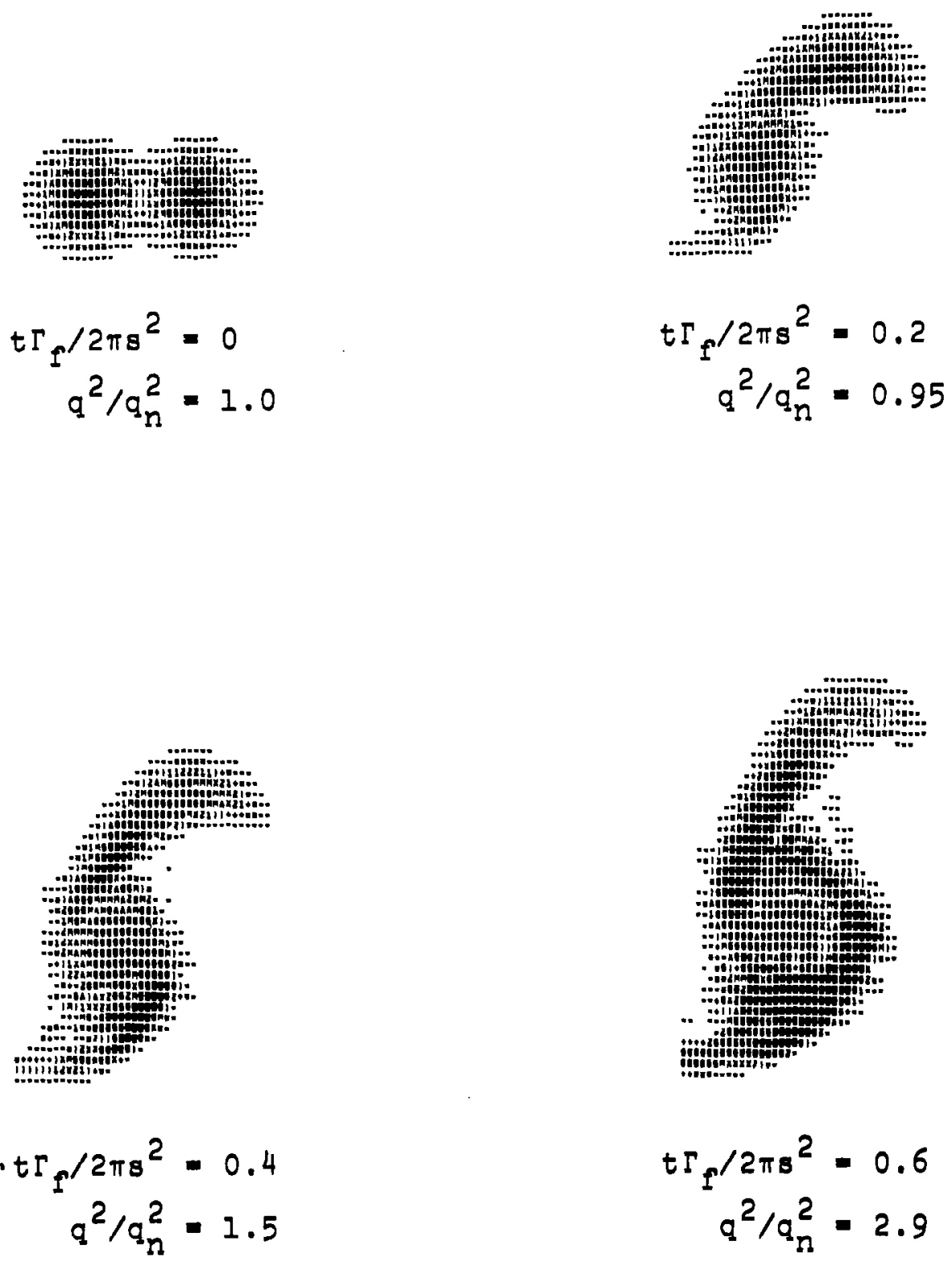

F1gure 47. Production of turbulent k1net1c energy during the merging of the vorticity distribution shown in f1gure $46\left[q_{n}^{2}=0.01\left(\Gamma_{f} / 2 \pi s\right)^{2}\right]$ 
wing-fuselage junction. The mean vorticity and turbulent kinetic energy distributions for this case are shown in figures 48 and 49 , respectively.

To make some quantitative comparison between these three cases in terms of deintensification, the results must be scaled so that lift is held constant between runs. To do this, the normalized vorticity in figures 46 and 48 must be scaled up by 2.0 and 2.3, respectively, while the nondimensional times in these figures must be scaled down by 2.0 and 2.3 , respectively. The net result is that the maximum level is about one-half as great for the case when equal-strength vortices are merged. More significant, however, is how extensively the trailed vorticity is spread by the merging phenomenon. The amount of shaded area in figures 44,46 , and 48 is an indicator of this, with the equal-strength merging case being most dispersed.

We note that in figure 49 the large level of turbulent kinetic energy occurs in a very small region inside the fuselage vortex which is being strained by the flap vortex; it does not contribute significantly to the total turbulent kinetic energy in the crossplane.

Engine exhaust.- It has been observed in full-scale flight tests that engine thrust levels do make a measurable change in the rate of decay of a vortex wake (ref. 36). It is reasonable to suspect that the basic merging phenomenon is not altered by the presence of jet exhausts, but that the additional turbulence produced by the exhaust jets results in additional turbulent diffusion of the trailed vorticity. To check this conjecture, cold engine exhausts were added to the equal-strength like-sign flap and tip vortex merging computation described above.

At a lift coefficient of 1.5 and assuming $C_{D}=C_{T}=0.125 C_{L}$, the axial velocity excess about each engine is taken to be

$$
\frac{\Delta U \pi s}{\Gamma f}=8.0 \exp \left(-52.0 \frac{r^{2}}{s^{2}}\right)
$$

Taking the aspect ratio equal to 7 , the flight speed is $40.0 \Gamma / 2 \pi \mathrm{s}$. The engine exhaust Gaussians of excess axial velocity are positioned at $\mathrm{y} / \mathrm{s}=-0.75,-0.4,0.4$, and 0.75 and $\mathrm{z} / \mathrm{s}=-0.08$. Except for a vertical displacement of $\mathrm{z} / \mathrm{s}=-0.08$, the inboard engines exhaust directly into the flap vortices. Additional Gaussian distributions of turbulent kinetic energy are positioned at each engine with the distribution given by

$$
\left(\frac{2 \pi \mathrm{sq}}{\Gamma_{f}}\right)^{2}=0.64 \exp \left(-52.0 \frac{\mathrm{r}^{2}}{\mathrm{~s}^{2}}\right)
$$



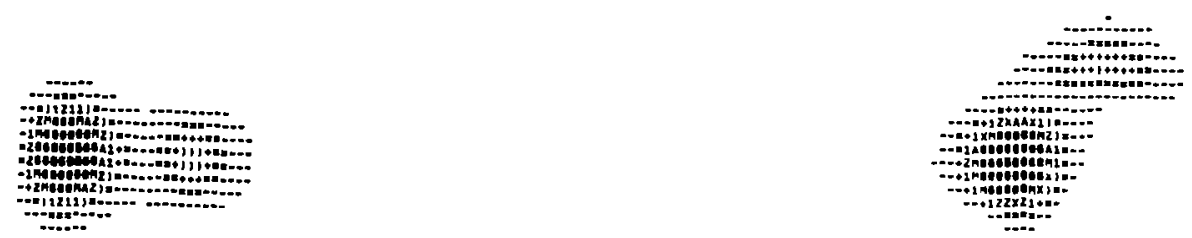

$$
\begin{aligned}
t \Gamma_{f^{\prime}} / 2 \pi s^{2} & =0.0 \\
\zeta_{\max } / \zeta_{n} & =0.84
\end{aligned}
$$$$
t \Gamma_{f} / 2 \pi s^{2}=0.2
$$

$$
\zeta_{\max } / \zeta_{n}=0.77
$$
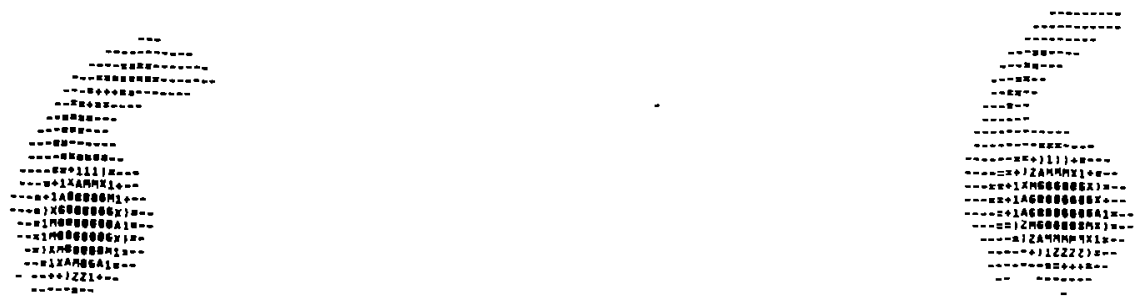

$$
\begin{aligned}
t \Gamma_{f} / 2 \pi s^{2} & =0.4 \\
\zeta_{\max } / \zeta_{n} & =0.74
\end{aligned}
$$

$$
\begin{aligned}
t \Gamma_{f^{\prime}} / 2 \pi s^{2} & =0.6 \\
\zeta_{\max } / \zeta_{n} & =0.69
\end{aligned}
$$

Figure 48. Distribution of trailed vorticity in a half-plane. The tip vortex is $30 \%$ the strength of the flap vortex and of the same sign. A negative fuselage vortex is $47 \%$ the strength of the fuselage vortex inftially located at $\mathrm{y} / \mathrm{s}=0.2$ (negative vortex is not shown since black regions denote positive vortic1ty $). \quad\left(\zeta_{\mathrm{n}}=50.0 \Gamma_{\mathrm{f}} / 2 \pi \mathrm{s}^{2}\right)$ 

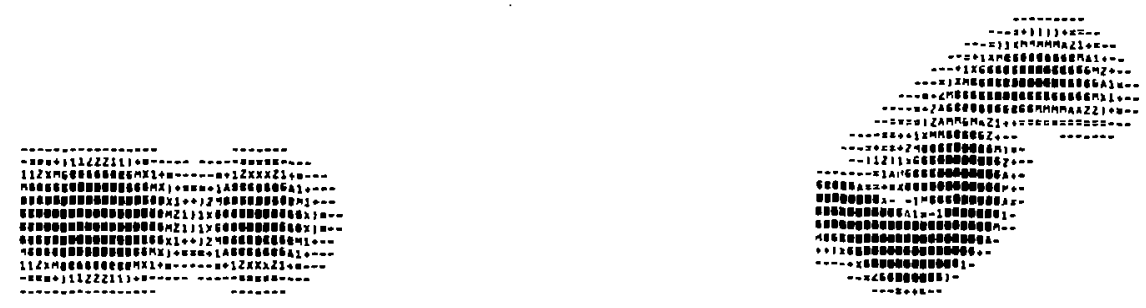

$$
\begin{aligned}
t \Gamma_{f} / 2 \pi s^{2} & =0.0 \\
q^{2} / q_{n}^{2} & =1.56
\end{aligned}
$$

$\begin{aligned} t \Gamma_{f} / 2 \pi s^{2} & =0.2 \\ q^{2} / q_{n}^{2} & =5.0\end{aligned}$
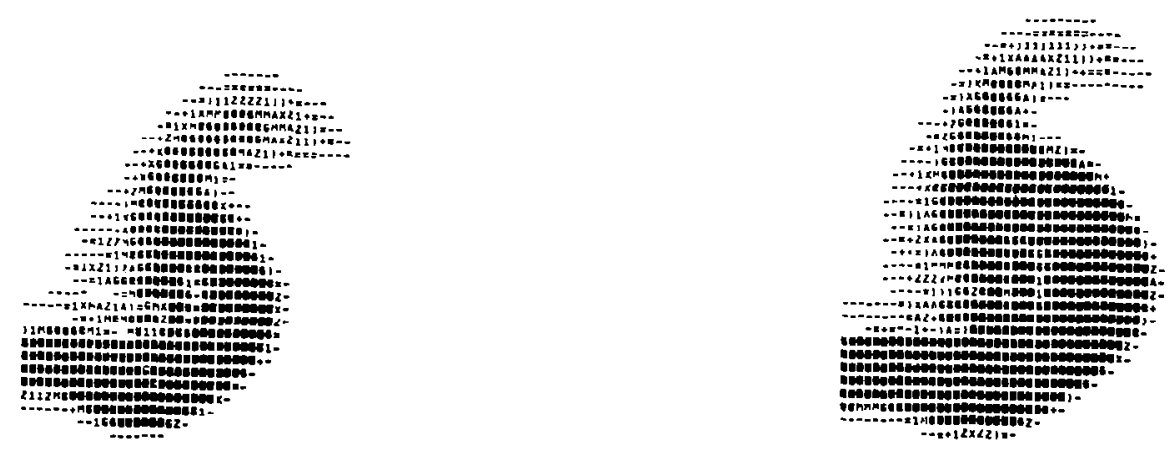

$\begin{aligned} t I_{f^{\prime}} / 2 \pi s^{2} & =0.4 \\ q^{2} / q_{n}^{2} & =27.0\end{aligned}$

$$
\begin{aligned}
t \Gamma_{f^{\prime}} / 2 \pi s^{2} & =0.6 \\
q^{2} / q_{n}^{2} & =44.0
\end{aligned}
$$

Figure 49. Production of turbulent kinetic energy during merging of the vorticity distributions shown in figure 48. The high turbulence levels result from the straining apart of the negative fuselage vortex by the flap vortex $\left[q_{n}^{2}=0.1\left(\Gamma_{f} / 2 \pi s\right)^{2}\right]$ 
The turbulent energy components are equally partitioned and the cross-correlations taken to be zero initially.

Figure 50 shows a comparison of the trailed vorticity in isopleth plot form. The distributions on the left are those given in figure 44 with time converted to downstream distance in wing spans (since lift coefficient and aspect ratio are now specified). The smallest circles carry the largest values, since the plots have been made by nondimensionalizing the vorticity with the maximum value at $x / b=0$. As can be seen, the jet engines do not hinder the merging of flap and tip vortices. At 12 spans downstream, the jet engines give slightly more spreading of the trailed vorticity; hence, the lower maximum value.

Figure 51 shows the intensity of the turbulent kinetic energy. Note that the normalization differs between the run with and without jet exhaust. It is likely that appreciable redistribution of the trailed vorticity is yet to occur owing to the large values of turbulent kinetic energy in the wake. This feature is overemphasized by taking the initial integral scale parameter $\Lambda / \mathrm{s}$ to be 0.2 for both runs. The integral scale parameter in the exhaust jets should be initialized to be somewhat smaller. In any case, the merging phenomenon is not significantly altered by jet exhaust and its accompanying turbulence.

Jet exhaust axial velocity excess plots are shown in figure 52. Note that the inboard engine exhaust, which was introduced into the flap vortex, is slower to decay than the exhaust from the outboard engine, demonstrating the suppression of the production of turbulence by swirl. Engine exhausts are, of course, not the same temperature as the surrounding fluid. The density difference between the exhaust fluid and the atmosphere gives rise through gravity to a buoyancy body force on the exhaust which becomes more significant with wake age. To demonstrate the buoyancy effect, a sample computation was undertaken of four hot jets exhausting into an atmosphere. The engines were positioned as described above. No vortex wake was initially specified so that the generation of vorticity and turbulence by buoyancy could be more easily discerned. A temperature profile was assumed at the exit of each jet which would give rise to a strong buoyancy effect (to check the code):

$$
\frac{\Delta T}{T}=0.5 \exp \left(-52.0 \frac{r^{2}}{s^{2}}\right)
$$

The heat added by this distribution is rather larger than would be added by an existing jet engine. Keeping total thrust equal to the above computation requires

$$
\frac{\Delta U \pi s}{\Gamma_{f}}=14.0 \exp \left(-52.0 \frac{r^{2}}{s^{2}}\right)
$$




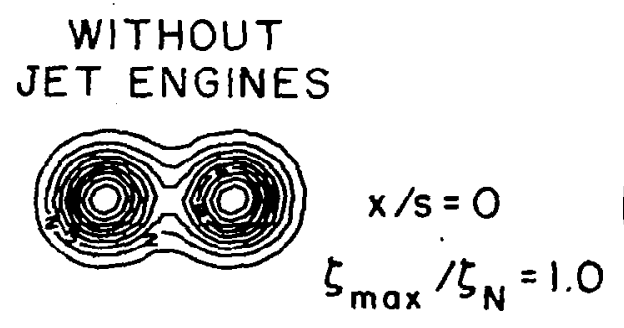

\section{WITH JET} ENGINES
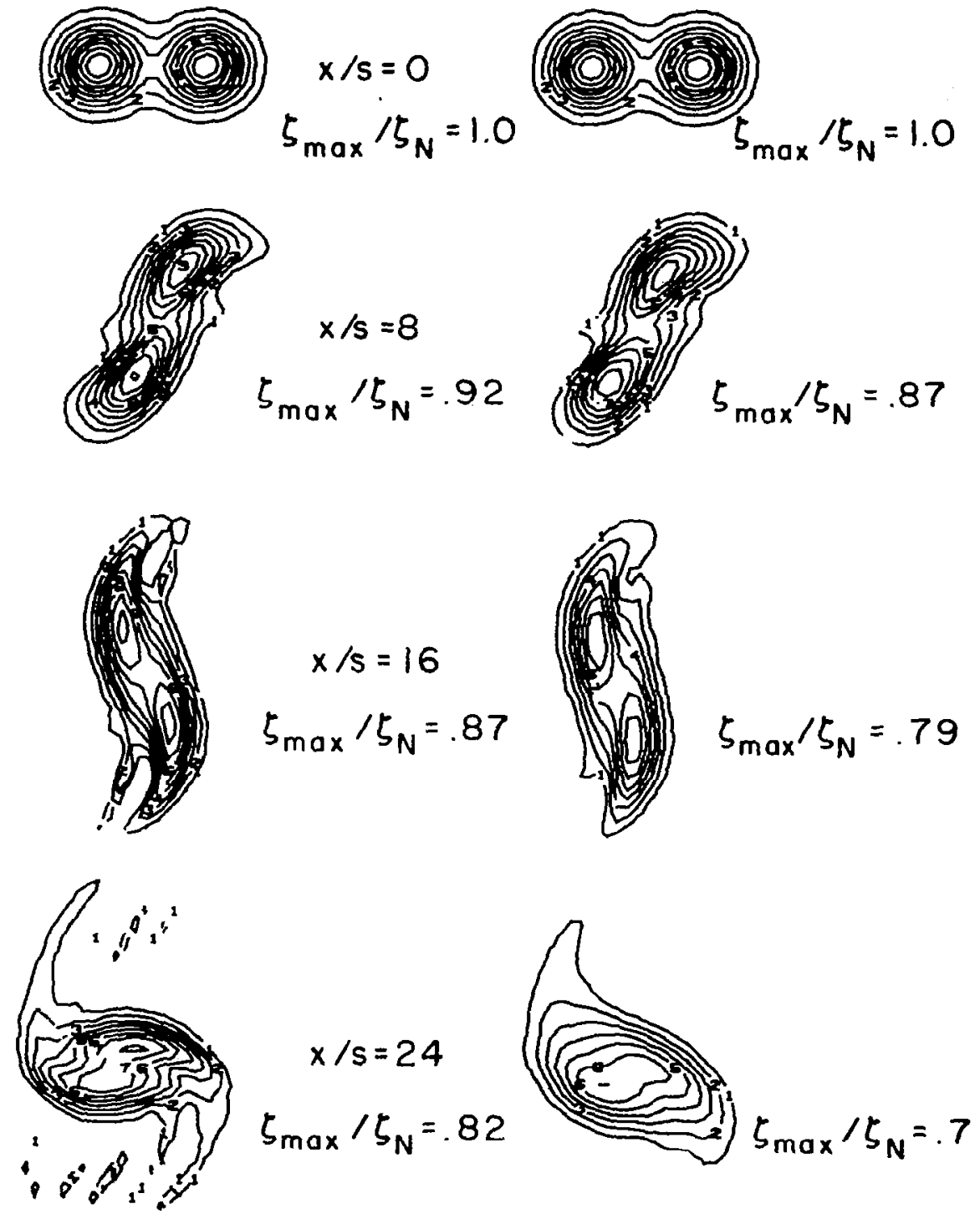

Figure 50. Isopleth plots of the trailed vorticity $\zeta$. $\zeta_{n}=48.5 \Gamma / 2 \pi s^{2}$. 
WITHOUT
JET ENGINES
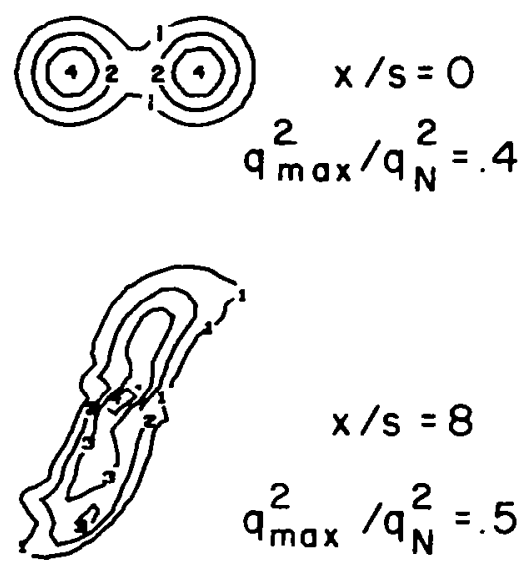

$q_{\max }^{2} / q_{N}^{2}=.5$

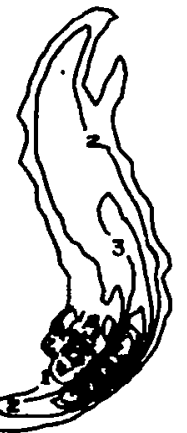

$q_{\max }^{2} / q_{N}^{2}=1.12$
WITH JET

ENGINES

( $\left.\mathrm{C}^{2}, 2\right)$

$q_{\max }^{2} / q_{N}^{2}=.25$
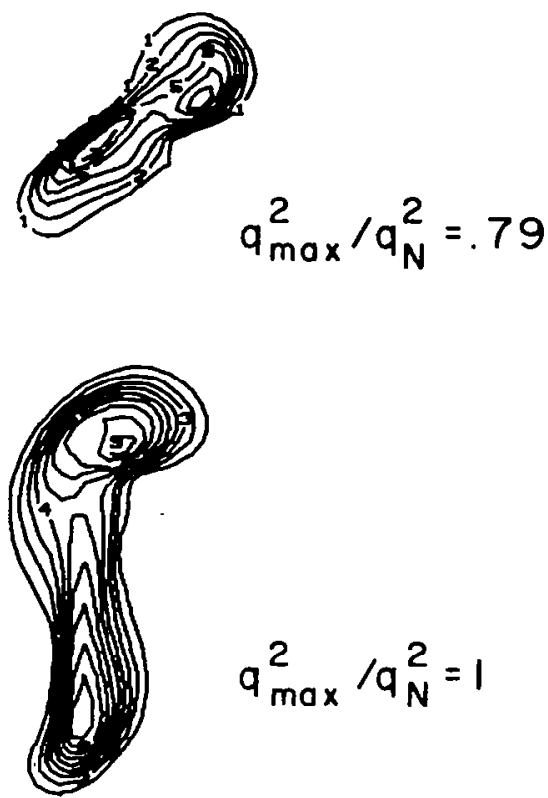

$q_{\max }^{2} / q_{N}^{2}=.82$

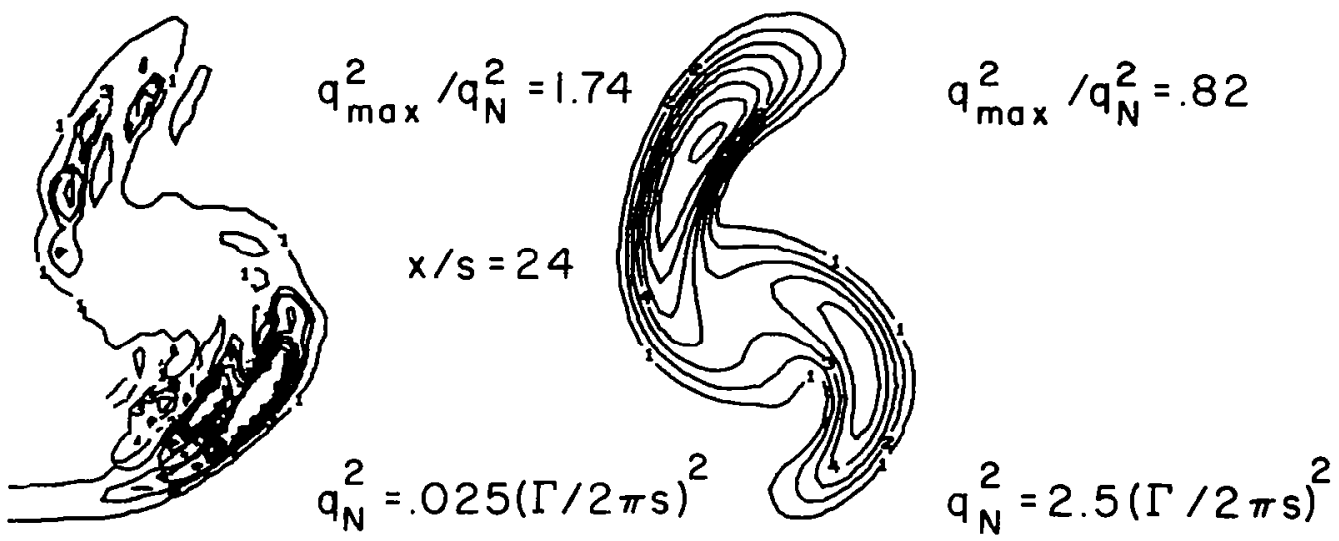

F1gure 51. Isopleth plots of the turbulent kinetic energy. 


\section{WITH JET}

ENGINES

$$
\begin{aligned}
x / s & =0 \\
\Delta U_{\max } / \Delta U_{N} & =1.0
\end{aligned}
$$

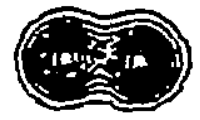

$x / 5=8$

$\Delta U_{\max } / \Delta U_{N}=.72$

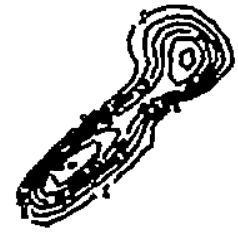

$$
\Delta U_{m a x} / \Delta U_{N}=.45
$$
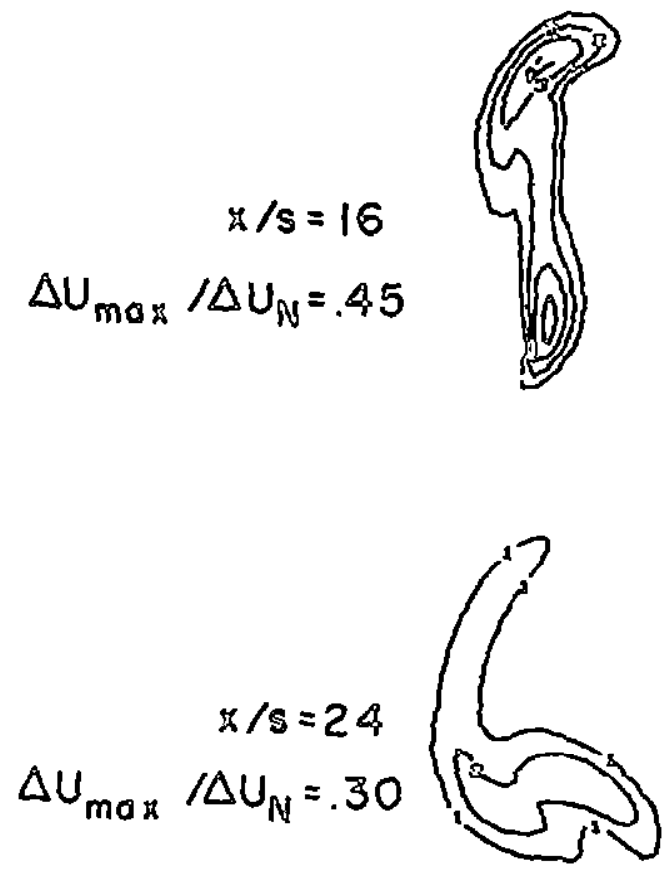

Figure 52. Isopleth plots of the jet exhaust axial velocity excess. $\Delta U_{n}=8.0 r / 2 \pi s$. 
The turbulent kinetic energy distribution about each engine was given by eq. ( 42 ).

The resulting decay of the exhaust velocity is shown in figure 53. Without the presence of the vortex swirl velocity and distribution of axial velocity excess, the decay is quite different from that shown in figure 52. In figures 54 and 55 are shown the distributions of temperature and turbulent kinetic energy. Note that the normalizing value for each distribution in figure 55 is changed to allow intensity resolution over the large change in turbulent kinetic energy. The vertical upward motions of the distribution are evident in figures 53, 54, and 55.

The axial or streamwise vorticity produced by buoyancy is not easily presented in intensity plot form since both positive and negative vorticity are generated (the total circulation is, however, zero). Instead, in figure 56 the maximum and minimum values of vorticity are shown as a function of downstream distances.

While the area integral of the total vorticity generated by buoyancy is zero, the $y$ moment which is associated with the vertical impulse given to the fluid by the body force is not. Since this negative impulse in a neutral atmosphere is ever increasing at some time after aircraft passage, the total impulse in the wake is zero. Estimates of this time have been difficult to make with any certainty, but minutes rather than hours appear to be the correct order of magnitude estimate. While admittedly the amount of engine heat used in the above calculation is larger than that discharged by a conventional by-pass ratio engine, it is easy to suspect that buoyant effects as well as the turbulence produced by the kinetic energy of the jet will explain the sensitivity or wake decay to thrust level settings. 

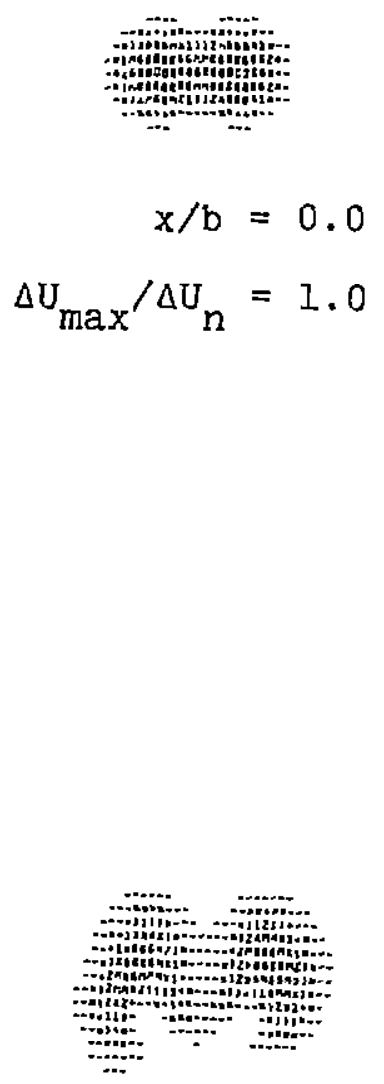

$$
x / b=8.0
$$

$\Delta U_{\max } / \Delta U_{n}=0.57$
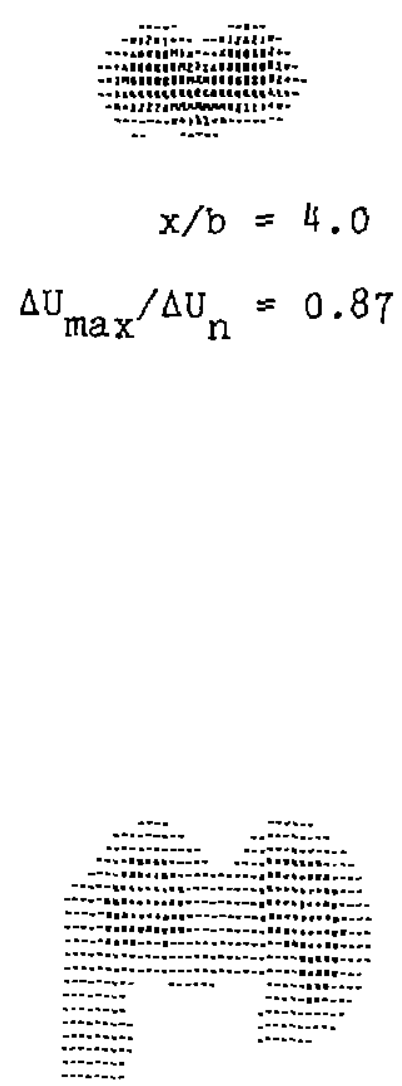

$$
\begin{aligned}
x / b & =12.0 \\
\Delta U_{\max } / \Delta U_{n} & =0.27
\end{aligned}
$$

Figure 53. Intensity plots of the hot jet exhaust axial velocity excess $\Delta U_{n}=14.0 \Gamma_{f} / 2 \pi s$ 

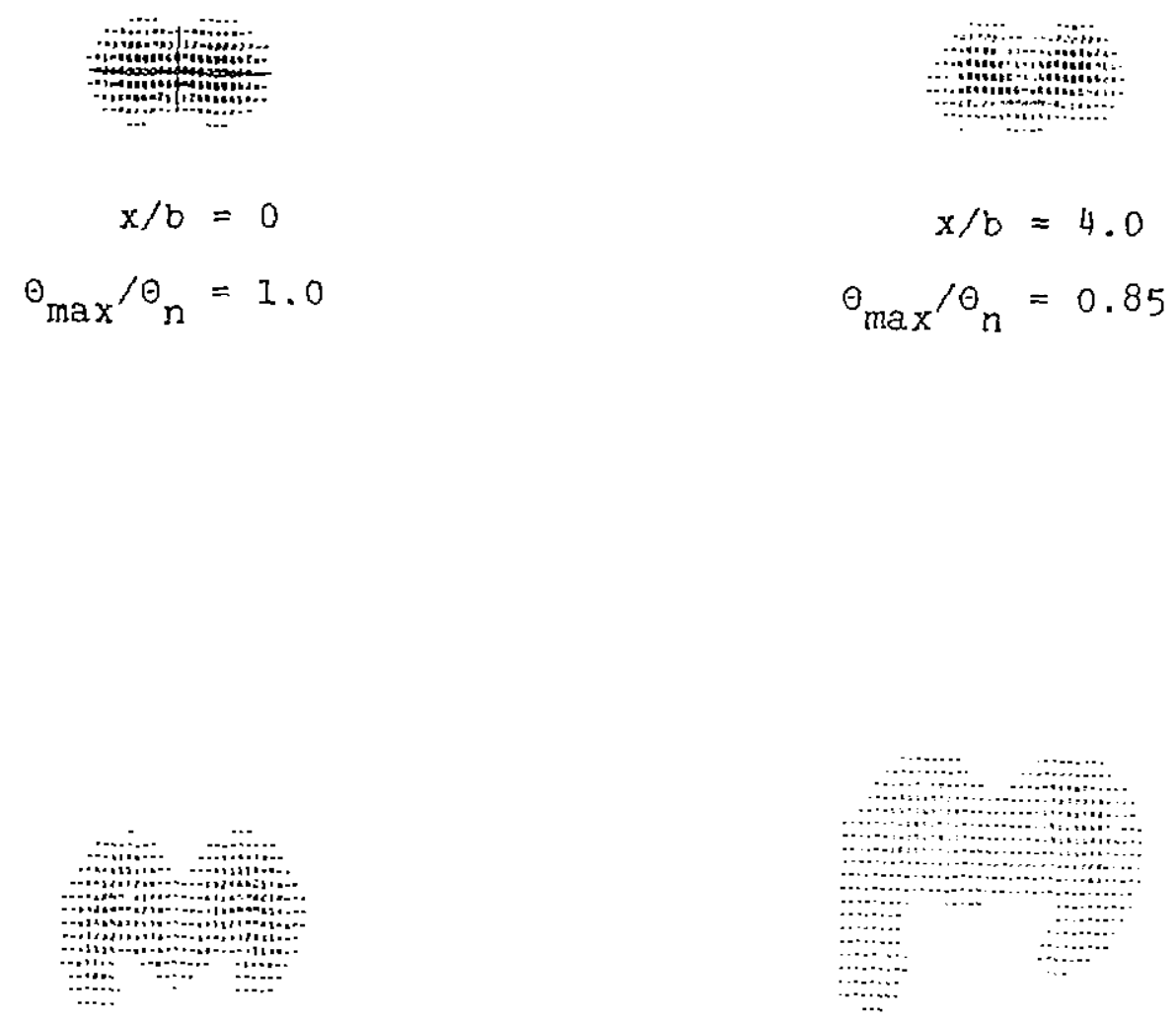

$$
\begin{aligned}
x / b & =8.0 \\
\theta_{\text {max }} / \theta_{n} & =0.49
\end{aligned}
$$

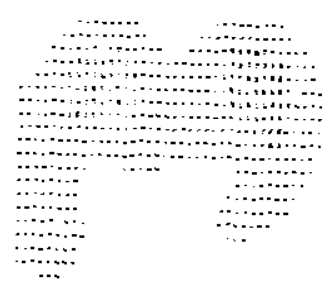

$$
\begin{aligned}
x / b & =12.0 \\
\theta_{\text {max }} / \theta_{n} & =0.23
\end{aligned}
$$

Figure 54. Intensity plots of the exhaust gas temperature corresponding to the axial velocity excess shown in figure $53\left(\theta_{\mathrm{n}} / \theta_{\mathrm{O}}=0.5\right)$ 

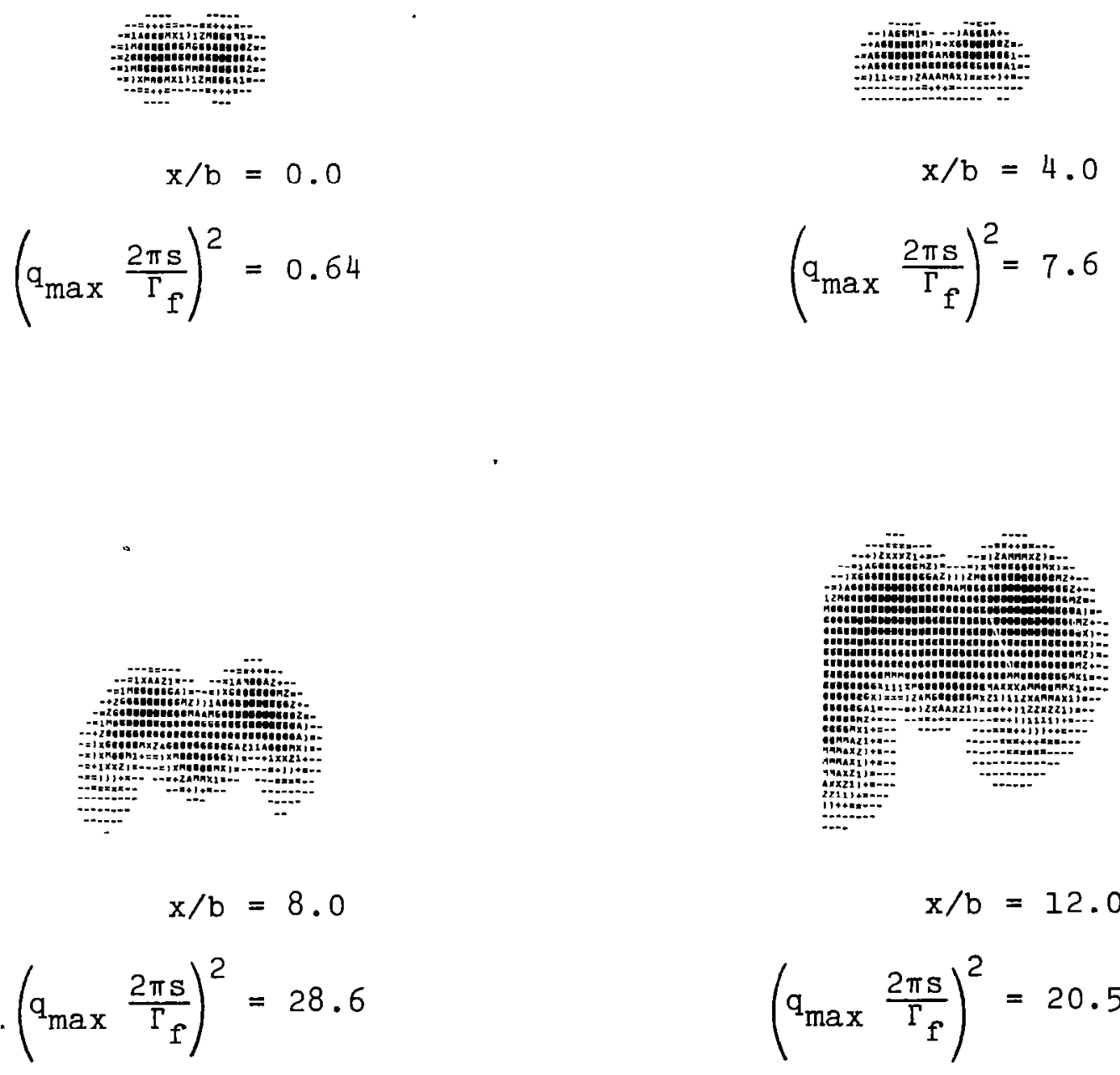

$\begin{aligned} \mathrm{x} / \mathrm{b} & =12.0 \\ \left(\mathrm{q}_{\max } \frac{2 \pi \mathrm{s}}{\Gamma_{\mathrm{f}}}\right)^{2} & =20.5\end{aligned}$

Figure 55. Intensity plots of the turbulent kinetic energy corresponding to the axial velocity excess shown in figure 53 


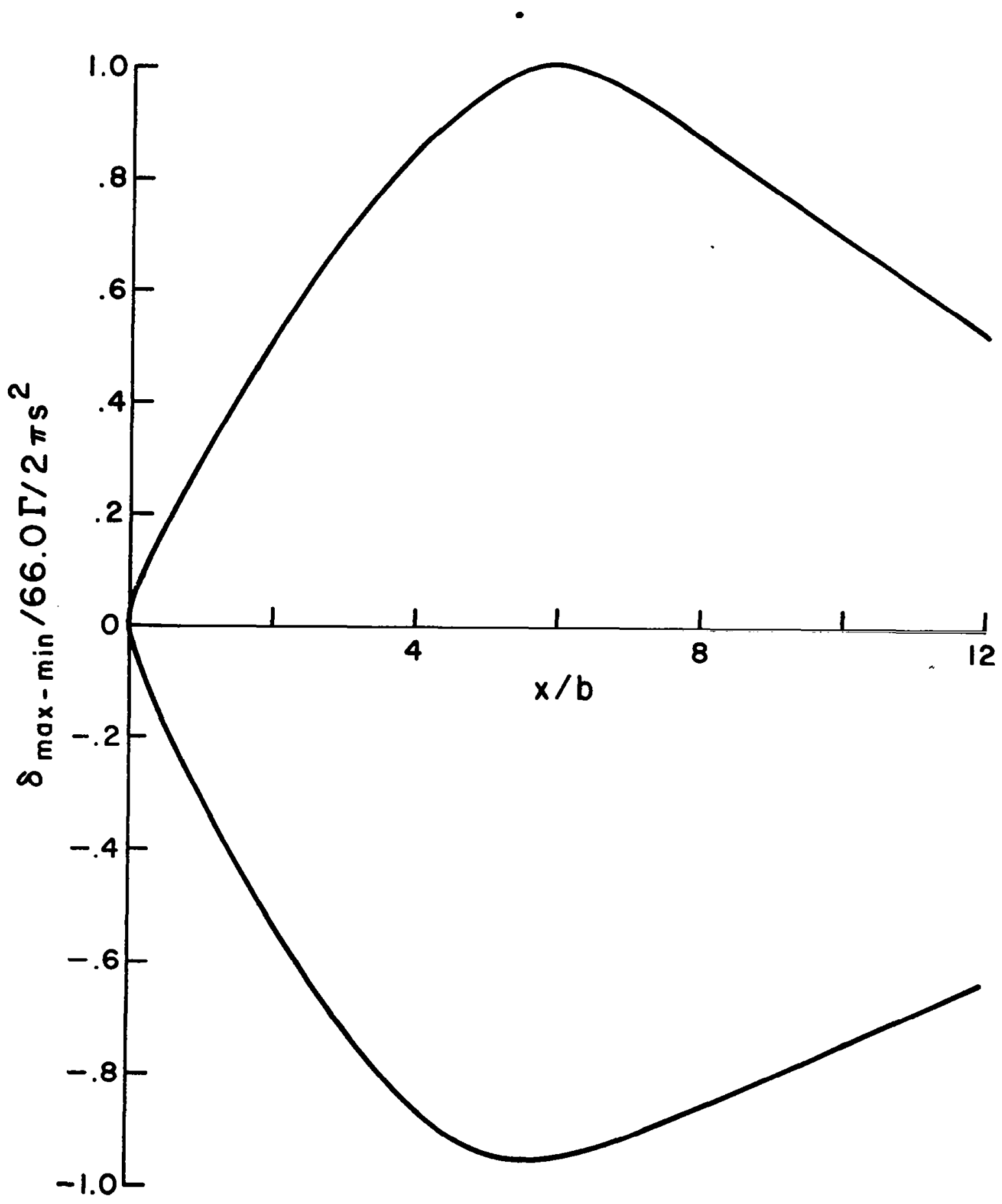

Figure 56. Maximum and minimum axial or streamwise vorticity generated in a buoyant jet exhaust whose axial velocity distribution is shown in figure 57 


\section{CONCLUSIONS AND RECOMMENDATIONS}

Several conclusions from this study can be made with regard to predicting aircraft vortex wake strength and structure.

1. The number, strengths, and inttial positions of vortices trailed from an aircraft wing are determined by the detailed lift distribution on the wing. Prior to this study, only inviscid models were available to predict the swirl velocity distributions in each vortex and the interactions between vortices. The vortex-vortex interaction, called merging, which has been shown in the laboratory, in flight tests, and through analytic techniques to lead to rapid aging of a wake involves the turbulent diffusion of trailed vorticity. A transport model is now available to predict viscous wake interactions.

2. A review of inviscid theories to predict wake structure suggests that the Betz technique is only of limited usefulness in multiple-vortex wakes where strong interactions occur between vortices.

3. Point vortex computations using large numbers of line vortices to represent the initial sheet of trailed vorticity and the subsequent motion of these vortices provide useful information during the initial stages to wake development. This technique, however, excludes the mechanism of viscous diffusion and, therefore, conserves circulation and fluid kinetic energy in the wake halfplane. Extending the model to include the effects of engine exhaust, atmospheric turbulence, and buoyancy is not straightforward.

4. Point vortex computations of the rollup and interactions of the vortices making up the wake of a B-747 aircraft have verifled the trends in terms of induced rolling moments on a following aircraft that have been observed in the laboratory and in flight tests.

5. A study using point vortices has determined that the merging of flap and wing tip vortices can be sensitive to small changes in fuselage vortex strength and position.

6. A study using point vortices has determined that the merging of flap and wing tip vortices can be sensitive to the proximity of the ground.

7. Calculations using the vortex wake transport code developed during this study have shown that a minimum hazard wake is trailed from a generating aircraft when the aircraft configuration is such that a flap and wing tip vortex of the same sign and strength are shed from each wing. To achieve this optimum, the flap vortex is located outboard approximately $40 \%$ of the distance to the tip vortex. The vortices merge, during which convection and diffusion distribute the trailed vorticity widely over the wake. 
8. Calculations using the vortex wake transport code have shown that engine exhausts do not appreciably alter the merging phenomenon. Results suggest that the turbulence added to the wake by engine exhausts help further to diffuse the trailed vorticity prior to, during, and after vortex merging has occurred.

9. The effect of ambient atmospheric turbulence on the aging of an aircraft wake was investigated at constant turbulent dissipation rate. It was shown that under stable atmospheric conditions, when atmospheric macroscale may be less than or equal to the vortex spacing, misleading results may be obtained if vortex aging is correlated only with the turbulent dissipation rate. This result cautions against using one parameter to characterize the ability of the atmosphere to dissipate aircraft wake vortices.

The results obtained from this study warrant the following recommendations.

It is becoming increasingly apparent that the key to being able to explain why small changes in the configuration of an aircraft can lead to a substantially stronger or weaker wake lies, to a large extent, in knowing what has been done to the wing lift distribution. Analytical techniques either do not exist or appear to have difficuly in predicting the lift distribution on a wing with landing gear, flaps, and spoilers deployed. It is recommended that accurate load distributions be obtained experimentally for the B-747 aircraft, particularly for configurations whose wakes have been probed or measured. The load distribution data will permit explanation of existing data showing conflguration sensitivity and will permit minimization techniques to be applied to other aircraft.

Results of studies presented here suggest that the vortex merging interactions in the vicinity of the ground should be the subject of experimental and analytical studies. A minimum hazard configuration cannot be strongly altitude- or aircraft attitudesensitive.

Methods to assess the ability of the atmosphere to dissipate wakes are now available. In light of the fact that measurement of wake decay in a real atmosphere is an enormously difficult task, these methods should now be exploited. 
APPENDIX A.

\section{ANALYTICAL PREDICTION OF MERGING BOUNDARIES}

Moore and Saffman (ref. 8) have given an analysis of a vortex of elliptic cross-section in which the vorticity is uniform and have determined far field flows in which steady, stable solutions are possible. The results of this analysis are extended to determine the proximity $d$ of a vortex of strength $\Gamma_{2}$ to a vortex of initial diameter $d_{c}$ and strength $\Gamma_{1}$ (uniform vorticity) which will not yield a steady solution of elliptical cross-section. The two vortices are shown in the sketch.

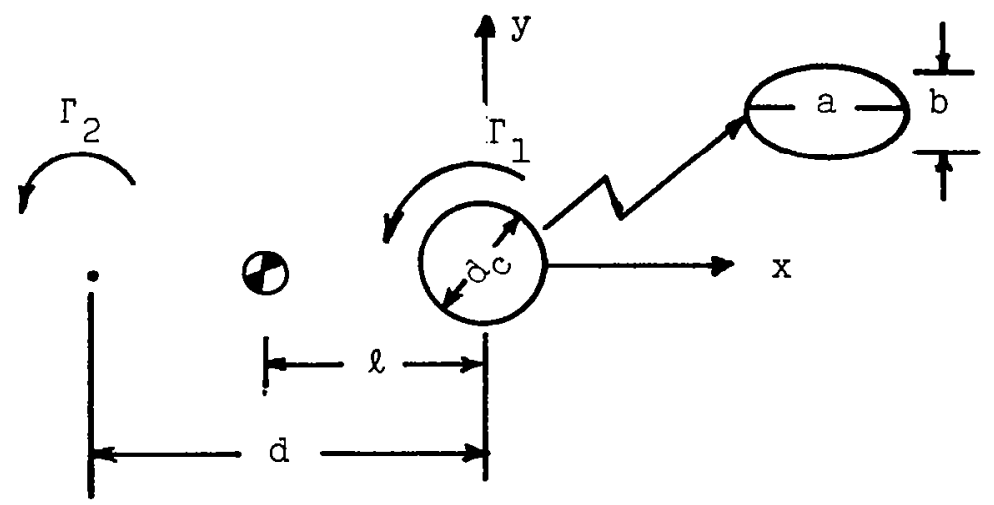

A solid body rotation flow field is added to cancel the rotation. The flow field is then steady. The streamfunction for the solid body flow field is

$$
\psi=\frac{1}{2} \Omega\left[(x-\bar{x})^{2}+y^{2}\right]
$$

where $\Omega=\left(\Gamma_{1}+\Gamma_{2}\right) / 2 \pi d^{2}$ and $\bar{x}$ is the centroid and is given by $\overline{\mathrm{x}}=-\mathrm{d} /\left(1+\Gamma_{1} / \Gamma_{2}\right)$. by

The flow field imposed on the vortex at $x=y=0$ is given

$$
\psi=\frac{\Gamma_{2}}{4 \pi d^{2}}\left(x^{2}-y^{2}\right)+\frac{1}{2} \Omega\left(x^{2}+y^{2}\right)+\theta\left(x^{3} / a^{3}\right)
$$

Substituting into Moore and Saffman's equation (2.16C) with 


$$
e=\frac{\Gamma_{2}}{2 \pi d^{2}}, \gamma=-\Omega, \text { and } \omega_{0}=\frac{2 \Gamma_{l}}{\pi d_{c}^{2}}-2 \Omega
$$

yields

$$
\theta^{3}(s+2)+\theta^{2}\left(-2 s t^{2}+s+2\right)+\theta\left(2 s t^{2}-s\right)-s=0
$$

where $s=\Gamma_{1} / \Gamma_{2}$ and $t=2 \mathrm{~d} / \mathrm{d}_{\mathrm{c}}$. Real positive roots with $\theta=a / b>1$ have the major axis of the ellipse on the $x$-axis $(a / b$ is the major to minor axis ratio). Those roots lying $0<\theta<1$ have the major axis along the y-axis.

The solutions obtained by solving eq. (A.3) must be checked for stability using Moore and Saffman's equation (3.17). The curves along which steady, stable solutions cease to exist in terms of the parameters $\Gamma_{1} / \Gamma_{2}$ and $d / d_{c}$ are shown in figure 3 . 
APPENDIX B.

COMPUTATION OF A MEASURE OF THE ROLLING MOMENT INDUCED

ON A FOLLOWING AIRCRAFT IN THE WAKE OF A B-747.

Discrete Vortex Wake Model

First, the wing load distribution for the aircraft configuration being studied is needed. A standard approach to predict this distribution analytically is vortex lattice theory. The wing is modeled by a lattice of horseshoe vortices whose strengths are such that the flow normal to the mean camber line of the wing at specified points is zero; $1 . e$., no flow through the wing is allowed. The lift associated with each vortex is computed and summed appropriately to determine the spanwise wing lift distribution, total lift, and pitching moment. From the latter two quantities plus the c.g. position, the tail lift required to produce equilibrium flight (no pitch acceleration) is calculated.

Then, a desired number of constant strength line vortices, $2 \mathrm{~N}$, is positioned along the span so as to correspond to the previously calculated wing load distribution, where $N$ is the number of vortices for each half of the wing. (One of the $N$ pairs of vortices may be of lesser strength since the spanwise load is not necessarily divisible by an integer number of incremental loads.) An elliptic load distribution is assumed for the horizontal tail, and the resulting single vortex pair is located laterally at $y= \pm \frac{\pi}{4} \frac{b_{T}}{2}$ where $b_{T}$ is the tail span. The vertical position of this vortex pair is determined by aircraft geometry and angle of attack. The total number of vortex pairs comprising the wake is, thus, $N+I$. The trajectories of these $N+I$ vortex pairs as they convectively interact with each other are calculated in time or, equivalently, in downstream distance (see fig. BI).

With the location of the $N+I$ pairs of vortices given in the cross-flow plane as a function of downstream distance $x$, there is yet the problem of determining how hazardous the wake is to an encountering aircraft. This could be approached by using strip theory to determine the roll torque as a function of aircraft position and orientation relative to the wake. Unfortunately, the unrealistically high velocities near the center of each point vortex make this approach singular whenever a vortex lies on the follower's wingtip. This could be circumvented by postulating a vortex core region, but then the results would be sensitive to the core size chosen. A much simpler approach is to assume that the circulation computed in an area characteristic of the dimension of the encountering aircraft is some measure of hazard. One could then simply survey the wake with a square or circular window with dimension equal to the follower aircraft span and sum the circulation in the window. This approach, while simple, has unrealistic step changes 


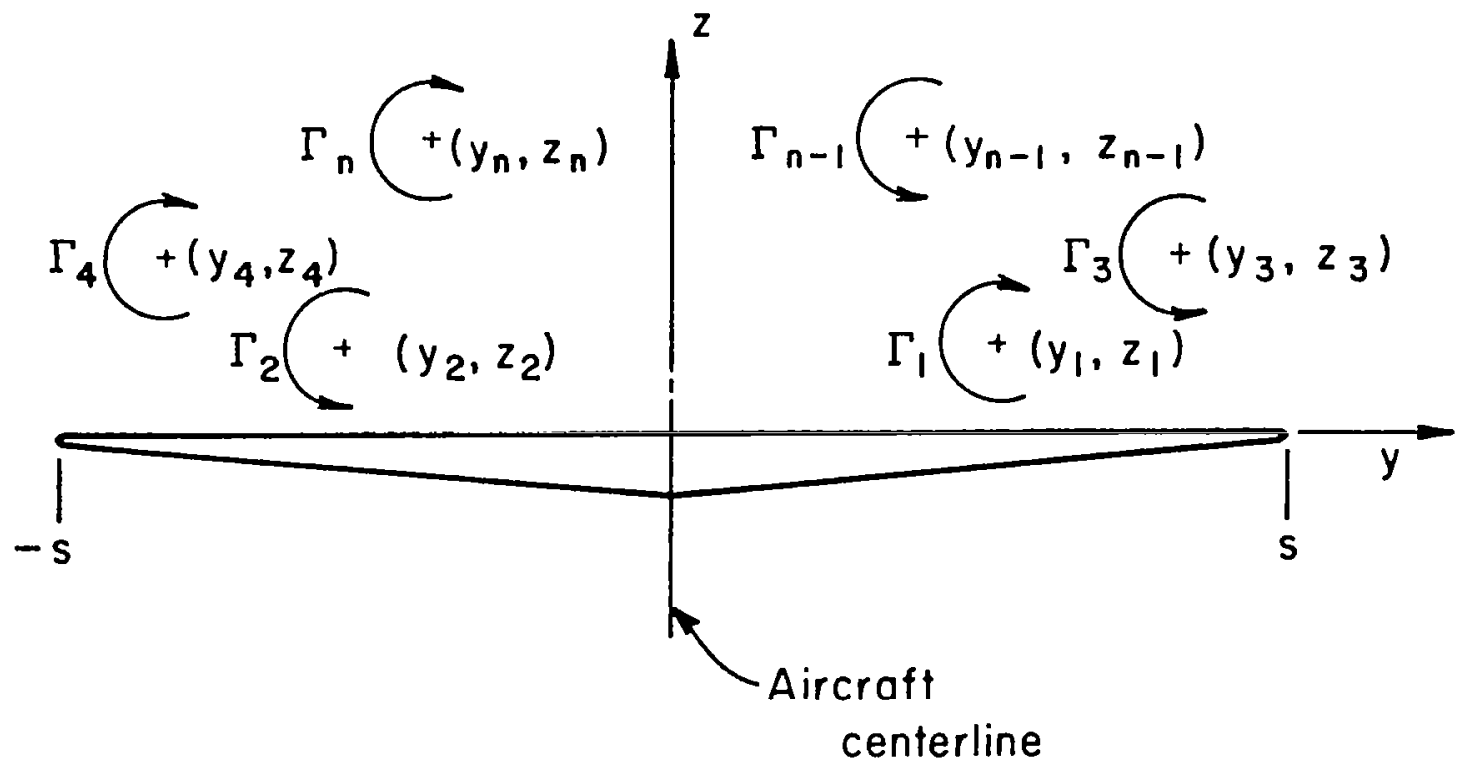

Figure Bl. Geometry for the calculation of the downstream location of vortex centroids. 
as vortex centers cross the boundary of the window. Therefore, a more physically motivated criterion is selected which has no mathematical problem with the singularities at the center of each point vortex. The quantity chosen as the basis for this criterion is the angular momentum within a circle of diameter equal to the wingspan of the follower aircraft. For it is this angular momentum in the fluid surrounding the encountering aircraft that can be transferred to it as a roll torque or rolling moment. Thus, an aircraft roll rate is directly related to the angular momentum of the surrounding fluid.

The mathematical details of these procedures, as depicted in figure 4, to compute the roll hazard in the wake of a generating aircraft are given below. While the method is general, the details are given specifically for the $B-747$ aircraft.

Vortex lattice method for wing load distribution.- The first step in the analysis is to compute wing geometry of the B-747 as a function of trailing edge flap and leading edge slat positions. In particular, the local angles of attack of the mean camber line, $\alpha_{i j}$, must be specified at a series of points on a grid covering the wing.

The grid locations and angles of attack are functions of the basic wing planform, twist, and alrfoil sections, and the positions of the leading edge slat $\dot{s}, \delta_{L E F}$, and the inboard and midspan trailing edge flaps, $\delta_{f_{i}}$ and ${ }^{2} \delta_{f_{m}}$. The wing planform of the

B-747 with and without surfaces extended is shown in figure B2. The mean camber angle at any spanwise location is given by linearly interpolating between the angles given as a function of $x / c$ in Table C.I (Appendix C) where $x / c$ is the chordwise station from the leading edge nondimensionalized by the chord length at that spanwise location. In addition, the outer panel of the wing is twisted with a linear spanwise variation reaching $-3-1 / 2^{\circ}$ at the tip.

$$
\begin{aligned}
& \Delta \alpha_{\text {TWIST }}=.21\left(\mathrm{y}^{\prime}-.71\right) \mathrm{y}^{\prime} \geq .71 \\
& \mathrm{y}^{\prime}=\frac{\mathrm{y}}{\mathrm{b} / 2}
\end{aligned}
$$

The major changes to local angle of attack and planform occur when the flaps and slats are deployed. For the purpose of this study, both devices are assumed to be uncambered surfaces with sealed slots. The surfaces extend from and pivot about a fixed hinge line, thus positioning the endpoint of the surface a horizontal distance $\Delta \mathrm{x}$ and vertical distance $\Delta \mathrm{z}$ from the pivot point as illustrated in flgure B3. Also, in keeping with the accuracy of this analysis, the three leading edge slats are approximated as a single surface with no spanwise variation. 


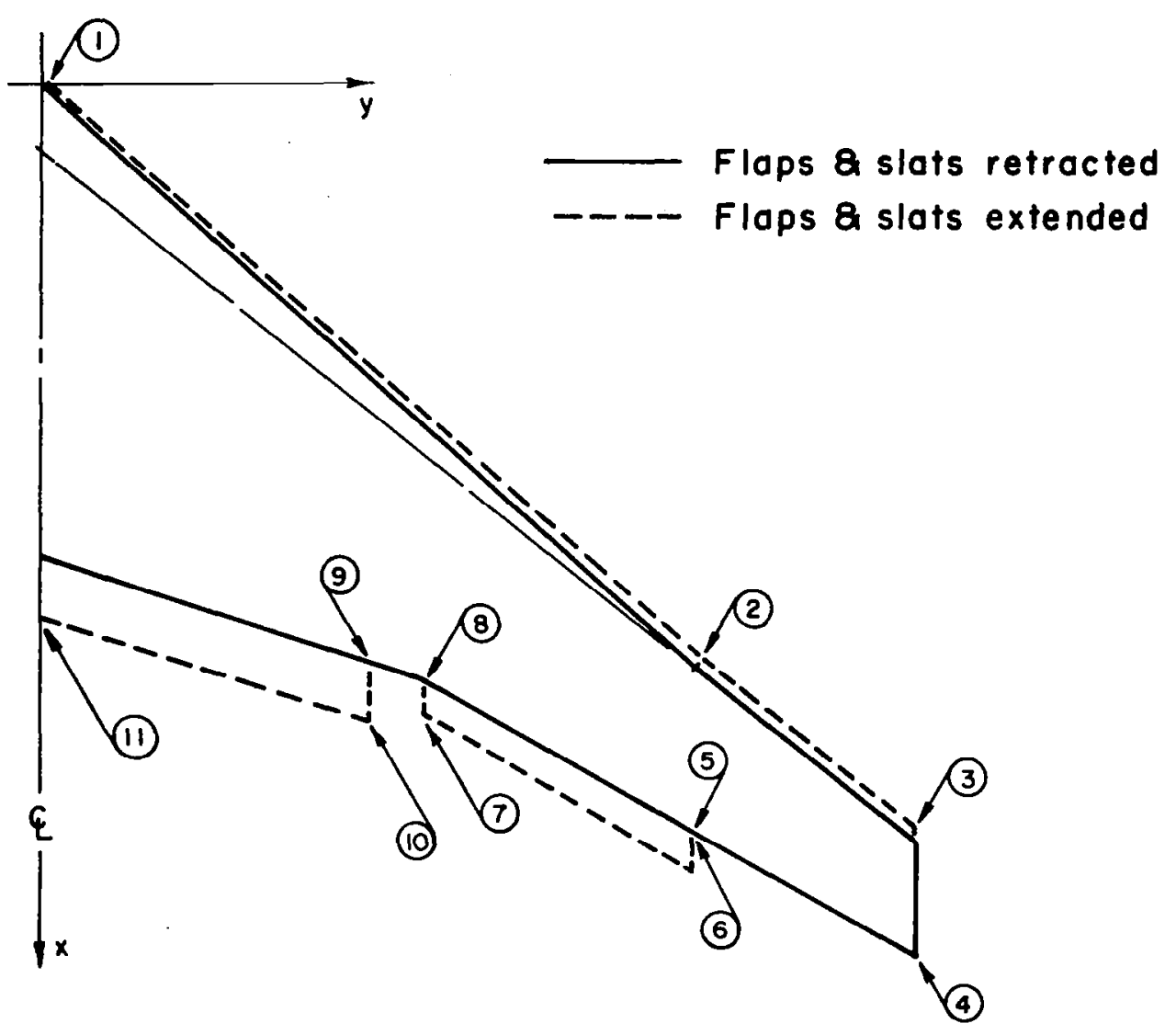

\begin{tabular}{|c|c|c|c|c|}
\hline \multicolumn{5}{|c|}{ PLAN FORM COORDINATES, CM } \\
\hline \multicolumn{5}{|c|}{ FLAP \& SLAT POSITION: } \\
\hline \multicolumn{3}{|c|}{ BOTH RETRACTED } & \multicolumn{2}{|c|}{ BOTHEXTENDED } \\
\hline & $x$ & $y$ & $\mathbf{x}$ & y \\
\hline 1 & 0 & 0 & 45 & 0 \\
\hline 2 & 1925 & 2118 & 1879 & 2118 \\
\hline 3 & 2641 & 2981 & 2595 & 2981 \\
\hline 4 & 3050 & 2981 & 3050 & 2981 \\
\hline 5 & 2545 & 2118 & 2545 & 2118 \\
\hline 6 & 2545 & 2118 & 2677 & 2118 \\
\hline 7 & 2075 & 1308 & 2179 & 1308 \\
\hline 8 & 2075 & 1308 & 2075 & 1308 \\
\hline 9 & 2016 & 1130 & 2016 & 1130 \\
\hline 10 & 2016 & 1130 & 2202 & 1130 \\
\hline 11 & 1656 & 0 & 1838 & 0 \\
\hline
\end{tabular}

Figure B2. B-747 wing planform with and without flaps and slats extended (data supplied by The Boeing Company) 


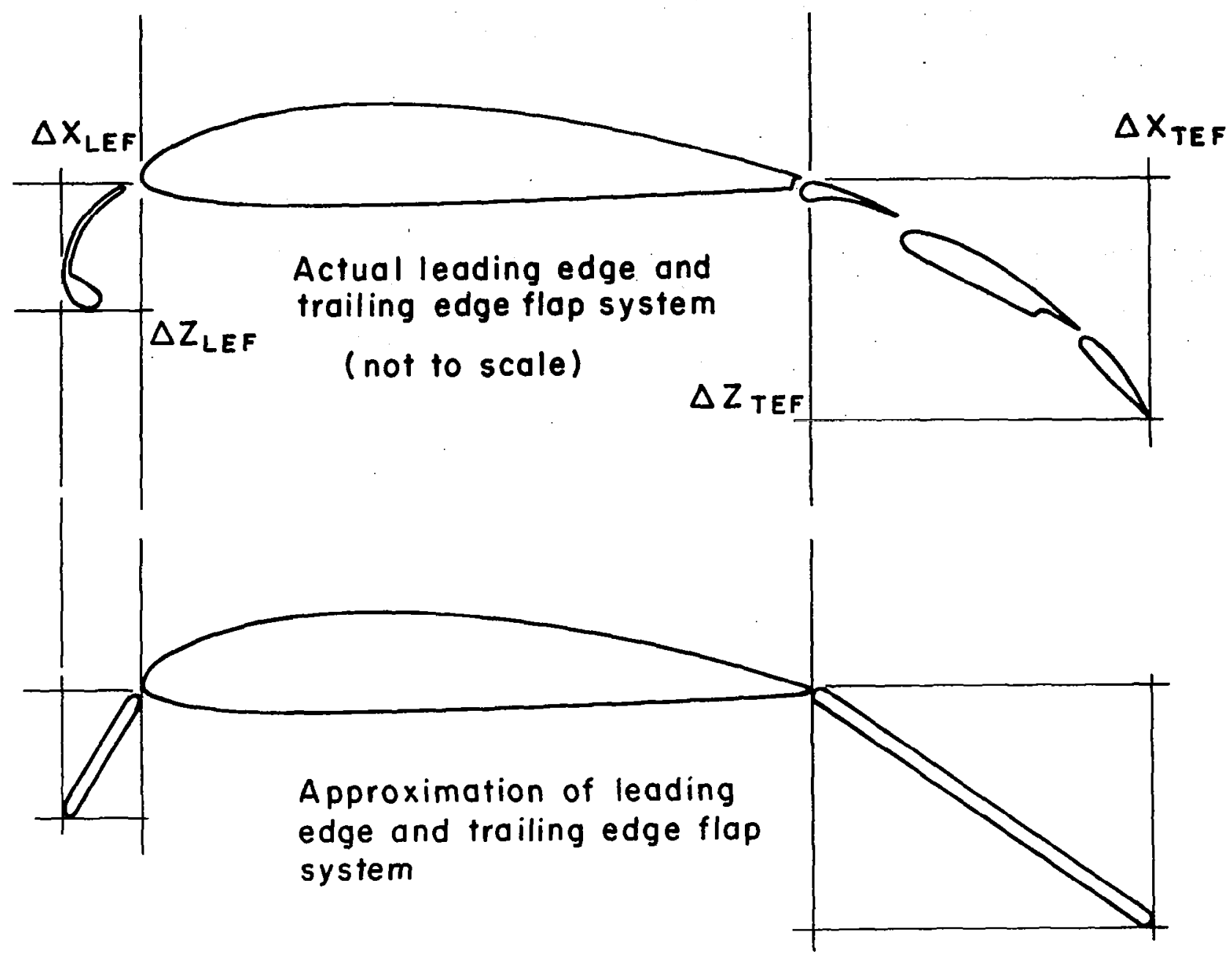

Figure B3. Schemat1c of flap system 
The relations between flap or slat angle setting and these two coordinates $(\Delta x, \Delta z)$ are given in tabular form in Appendix $C$. Subroutines have been developed to interpolate among these data and thereby to specify planform and camber angle changes necessary to predict the wing load distribution.

It then remains to divide the wing into panels, each of which is represented by a horseshoe vortex. Each panel produces a IIft at $1 / 4$ of the panel chord measured from the leading edge, in response to the downwash velocity computed at $3 / 4$ of the paneI chord. A grid containing 180 panels used for this study 1 s shown in figure B4. The number of equal length panels in the chordwise direction is fixed at each spanwise location chosen. The actual length varies with flap and slat deflection.

Thus, for a given set of slat-flap deflections, a mesh of values of local angle of attack is computed and used as input to a modified version of Margason's vortex lattice program (ref. II).

Rather than prescribing a wing Iift coefficient, the program has been modified to accept total lift coefficient and to compute the tail and wing lift contributions. This is done using the equilibrium Ifft and pitching moment equations as shown below.

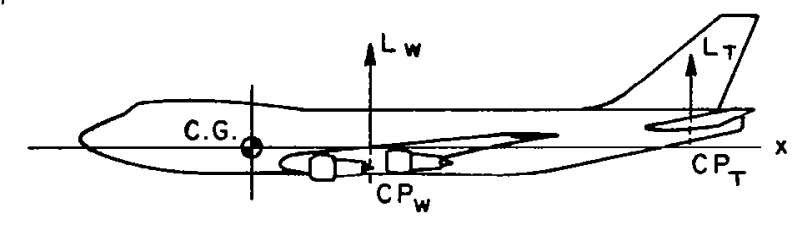

Lift:

$$
\mathrm{C}_{L} \mathrm{qS}_{W}=\mathrm{C}_{L_{W}} \mathrm{qS} \mathrm{S}_{W}+\mathrm{C}_{L_{\mathrm{T}}} \mathrm{qS}_{\mathrm{T}}
$$

Moment about c.g.:

$$
C_{M_{0}} S_{W} c_{W}=C_{L_{W}}{ }^{q} S_{W} c_{W} \frac{x_{C} p_{W}}{c_{W}}-C_{L_{T}}{ } S_{T} c_{W} \frac{x_{C p_{T}}}{{ }^{c} W}
$$

where

$$
\begin{aligned}
C_{L} & =\text { lift coefficient } \\
C_{M_{O}} & =\text { moment coefficient at } C_{L}=0 \\
S & =\text { area } \\
c & =\text { chord } \\
x_{c p} & =\text { location of center of pressure } \\
W & =\text { wing }
\end{aligned}
$$

$$
\mathrm{T}=\mathrm{tail}
$$




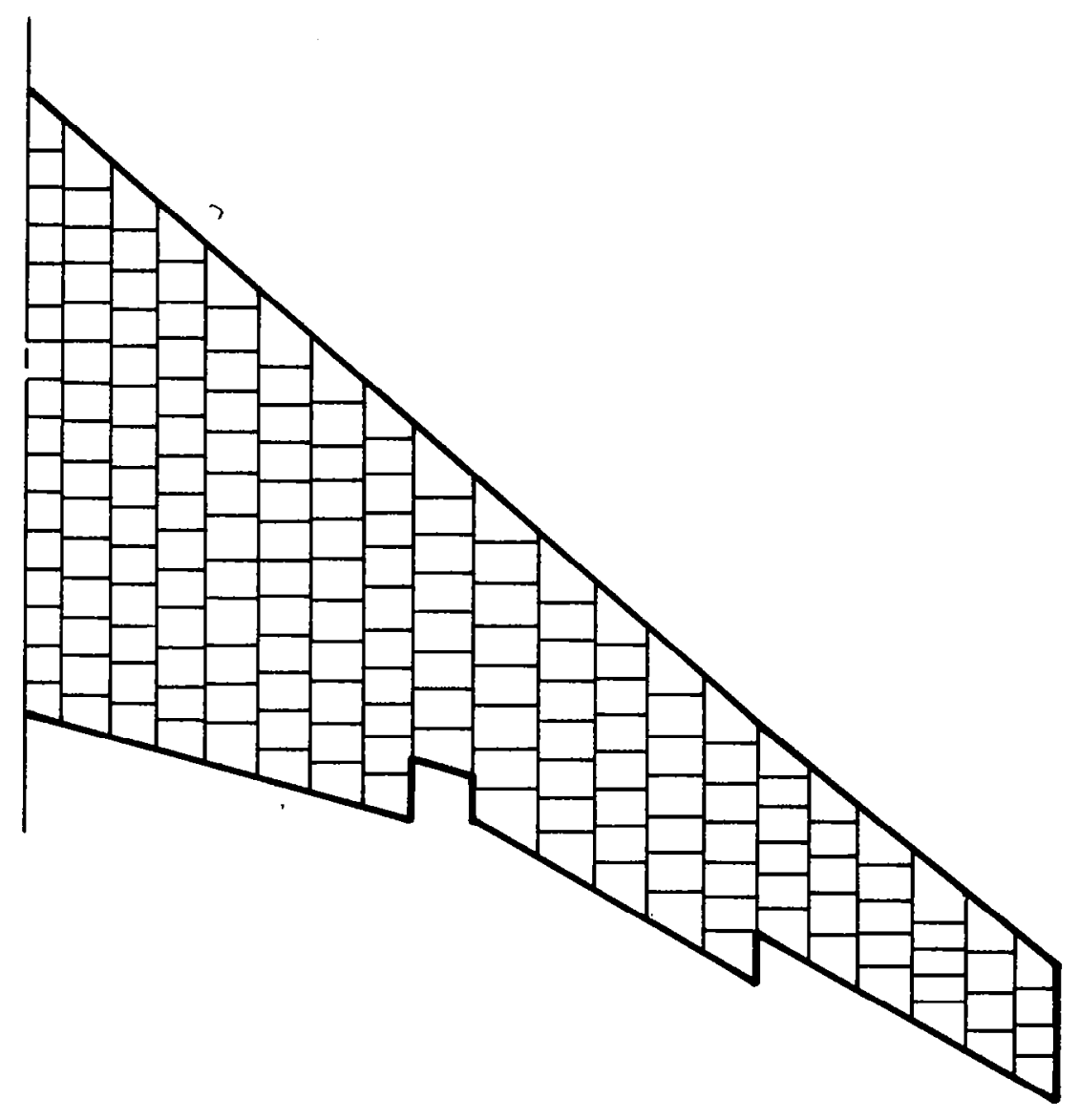

Total panels $=180$ at 20 spanwise stations

(The chordwise dimension of the panels at any spanwise station on the flaps is a function of flap position)

Figure B4. Subdivision of wing into panels for calculation of wing load distribution by vortex lattice theory 
Combining eqs. (B.2) and (B.3) to eliminate $\mathrm{C}_{\mathrm{L}_{\mathrm{T}}}$ yields

$$
\mathrm{C}_{\mathrm{L}_{\mathrm{W}}}=\frac{\mathrm{C}_{\mathrm{L} \mathrm{x}_{\mathrm{cp}}}-\mathrm{C}_{\mathrm{M}_{\mathrm{O}}}{ }^{\mathrm{c}_{W}}}{\mathrm{x}_{\mathrm{C} \mathrm{p}_{\mathrm{T}}}-\mathrm{x}_{\mathrm{c}} \mathrm{p}_{\mathrm{W}}}
$$

For this study, the total lift coefficient is $\mathrm{C}_{\mathrm{L}}=1.2$.

Using a constant lift curve slope, the aircraft angle of attack $\alpha$ is calculated, and the wing load distribution is obtained as

where

$$
\frac{c_{\ell}}{C_{L} \bar{c}_{W}}=f\left(y^{\prime}\right)
$$

$$
\begin{aligned}
c_{\ell} & =\text { sectional lift coefficlent } \\
c & =\text { local chord } \\
c_{L} & =\text { total lift coefficient } \\
\bar{c}_{W} & =\text { reference wing chord }
\end{aligned}
$$

In addition, the tail load magnitude is calculated from eq. (B.2). These data, $\frac{c_{\ell^{c}}}{c_{L^{c}} \bar{c}_{W}}\left(y^{\prime}\right), I_{T}$, and $\alpha$ are then used to generate a point vortex model of the flow field.

"Discretization" of the wing load distribution and computation of the point vortex aircraft wake.- Given the wing load distribution $\left(\frac{c_{l^{c}}}{c_{L} \bar{c}_{W}}\right)_{1}$ at locations $y_{i}, z_{i}$ and the tail load $L_{T}$ at $\mathrm{y}_{\mathrm{T}}, \mathrm{z}_{\mathrm{T}}$, which have been calculated for a particular flap-slat configuration, it is necessary to model the aircraft wake flow field using a finite number $N$ of vortex pairs for the wing and $I$ for the tail. For the computations undertaken here, $30 \leq N \leq 99$, with approximately 40 used most of the time. The discretization of the trailed vortex sheet calculates the locations of vortices of constant strength as follows.

An initial incremental load, $\frac{\Delta c_{\ell}}{C_{L} \dot{\bar{c}}_{W}}=\Delta l_{0}$, is chosen and, starting from the wing tip, the file is checked until a value $\left(\frac{c_{\ell c}}{c_{L} \bar{c}_{W}}\right)_{1} \geq \Delta l_{0}$ is found. The exact location at which $\frac{c_{\ell}{ }^{c}}{C_{L} \bar{c}_{W}}=\Delta l_{O}$ 
is found by interpolation. The search then resumes in the inboard direction until an increase or decrease in load from $n \Delta l_{0}$ (in this case $n=1$ ) of $\Delta l_{0}$ is found. This process is repeated across the semispan of the wing until $y^{\prime}=0$ is reached. At this point, a vortex of reduced strength corresponding to $\left(\frac{c_{\ell} c}{C_{L} \bar{c}_{W}}\right)_{y^{\prime}=0}-n \Delta l_{0}$ is computed at the spanwise location $\mathrm{y}^{\prime}\left(\mathrm{n} \Delta \mathrm{l}_{\mathrm{g}}\right) / 2$. The number of vortices computed by this process is compared with the desired number and, if $\mathrm{N}_{\text {computed }}>\mathrm{N}_{\text {desired, the initial incremental load }}$ is increased by $10 \%, \Delta \ell_{1}=1.1 \Delta l_{0}$, and the process is repeated unt1l $\mathrm{N}_{\text {computed }} \leq \mathrm{N}_{\text {desired }}$.

In addition, the vortex strength associated with the tail load is computed as

$$
\Gamma_{T}=\frac{4 C_{L_{T}} \bar{c}_{T}}{\pi C_{L} \bar{c}_{W}}
$$

The tall loading is assumed elliptical so that

$$
\mathrm{y}_{\mathrm{T}}^{\prime}=\frac{\mathrm{y}_{\mathrm{T}}}{\mathrm{b} / 2}=\frac{\pi \mathrm{b}_{\mathrm{T}}}{4 \mathrm{~b}}
$$

The vertical location of this vortex relative to the wing vortex plane is a function of angle of attack as shown below.

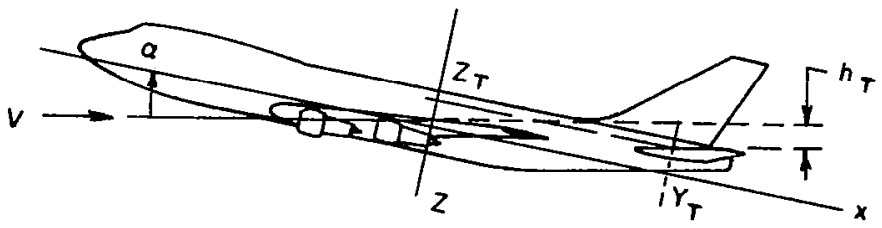

$$
z^{\prime}=\frac{h_{T}}{b / 2}=\frac{z_{T}}{b / 2} \cos \alpha-\frac{x_{T}}{b / 2} \sin \alpha
$$

The wing vortex strengths and initial positions $\Gamma_{i}, y_{i}$, $z_{1}$, where $z_{i}=0$ and, similarly, for the single vortex tail pair $\Gamma_{T}, y_{T}, h_{T}$, are stored, and the calculation of their motion is undértaken. 
The velocity of a point vortex in the wake field is obtained by summing the velocity contributions made by all the other vortices at the center of the vortex in question. This is

$$
\begin{gathered}
\dot{y}_{m}=U \frac{d y_{m}}{d x}=\sum_{\substack{i=1 \\
i \neq m}}^{N+1} \frac{\Gamma_{i}}{2 \pi}\left[\frac{-\left(z_{m}-z_{i}\right)}{\left(z_{m}-z_{i}\right)^{2}+\left(y_{m}-y_{i}\right)^{2}}+\frac{z_{m}-z_{i}}{\left(z_{m}-z_{i}\right)^{2}+\left(y_{m}+y_{i}\right)^{2}}\right] \\
\dot{z}_{m}=U \frac{d z_{m}}{d x}=\sum_{\substack{i=1 \\
i \neq m}}^{\frac{N+1}{2 \pi}}\left[\frac{y_{m}-y_{i}}{\left(z_{m}-z_{i}\right)^{2}+\left(y_{m}-y_{i}\right)^{2}}-\frac{y_{m}+y_{i}}{\left(z_{m}-z_{i}\right)^{2}+\left(y_{m}+y_{i}\right)^{2}}\right] \\
-\frac{\Gamma_{m}}{4 \pi y_{m}}
\end{gathered}
$$

where positive circulation $\Gamma$ following the right-hand rule is represented by a vector in the $+x$ direction. Nondimensionalizing

$$
\begin{aligned}
& \mathrm{x} \text { by } \mathrm{C}_{\mathrm{I}} / \mathrm{b} \pi \mathrm{A} \text { where } \mathrm{A}=\text { wing aspect ratio } \\
& \text { y by } b / 2 \\
& z \text { by } b / 2
\end{aligned}
$$

and substituting for $\Gamma_{i}$

$$
\Gamma_{i}=\left(\frac{c_{\ell}{ }^{C}}{c_{L} \bar{c}_{W}}\right)_{i} \frac{{ }^{U} \bar{c}_{W} C_{L}}{2}
$$

the trajectory equations become:

$$
\frac{d y_{m}^{\prime}}{d x^{\prime}}=\sum_{\substack{i=1 \\ i \neq m}}^{N+1}\left(\frac{c_{\ell} c}{c_{L} \bar{c}_{W}}\right)_{i}\left[\frac{-\left(z_{m}^{\prime}-z_{i}^{\prime}\right)}{\left(z_{m}^{\prime}-z_{i}^{\prime}\right)^{2}+\left(y_{m}^{\prime}-y_{i}^{\prime}\right)^{2}}+\frac{z_{m}^{\prime}-z_{i}^{\prime}}{\left(z_{m}^{\prime}-z_{i}^{\prime}\right)^{2}+\left(y_{m}^{\prime}+y_{i}^{\prime}\right)^{2}}\right]
$$

$$
\frac{d z_{m}^{\prime}}{d x^{\prime}}=\sum_{\substack{i=1 \\ i \neq m}}^{N+1}\left(\frac{c_{\ell} c}{c_{L_{1}} \bar{c}_{W}}\right)\left[\frac{y_{m}^{\prime}-y_{i}^{\prime}}{\left(z_{m}^{\prime}-z_{i}^{\prime}\right)^{2}+\left(y_{m}^{\prime}-y_{i}^{\prime}\right)^{2}}-\frac{y_{m}^{\prime}+y_{i}^{\prime}}{\left(z_{m}^{\prime}-z_{i}^{\prime}\right)^{2}+\left(y_{m}^{\prime}+y_{i}^{\prime}\right)^{2}}\right]
$$




$$
-\frac{\frac{c_{\ell}{ }^{C}}{C_{L} \bar{c}_{W} m}}{2 y_{m}^{\prime}}
$$

These $2 \mathrm{~N}+2$ first-order differential equations are integrated using a modified Runge-Kutta procedure, and the locations $y_{i}^{\prime}$, $z_{i}^{\prime}$ of each vortex saved at specified values of $x^{\prime}$

In addition to specifying the numerical procedure used, a word is in order about the integration step size versus accuracy. Since the system of equations is large, it is particularly necessary to use a small enough step size to track the motion accurately but not small enough to incur significant roundoff errors. Fortunately, the physics of the process requires that the impulse and kinetic energy associated with the relative position of the vortices (Kirchhoff-Routh path function (ref. 2)) in the wake model remain constant. Therefore, these quantities can be monitored to determine if, and check when, the integration process is significantly in error. In general, however, the integration process could be carried out to a nondimensional downstream distance of unity (about $1.5 \mathrm{~km}$ for the B-747) with only a few percent change in the invariants.

This completes the procedure for defining the aircraft wake flow field in terms of trajectories of inviscid point vortices. The remaining step is to evaluate how hazardous the flow field is for smaller following aircraft. 


\section{APPENDIX C.}

TABULAR DATA FOR BASIC WING CAMBER AND FOR PLANFORM AND CAMBER CHANGES DUE TO FLAP EXTENSION

The manufacturer supplied mean camber data for the wing with no flap extensions, as given below, as a function of nondimensional chordwise position $\mathrm{x} / \mathrm{c}$. The characteristic camber is the same for all spanwise locations.

$\begin{array}{ll}\mathrm{x} / \mathrm{c} & \begin{array}{l}\alpha_{\text {Iocal }} \\ \text { (rad) }\end{array} \\ 0 & -.2472 \\ .025 & -.2034 \\ .050 & -.1628 \\ .075 & -.1259 \\ .10 & -.0926 \\ .15 & -.0381 \\ .20 & .0001 \\ .25 & .02168 \\ 1.00 & .0270\end{array}$

TABLE C.I 
TABLE C.2

\section{Leading Edge Flaps}

$\begin{array}{ccc}\delta_{\text {LEF }}(\mathrm{deg}) & \Delta \mathrm{x}(\mathrm{cm}) & \Delta \mathrm{z}(\mathrm{cm}) \\ 0 & 0 & 0 \\ 60 & 46.48 & 66.04 \\ & & \text { Trailing Edge Flaps }\end{array}$

Inboard Flap

$\begin{array}{ccc}\delta_{i}(\mathrm{deg}) & \Delta \mathrm{x}(\mathrm{cm}) & \Delta z(\mathrm{~cm}) \\ 0 & 0 & 0 \\ 2.5 & 108.71 & 14.98 \\ 5.0 & 129.28 & 30.22 \\ 18.0 & 154.94 & 81.13 \\ 25.5 & 161.54 & 208.21 \\ 28.67 & 184.91 & 234.18\end{array}$

Midspan Flap

$\delta_{0}$

0

2.5

5.0

8.5

14.75

19.25

23.0
$\Delta \mathrm{x} .63$

0

61.36

93.26

99.87

109.52

120.09

124.15
$\Delta z .63$

0

10.46

20.62

31.29

55.77

95.19

137.36
$\Delta \mathrm{x} .56$

0

61.26

93.37

99.77

108.71

110.94

111.76
$\Delta \mathrm{z} .56$ 0

11.68

23.06

35.30

62.99

107.44

155.19 


\section{APPENDIX D.}

NUMERICAL BOUNDARY CONDITIONS FOR A FLUID OF INFINITE EXTENT

A new technique is devised to specify computational boundary conditions when the vorticity is not extensively distributed throughout an infinite two-dimensional fluid. Consider the vorticity distribution sketched below at time $t$.

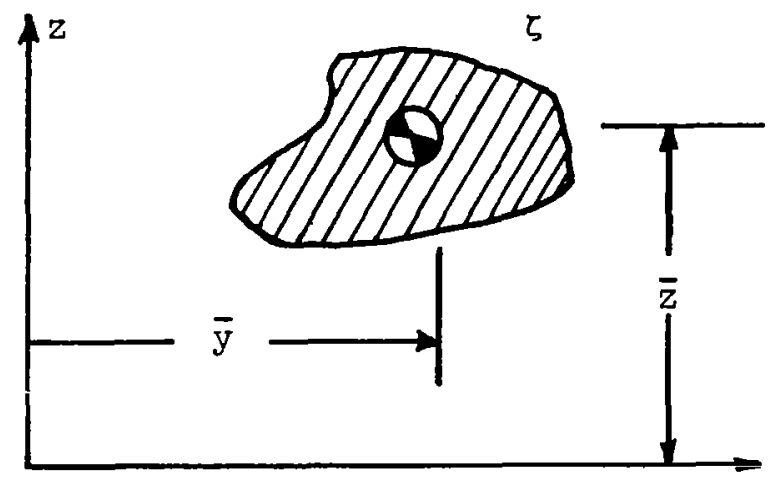

The fluid is incompressible and the motion two-dimensional. At time $t$ the stream function $\psi$ is computed from a Poisson equation

$$
\nabla^{2} \psi=-\zeta(y, z, t)
$$

The solution to (D.I) is

$$
\psi(y, z, t)=-\frac{1}{4 \pi} \int \omega\left(y^{\prime}, z^{\prime}, t\right) \log \left(\left(y-y^{\prime}\right)^{2}+\left(z-z^{\prime}\right)^{2}\right) d A^{\prime}
$$

where the logarithm is the appropriate Green's function.

The $V$ and $W$ velocities are obtained from

$$
\begin{aligned}
& W=-\frac{\partial \psi}{\partial y} \\
& V=+\frac{\partial \psi}{\partial z}
\end{aligned}
$$


Now, in principle, the integral in eq. (D.2) could be carried out numerically and the velocities could be obtained at the computational grid boundaries at time $t$. If the computational grid contained NM points, on the order of $2(N+M)$ integrations over the computational domain would need to be carried out. However, the computer time required would be prohibitive. Therefore, an alternate approach is necessary.

If we $f i x$ our numerical computational boundaries so that they are well away from and surround the region containing vorticity, eq. (D.2) may be approximated by expanding for $y>y^{\prime}$ and $z>z^{\prime}$. The result is

$$
\text { Where } \begin{aligned}
I_{m n} & =\int_{-\infty}^{\infty} y^{\prime n},^{m} \zeta\left(y^{\prime}, z^{\prime}, t\right) d A \\
G_{00} & =10 g r^{2} \\
G_{01} & =-\frac{2 y}{r^{2}} \sum_{n=0}^{\infty} I_{m n} G_{m n} \\
G_{10} & =-\frac{2 z}{r^{2}} \\
G_{02} & =\frac{1}{r^{2}}\left(1-\frac{2 y^{2}}{r^{2}}\right) \\
G_{11} & =-\frac{4 y z}{r^{4}} \\
G_{20} & =\frac{1}{r^{2}}\left(1-\frac{2 z^{2}}{r^{2}}\right)
\end{aligned}
$$

The integers $\mathrm{n}$ and $\mathrm{m}$ are sufficiently large to give the desired resolution of the far field. Our work suggests that $n$ and $m$ need be no larger than 2. The velocity boundary conditions computed from the vorticity at time $t$ are used at $t i m e ~ t+\Delta t$, giving first-order accuracy. One iteration could be used to raise the boundary condition to second-order accuracy, but this has proven to be unnecessary in the Vortex Wake code at this time.

Some additional economy can be gained by suitably choosing the origin of the $y, z$ system to coincide with the centroid of the vorticity distribution. The centroid is defined by 


$$
\begin{aligned}
& \bar{y}=I_{0 I} / I_{00} \\
& \bar{z}=I_{10} / I_{00}
\end{aligned}
$$

which, obviously, is meaningful only if $I_{00} \neq 0$. When this is done, the velocity decays as $r^{-1}$ where $r$ is measured from the centroid. The next term decays as $r^{-3}$.

When the wake of an aircraft is computed, a half-plane calculation can be used. In this case, an image system is used to obtain the induced field from the reflected plane as shown below.

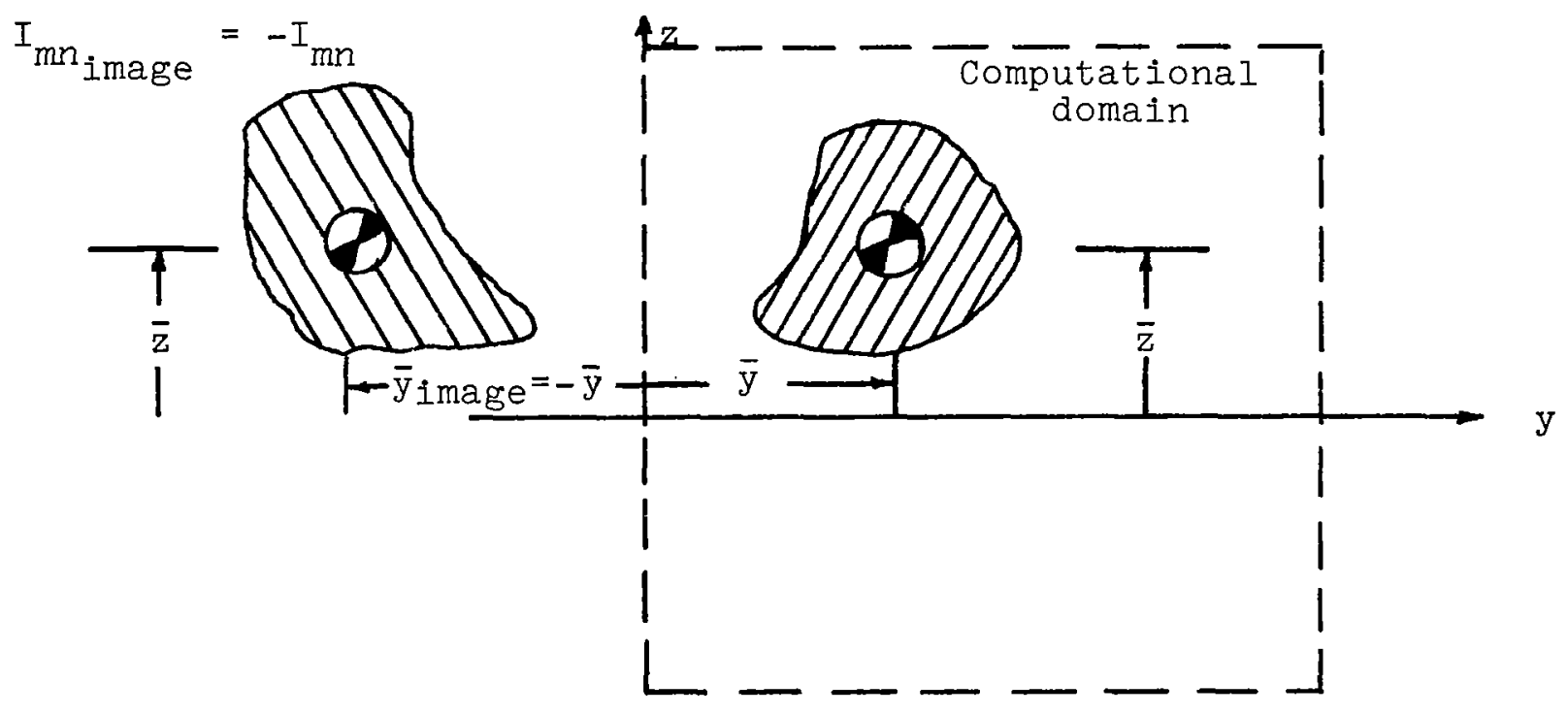

The details of the superposition of velocities are straightforward. 


\section{REFERENCES}

1. Betz, A.: Behavior of Vortex Systems. NACA TM 713 (trans. from ZAMM, Vol. XII.3, 1932).

2. Donaldson, Coleman duP. and Bilanin, Alan J.: Vortex Wakes of Conventional Aircraft. AGARDograph No. 204, May 1975.

3. Donaldson, Coleman duP., Snedeker, Richard S., and Sullivan, Roger D.: A Method of Calculating Alrcraft Wake Velocity Profiles and Comparison with Full-Scale Experimental Measurements. J. Aircraft, vol. 11, no. 9, September 1974, pp. 547555.

4. Snedeker, Richard S. and Bilanin, Alan J.: Analysis of the Vortex Wakes of the Boeing 727, Lockheed L-I01I, McDonneI1 Douglas DC-I0, and Boeing 747 Aircraft. A.R.A.P. Report No. 245, July 1975.

5. Corsiglia, Victor R. and Dunham, R. Earl: Aircraft WakeVortex Minimization by Use of Flaps. Proc. NASA Symposium on Wake Vortex Minimization, 25-26 February 1976, pp. 303-336.

6. Corsiglia, Victor R., Rossow, Vernon J., and Ciffone, Donald L.: Experimental Study of the Effect of Span Loading on Aircraft Wakes. AIAA Paper No. 75-885, presented at AIAA 8th Fluid and Plasma Dynamies Conference, Hartford, 1975.

7. Bilanin, Alan J., Snedeker, Richard S, and Teske, Milton E.: Interactions and Merging Between Line Vortices. A.R.A.P. Report No. 276, March 1976.

8. Moore, D.W. and Saffman, P.G.: Structure of a Line Vortex in an Imposed Strain. In Aircraft Wake Turbulence and Its Detection ( $\mathrm{J} .01$ sen, A. Goldburg, and M. Rogers, eds.), Plenum Press, New York, 1971.

9. Rossow, Vernon J.: Inviscid Modeling of Aircraft Trailing Vortices. Proc. NASA Symposium on Wake Vortex Minimization, 25-26 February 1976, pp. 4-54.

10. Roberts, K.V. and Christiansen, J.P.: Topics in Computational Fluid Mechanics. Computer Physics Comm., vol. 3 supplement, 1972, pp. 14-32.

11. Margason, Richard J. and Lamar, John E.: Vortex-Lattice Fortran Program for Estimating Subsonic Aerodynamic Characteristics of Complex Planforms. NASA TN D-6142, February 1971.

12. Ciffone, Donald L. and Lonzo, C.: Flow Visualization of Vortex Interactions in Multiple Vortex Wakes Behind Aircraft. NASA TM X-62,459, 1975 (see also Vortex Interactions in Multiple Vortex Wakes Behind Aircraft, AIAA Paper No. 76-62, presented at 14th AIAA Aerospace Sciences Meeting, January 1976). 
13. Donaldson, Coleman duP., Sullivan, Roger D., and Rosenbaum, Harold: A Theoretical Study of the Generation of Atmospheric Clear Air Turbulence. AIAA Journal, vol. 10, no. 2, February 1972, pp. 181-187.

14. Lewellen, W. Stephen, Teske, Milton E., and Donaldson, Coleman duP.: Turbulent Wakes in a Stratified Fluid. A.R.A.P. Report No. 226, Part I, September 1974.

15. Lewellen, W. Stephen, Teske, Milton E., and Donaldson, Coleman duP.: Turbulence Model of Diurnal Variations in the Planetary Boundary Layer. Proc. 1974 Heat Transfer and Fluid Mechanics Institute (L.R.Davis and R.E.Wilson, eds.), Stanford University Press, Stanford, 1974, pp. 301-319.

16. Donaldson, Coleman duP.: Atmospheric Turbulence and the Dispersal of Atmospheric Pollutants. Environmental Protection Agency Report No. EPA-R4-73-016a, March 1973.

17. Lewellen, W. Stephen and Teske, Milton E.: Turbulence Modeling and Its Application to Atmospheric Diffusion. Part II. Critical Review of the Use of Invariant Modeling. Environmental Protection Agency Report No. EPA-600/4-75-016b, December 1975.

18. Phillips, O.M.: The Dynamics of the Upper Ocean. Cambridge University Press, Cambridge, 1969.

19. Lumley, John L. and Khajeh-Nouri, B.: Computational Modeling of Turbulent Transport. Advances in Geophysics, vol. 18A, Academic Press, New York, 1974.

20. Mellor, George L. and Yamada, T.: A Hierarchy of Turbulence Closure Models for Planetary Boundary Layers. J. Atmospheric Sciences, vol. 31, 1974, pp. 1791-1806.

21. Rotta, J.C.: Statistiche Theorie nichthomogener Turbulenz. Z. Physik, vol. 129, 1951, pp. 547-572.

22. Lewellen, W. Stephen and Teske, Milton E.: Prediction of the Monin-Obukhov Similarity Functions from an Invariant Model of Turbulence. J. Atmospheric Sciences, vol. 30, 1973, pp. 13401345.

23. Lewellen, W. Stephen, Teske, Milton E., Contiliano, Ross M., Hilst, Glenn R., and Donaldson, Coleman duP.: Invariant Modeling of Turbulent Diffusion in the Planetary Boundary Layer. Environmental Protection Agency Report No. EPA-650/474-035, September 1974 .

24. Lewellen, W. Stephen, Teske, Milton E., and Donaldson, Coleman duP.: Application of Turbulent Model Equations to Axisymmetric Wakes. AIAA Journal, vol. 12, no. 5, May 1974, pp. 620-625.

25. Businger, J.A., Wyngaard, J.C., Izumi, Y., and Bradley, E.F.: Flux-Profile Relationships in the Atmospheric Surface Layer. J. Atmospheric Sclences, vol. 28, 1971, pp. 181-189. 
26. Teske, Milton E.: Vortex Interactions and Decay In Aircraft Wakes, Part II: The Vortex Wake Computer Program User Manual. A.R.A.P. Report No. 271, Vol. II, March 1976.

27. --- Part III. The Vortex Wake Computer Program Programmer Manual. A.R.A.P. Report No. 271, Vo1. III, March 1976.

28. Sullivan, Roger D.: A Program to Compute the Behavior of a Three-Dimensional Turbulent Vortex. Aerospace Research Labs. Report No. ARL-TR-74-0009, December 1973.

29. Buneman, 0.: A Compact Non-Iterative Poisson Solver. Stanford University Institute for Plasma Research Report No. SUIPR 294, May 1969.

30. Carnahan, B., Luther, H.A. and Wilkes, J.0.: Applied Numerical Methods. John Wiley \& Sons, New York, 1969, pp. 452-453, 508 .

31. Tombach, I: Observations of Atmospheric Effects of Vortex Wake Behavior. J. Aircraft, vol. 10, no. 11, November 1973, pp. 641-647.

32. Lewellen, W. Stephen and Williamson, Guy G.: Wind Shear and Turbulence Around Airports. A.R.A.P. Report No. 267, January 1976 .

33. Bradshaw, Peter: The Effects of Streamline Curvature on Turbulent Flow. AGARDograph No. 169, August 1973.

34. Bilanin, Alan $\mathrm{J}$. and Donaldson, Coleman duP.: Estimation of Velocities and Roll-Up in Aircraft Vortex Wakes. J. Aircraft, vol. 12, no. 7, July 1975, pp. 578-585.

35. Donaldson, Coleman duP.: The Relationship Between Eddy Transport and Second-Order Closure Models for Stratified Media and for Vortices. NASA SP-216, July 1972, pp. 233-255.

36. Barber, M.R., Hastings, Earl C., Jr., Champine, Robert A., and Tymczyszyn, Joseph J.: Vortex Attenuation Flight Experiments. Presented at NASA Vortex Wake Vortex Minimization Symposium, 25-26 February 1976. 